\title{
Theoretical Principles of Enhancer-Promoter Communication in
}

\section{Transcriptional Bursting}

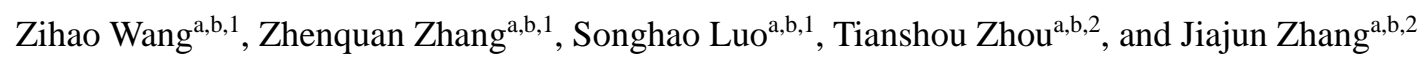

${ }^{a}$ Guangdong Province Key Laboratory of Computational Science School of Mathematics, Sun

Yat-sen University, Guangzhou 510275, P. R. China

${ }^{\mathrm{b}}$ School of Mathematics, Sun Yat-Sen University, Guangzhou 510275, P. R. China

${ }^{1}$ These authors contributed equally to this work.

${ }^{2}$ To whom correspondence may be addressed.

Email: mcszhtsh@mail.sysu.edu.cn or zhjiajun@mail.sysu.edu.cn

\begin{abstract}
Transcriptional regulation occurs through genomic contacts between enhancers and their cognate promoters, and most genes are transcribed in a bursty fashion. To understand the relationship between these two phenomena, we develop a general modeling framework in terms of the information transmission from upstream genomic organization to downstream transcriptional bursting. Importantly, we uncover fundamental theoretical principles of enhancer-promoter (E-P) spatial communication in the modulation of transcriptional burst size (BS) and burst frequency (BF). First, BS and BF obey their respective power-law dependences on the E-P communication strength and distinct scaling exponents. Second, the E-P spatial distance follows a Maxwell-Boltzmann distribution rather than the previously assumed Gauss distribution. Third, the E-P genomic distance affects transcriptional outcomes biphasically (i.e., in an exponential decay for small E-P genomic distances but insensitively to large E-P genomic distances). Fourth, the E-P communication mainly modulates BF rather than BS. Finally, the mutual information between BS (or BF) and E-P spatial distance further reveals essential characteristics of the information transfer from the upstream to the downstream. Our predictions are experimentally verifiable, e.g., confirmed by experimental data on Drosophila. The overall analysis provides insights into the role of the E-P communication in the control of transcriptional bursting.
\end{abstract}

\section{Significance}

Measurement technologies of chromatin conformations and genome-wide occupancy data of architectural proteins have revealed that genome topology is tightly intertwined with gene transcription. However, a long-standing question in transcriptional regulation is how the enhancerpromoter (E-P) spatial communication impacts transcriptional bursting kinetics. To address this issue, we develop a multiscale model that couples upstream chromatin dynamics to downstream transcriptional bursting. This model not only reveals fundamental principles of E-P communication in transcriptional bursting kinetics (e.g., burst size and frequency follow their own power-law behaviors) but also provides a general modeling framework toward the 4D nucleome project. 


\section{Introduction}

Gene transcription that is tightly related to three-dimensional (3D) genomic organization is a highly complex and regulated process that exhibits a discontinuous episodic bursting behavior (1-8) . As two cardinal regulatory elements, the promoter and the enhancer are responsible for the accurate spatiotemporal gene expression ensuring reliable cell functioning and cellular decision-making (914). Many experimental studies have been invested in understanding the roles of distal enhancers in regulating transcriptional bursting kinetics (15-19). However, the mechanism of how 3D chromatin organization (in particular 3D enhancer-promoter (E-P) communication) shapes transcriptional bursting patterns still remains elusive.

Hierarchic genomic structures captured by chromosome conformation capture and fluorescence in situ hybridization (FISH) as well as other experimental technologies have provided evidences for supporting various possible E-P topologies and linking upstream distinctive E-P communications to downstream gene transcription (20-28). Measurable sustained physical proximity of E-P communication, which is believed to increase the local concentrations of the coactivators and transcription factors (TFs), seems necessary for transcription in living Drosophila embryos $(18,29)$. This proximity is also needed for cells to execute correct gene expression programs, though the ways of E-P communication such as E-P loop and hub hypothesis are still a matter of debate (30, 31). To understand the mechanism of transcriptional bursting theoretically, many models of gene expression have been proposed, including simple models (e.g., the common ON-OFF model) that are mainly based on distinctive transient chromatin behaviors (32-37), and multistate models that seem a good candidate for mimicking complex promoter dynamics in mammalian cells $(8,19,38$ 45). However, these models ignored dynamic transcriptional regulation by spatial chromosome topology (46-50). To the best of our knowledge, a comprehensive theory of the information transmission from upstream chromatin organization to downstream transcriptional bursting is still lacking. And important yet fundamental questions such as how E-P communication shapes the observed patterns of mRNA expression and what is the role of the "range of action" for E-P proximity in the control of bursting kinetics remain unsolved. Understanding and revealing transcriptional bursting kinetics characterized by burst size (BS) and burst frequency (BF) require integrative models that consider both 3D chromatin motion (including E-P spatial communication) 
and upstream-to-downstream regulation.

A collection of experimental evidences has firmly established that the E-P communication strength can significantly raise transcriptional levels $(16,17,51,52)$, e.g., the sna distal shadow enhancer generates more bursts than the primary enhancer in Drosophila embryos (16). Recent liveimaging measurements have provided the clear evidence that E-P genomic distance can effectively control gene activities and thus affect transcriptional bursting kinetics $(16,53,54)$. These experimental observations indicate that the E-P genomic distance arranged by chromosomal rearrangements and the E-P communication strength limited by the E-P spatial distance as well as this distance itself are important parameters impacting the formation of transcriptional bursting patterns. Currently, a new trend is that genomic structures are stochastic at almost every level of organization and this stochasticity is suggestively linked to gene transcription and finally affects transcriptional outcomes $(26,27,46)$. Given the important impacts of these factors on transcriptional bursting, an unsolved and even theoretically unexplored issue is what principles govern the formation of transcriptional bursting patterns. How E-P spatial and genomic distances differently influence bursting patterns is unclear, either. As a matter of fact, biological systems are by nature multiscale. To date, many experimental studies have shown distinct timescale differences between upstream chromatin dynamics and downstream bursting kinetics $(55,56)$. For instance, E-P communication occurs on a timescale of seconds to minutes $(18,29,57)$, whereas half-lives of Pol II pause during transcription are on a timescale of minutes to hours (58-60). This temporal disconnection between the upstream and the downstream as well as the stochasticity of genomic organization and transcriptional bursting lies at the heart of a broad challenge in physical biology of forecasting transcriptional outcomes from the dynamics of underlying molecular processes.

Building upon experimental phenomena and acquired data, we develop a general theoretical framework to investigate how E-P communication shapes transcriptional bursting patterns, focusing on the uncovering of underlying molecular processes and dynamical mechanisms. This framework considers 3D upstream chromatin motion on a fast timescale and downstream mRNA bursty production on a slow timescale as well as the connection between the upstream and the downstream. We employ the classical differential Chapman-Kolmogorov Equation (dCKE, (61)) in stochastic process theory to analyze dynamic behaviors of transcriptional bursting across space and time, i.e., 4D transcriptional bursting kinetics $(62,63)$. Both model analysis and numerical simulations reveal 
fundamental principles of the E-P communication in modulating transcriptional bursting, e.g., BS and BF obey their own power laws, E-P spatial distance follows Maxwell-Boltzmann distribution instead of previously assumed Gauss distribution, and E-P communication mainly modulates BF but not BS. All these uncovered principles are verified by experimental results and seem universal. We emphasize that our model, which exhibits scalability by interpreting many experimental phenomena reported in the existing literatures $(16,17,51,53)$, provides a general modeling framework for investigating how chromatin dynamics affect transcriptional bursting kinetics in more realistic cases.

\section{Results}

\section{The First Principle Framework of Gene Transcriptional Bursting}

Transcription involving multiple regulatory elements is driven mainly by E-P communication (Fig. 1A), often exhibiting a burst-like pattern (Fig. 1B). Our goal is to develop an analysis framework that can reveal the essential mechanism of transcriptional bursting and predict possible dynamic behaviors compatible with experimental observations. This framework considers that upstream chromatin dynamics play on a fast timescale and downstream transcriptional bursting operates on a relatively slow timescale, but keeps the upstream and the downstream linked via a biologically reasonable way (Fig. 1D).

Our modeling framework is a general dCKE (61), whose form is as follows (see SI Appendix B1 for derivation)

$$
\frac{\partial \boldsymbol{p}(\boldsymbol{r}, \boldsymbol{s} ; t)}{\partial t}=-\nabla_{r} \cdot\left(\boldsymbol{p}(\boldsymbol{r}, \boldsymbol{s} ; t) \boldsymbol{V}(\boldsymbol{r}, \boldsymbol{s} ; t)^{\mathrm{T}}\right)+\nabla_{r}^{2}(\boldsymbol{D} \boldsymbol{p}(\boldsymbol{r}, \boldsymbol{s} ; t))+\boldsymbol{W}(\boldsymbol{r} ; t) \boldsymbol{p}(\boldsymbol{r}, \boldsymbol{s} ; t) .
$$

Here the column vector $\boldsymbol{p}(\boldsymbol{r}, \boldsymbol{s} ; t)$ represents the joint probabilities that the positions of $N$ nucleosomes are $r$ and the gene states are $s$ at time $t ; \nabla_{r}$ and $\nabla_{r}^{2}$ are the gradient operator and the Laplace operator respectively; $\boldsymbol{V}(\boldsymbol{r}, \boldsymbol{s} ; t)^{\mathrm{T}}$ represents the deterministic velocity field of chromatin dynamics with $\mathrm{T}$ standing for transpose; $\boldsymbol{D}$ is a diffusion matrix determining the additional noise strength of chromatin motion under isotropic diffusion with diffusion coefficient $D$; and $\boldsymbol{W}(\boldsymbol{r} ; t)$ is the nucleosome position-dependent transition matrix for all gene states. The first term on the right-hand side of Eq. [1] represents a deterministic component modeling upstream 
B

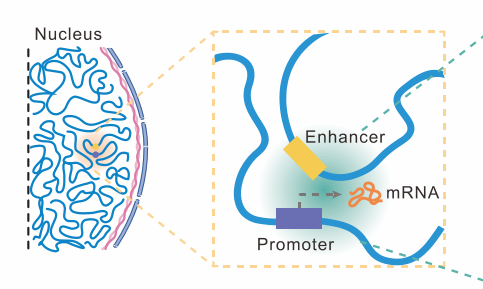

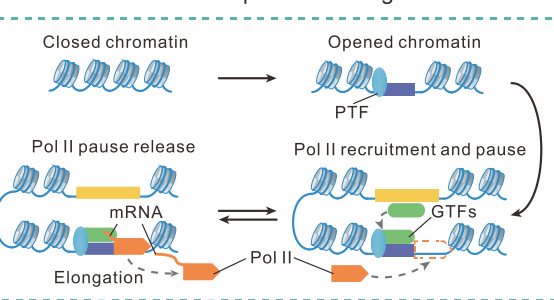

C

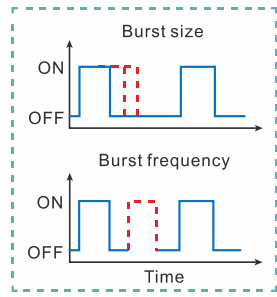

$\mathrm{D}$

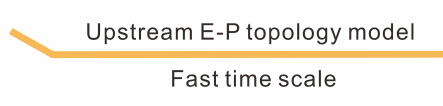

$\mathrm{E}$

$\mathrm{F}$

Regulating Pattern

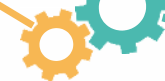

Downstream transcriptional bursting model

Slow time scale

G
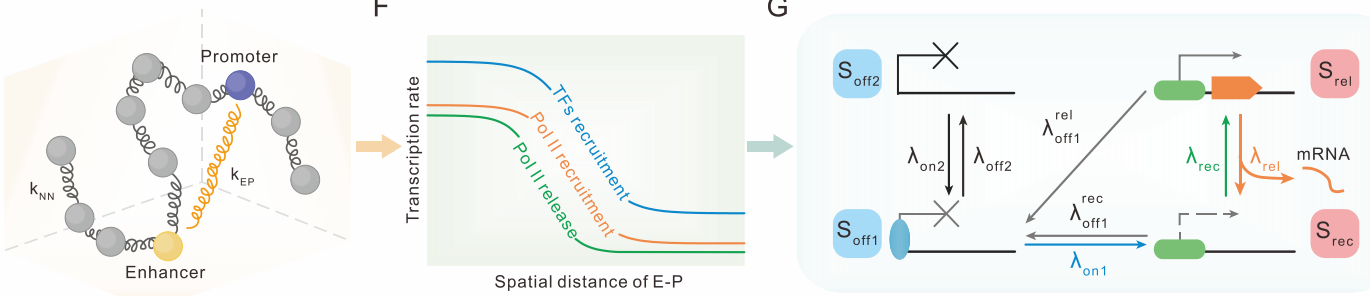

Spatial distance of E-P

Fig. 1. A framework for studying how upstream E-P communication shapes downstream mRNA bursting patterns. (A) E-P communication plays a key role in regulating transcriptional bursting in cell nucleus. $(B)$ The downstream transcription cycle is a multistep process guided by upstream E-P communication, where only the main steps are depicted. (C) Burst size and burst frequency as two quantitative indices to measure the entire bursting system. (D) An information flow viewpoint for bridging the temporal disconnection between upstream E-P topology and downstream transcriptional bursting. $(E)$ A generalized Rouse model with additional E-P communication (indicated by the yellow spring with coefficient $k_{\mathrm{EP}}$ ) for simulating chromatin dynamics. $(F)$ A schematic for input-output functions, where the upstream E-P spatial distance is taken as input whereas gene state switching rates as outputs. $(G)$ A four-state model for imitating transcriptional bursting. Here $\lambda_{\mathrm{on} 2}$ and $\lambda_{\mathrm{off} 2}$ are switching rates between a deep inactive state $\left(\mathrm{S}_{\mathrm{off2}}\right)$ and a primed but inactive state $\left(\mathrm{S}_{\mathrm{off} 1}\right) ; \lambda_{\text {offl }}^{\text {rel }}$ is a transition rate from $\mathrm{S}_{\text {rel }}$ (representing Pol II pause release state) to $\mathrm{S}_{\text {off } 1} ; \lambda_{\text {offl }}^{\text {rec }}$ and $\lambda_{\text {on1 }}$ are transition rates from $S_{\text {rec }}$ (representing Pol II recruitment state) to $S_{\text {offl }}$ and from $S_{\text {offl }}$ to $S_{\text {rec }}$, respectively; $\lambda_{\text {rel }}$ and $\lambda_{\text {rec }}$ are switching rates between $S_{\text {rec }}$ and $S_{\text {rel }}$.

chromatin motion and the second term represents a stochastic component accounting for random fluctuations, both altogether describing the upstream chromatin's spatiotemporal diffusion process. The last term captures downstream gene states' randomly switching process driven by molecular 
events such as chromatin remodeling, TFs binding, and Pol II cluster. We point out that Eq. [1] is the first theoretical model that comprehensively considers upstream and downstream molecular processes and the connection between the upstream and the downstream, and can be taken as a good starting point for analyzing how chromatin motion affects transcriptional bursting in complex cases.

From a physical viewpoint, upstream chromatin can be modeled as a polymer discretized into a collection of successive monomers connected by harmonic spring (64-67). Then, chromatin motion evolves according to the overdamped equation $\mathrm{d} \boldsymbol{r}=\boldsymbol{V}(\boldsymbol{r}, \boldsymbol{s} ; t) \mathrm{d} t+\sqrt{2 D} \mathrm{~d} \boldsymbol{B}(t)$, where $\boldsymbol{B}(t)$ is a vector of independent Brownian motions (see SI Appendix C1). We assume that changes in gene state do not affect chromatin motion although transcriptional outcomes may impact chromatin configurations $(68,69)$. Therefore, $\boldsymbol{V}(\boldsymbol{r}, \boldsymbol{s} ; t)$ can be approximated as $\boldsymbol{V}(\boldsymbol{r} ; t)=-\nabla_{\boldsymbol{r}} U(\boldsymbol{r} ; t) / \gamma$, where $U(\boldsymbol{r} ; t)$ is the total potential of chromatin conformation and $\gamma$ is a friction coefficient. Note that $U(\boldsymbol{r} ; t)$ can be decomposed as $U(\boldsymbol{r} ; t)=U_{\mathrm{NN}}(\boldsymbol{r} ; t)+U_{\mathrm{EP}}(\boldsymbol{r} ; t)$, where the potential $U_{\mathrm{NN}}(\boldsymbol{r} ; t)$ for the chain connection of $N$ monomers is set as $(1 / 2) \sum_{j=1}^{N-1} k_{\mathrm{NN}}\left(\boldsymbol{r}_{j}-\boldsymbol{r}_{j+1}\right)^{2}$ with $k_{\mathrm{NN}}$ being the spring coefficient and the potential $U_{\mathrm{EP}}(\boldsymbol{r} ; t)$ for the E-P communication is set as $(1 / 2) k_{\mathrm{EP}}\left(\boldsymbol{r}_{\mathrm{E}}-\boldsymbol{r}_{\mathrm{P}}\right)^{2}$ with $\mathrm{E}, \mathrm{P} \in\{1, \cdots, N\}$ representing the enhancer and the promoter respectively. Here $k_{\mathrm{EP}}$ represents the E-P communication strength and will be taken as a key parameter of our model (Fig. 1E). It should be pointed out that the above model is simplified since realistic molecular processes underlying chromatin motion would be complex, e.g., multiple promoters may interact with one enhancer (70), and chromatin's spatiotemporal diffusion may be anomalous (71, 72). However, the fact that model predictions are in good agreement with experimental measurements indicates the reasonability of our model assumptions (SI Appendix, Fig. S10B).

Downstream transcription apparatus can be characterized by promoter-state switching (Fig. 1B). In line with previous propositions $(52,73)$, we introduce a four-state regulatory architecture with each state transition corresponding to a biochemical process (Fig. 1G, see SI Appendix C2). Specifically, a deep inactive state $\left(\mathrm{S}_{\text {off } 2}\right)$ and a primed but inactive state $\left(\mathrm{S}_{\text {off } 1}\right)$, integrated as the OFF state, are used to explain chromatin remodeling and TF binding. Pol II recruitment state $\left(\mathrm{S}_{\text {rec }}\right.$, (74)) and Pol II pause release state $\left(S_{\text {rel }},(75)\right)$, two critical processes involved in transcriptional 
bursting, are taken as the ON state, and mRNA generation is accompanied by the transition from $\mathrm{S}_{\text {rel }}$ to $\mathrm{S}_{\mathrm{rec}}$. If states $\mathrm{S}_{\mathrm{rel}}$ and $\mathrm{S}_{\mathrm{rec}}$ switch multiple times during one ON period, transcription will occur in a burst-like manner. Switching rates between these states are depicted in Fig. 1G and the elements of state transition matrix $\boldsymbol{W}$ in Eq. [1] are related to gene state switching rates (see $S I$ Appendix C2).

After identifying the above formulations and settings, we build a link for the information flow from upstream chromatin conformations to downstream biochemical reactions (Fig. 1F). E-P communication carries the upstream information to orchestrate bursting patterns, and downstream transition rates change accordingly. We introduce input-output functions in which E-P spatial distance $d_{\mathrm{S}}$ is taken as input and $d_{\mathrm{S}}$-dependent gene state switching rates are treated as outputs, thus bridging the upstream and the downstream (see SI Appendix C3). Although how E-P communication is implemented is arguing $(30,31)$, here we adopt a hub hypothesis that each of switching rates $\lambda_{\text {on } 1}, \lambda_{\text {rec }}$ and $\lambda_{\text {rel }}$ is a piecewise Hill-like function of $d_{\mathrm{s}}$, i.e. $\lambda_{c}\left(d_{\mathrm{S}}\right)$, $c \in\{$ on1,rec,rel $\}$ (Fig. 1F). This assumption would be idealistic compared to elaborate organisms, but all the above settings constitute an analysis framework that can be used in the study of complex transcriptional regulation.

It is worth mentioning that we have developed both an effective analytical approach and a numerical simulation algorithm for solving the above entire system (SI Appendix B2 gives details of the algorithm implementation and SI Appendix E presents analytical details). These methods provide a systematic approach for tracing the respective contributions of the system's key parameters (e.g., E-P communication strength, E-P genomic distances) to the experimentally observable patterns of transcriptional bursting and further for revealing the essential mechanism of bursting kinetics.

\section{Distribution characteristics of transcriptional bursting}

BS (defined as the number of mRNA molecules produced per burst) and BF (defined as the number of bursts occurred per unit time) are two important quantities characterizing bursting kinetics but are also two random variables (Fig. 1C). In order to reveal the characteristics of BS and BF, we try to find the BS distribution $p_{B S}(m)$ and the cycle time (CT) distribution $p_{C T}(t)$, where CT is 
defined as the total time that the gene dwells at OFF and ON states, and BF as the reciprocal of CT. In the following, $p_{B S}(m)$ and $p_{C T}(t)$ are denoted uniformly by $p_{X}(x)$, where $X \in\{B S, C T\}$.

Note that the entire transcriptional system involves two kinds of timescales - the fast motion of upstream chromatin conformation and the slow occurrence of downstream transcription reactions. To derive distribution $p_{X}(x)$ directly from this multiscale system is a particularly challenge task. Therefore, we resort to a timescale separation method that corresponds to the two limits in which the upstream chromatin motion is either much faster or much slower than the downstream transcription reactions. This method has been successfully used in the analysis of gene regulatory networks $(76,77)$ and the mutual information that disentangles interactions from changing environments (78).

Specifically, we introduce a scaling parameter $\omega$, defined as the ratio of the minimum variable transition rate and the maximum chromatin motion velocity, to represent the maximum achievable ratio of upstream and downstream timescales. If the timescale of the upstream motion is much faster than that of the downstream reactions $(\omega \ll 1)$, the quasi-stationary upstream can be well separated from the downstream transcription. Then, $p_{X}(x)$ can be analytically calculated through $p_{X}^{\text {Fast }}(x)=p_{X|D S\rangle}\left(x \mid \int_{0}^{+\infty} \lambda_{c}\left(d_{\mathrm{S}}\right) p_{D S}\left(d_{\mathrm{S}}\right) \mathrm{d} d_{\mathrm{S}}\right)$ (SI Appendix, Fig. S12C), where $p_{D S}\left(d_{\mathrm{S}}\right)$ is upstream E-P spatial distance distribution and $\langle D S\rangle$ is the expectation of E-P spatial distance. Oppositely, if the timescale of the upstream motion is much slower than that of the downstream reactions ( $\omega \gg 1$ ), then according to total probability principle, $p_{X}(x)$ can be calculated through $p_{X}^{\text {Slow }}(x)=\int_{0}^{+\infty} p_{X \mid D S}\left(x \mid \lambda_{c}\left(d_{\mathrm{S}}\right)\right) p_{D S}\left(d_{\mathrm{S}}\right) \mathrm{d} d_{\mathrm{S}}$ (SI Appendix, Fig. S12E). For the intermediate range of $\omega$ where there is no timescale separation, we find that $p_{X}(x)$ can well fit with the following interpolation formula (again see SI Appendix E1)

$$
p_{X}(x)=\frac{1}{1+\omega} p_{X}^{\text {Fast }}(x)+\frac{\omega}{1+\omega} p_{X}^{\text {Slow }}(x) .
$$

It should be pointed out that this interpolation formula needs to know E-P spatial distance distribution $p_{D S}\left(d_{\mathrm{S}}\right)$ and conditional distribution $p_{X \mid D S}\left(x \mid \lambda_{c}\left(d_{\mathrm{S}}\right)\right)$ given E-P spatial distance $d_{\mathrm{S}}$. By complex calculations, we find that E-P spatial distance $d_{\mathrm{S}}$ obeys the following exact 
Maxwell-Boltzmann distribution (see SI Appendix E2, Fig. S10A) but not previously assumed Gauss distribution (18)

$$
p_{D S}\left(d_{\mathrm{S}}\right)=\sqrt{\frac{2}{\pi}} \Theta^{-3} d_{\mathrm{S}}^{2} \exp \left(-\frac{d_{\mathrm{S}}^{2}}{2 \Theta^{2}}\right)
$$

where $\Theta=\sqrt{D \gamma\left(k_{\mathrm{NN}} / d_{\mathrm{G}}+k_{\mathrm{EP}}\right)^{-1}}$, a compound parameter depending on the genomic property, can determine how changes to any of the concerned parameters such as E-P communication strength $k_{\mathrm{EP}}$ or E-P genomic distance $d_{\mathrm{G}}$ (different from E-P spatial distance $d_{\mathrm{S}}$ ) alter the shape of the $d_{\mathrm{S}}$ distribution (SI Appendix, Fig. S10C). Note that the sum $k_{\mathrm{NN}} / d_{\mathrm{G}}+k_{\mathrm{EP}}$ can represent the integrative effect of two paralleling springs, possibly hinting the principle of engineering communication between regulatory elements. Quantitative measurements of the distance between Sox 2 and its essential enhancer in living mouse embryonic stem cells have indicated the validity of this Maxwell-Boltzmann distribution (see SI Appendix J, Fig. S10B, (79)). It is worth pointing out that Eq. [3] also uncovers a power-law behavior between encounter probabilities and E-P spatial (or genomic) distance, which is in accordance with experimental data for consecutive and nonconsecutive TAD borders in Drosophila (see SI Appendix J, Fig. S10E, (80)). In addition, the combination of $k_{\mathrm{NN}}$ equaling 0.1 and $d_{\mathrm{G}}$ taking 50 shows that the mean E-P spatial distance is also consistent with that of experimental data (see SI Appendix J).

Note that $p_{X \mid D S}\left(x \mid \lambda_{c}\left(d_{\mathrm{S}}\right)\right)$ is merely related to given transition rates and can be then given analytically (see SI Appendix E3). Furthermore, we can analytically show that the BS follows a geometric distribution $\operatorname{Geo}(\theta)$ where parameter $\theta$ represents the success probability of burst termination; the duration of OFF period follows a bi-exponential distribution, supporting that gene inactivation to activation is not necessarily a one-step process following an exponential distribution $(8,43,81,82)$; and the CT distribution is expressed as the weighted combination of multiple exponential distributions, which demonstrates a non-origin peak (SI Appendix, Fig. S11).

Finally in this subsection, we point out that Eq. [2] provides high-accuracy approximations of downstream transcriptional outcomes (SI Appendix, Fig. S13) and is a useful and simple formula for predicting the dynamics of mRNA over a broad range of timescale separation. By the combination of Eq. [2] and Eq. [3], we can further forecast how upstream chromatin random organization impacts downstream transcriptional outcomes. 


\section{Transcriptional bursting kinetics follow power laws}

With the above arsenal of theoretical analysis, we further explore how E-P communication strength $k_{\mathrm{EP}}$ qualitatively affects transcriptional bursting kinetics (mainly, BS and BF). As one of the most common modular organisms, Drosophila has been extensively studied in terms of bursting $(83,84)$. In our analysis, we take the inherent communication strength between distinctive enhancers such as sna shadow and sna primary enhancer in Drosophila $(16,85,86)$ as the value of $k_{\mathrm{EP}}$, but make use of the fact that effective experimental means such as external hormone (87) or heavy metal stimulations (88) can alter $k_{\mathrm{EP}}$. In order to reveal the distinct effects of different $k_{\mathrm{EP}}$ on bursting kinetics, we keep the E-P genomic distance fixed (Fig. 2A).

A

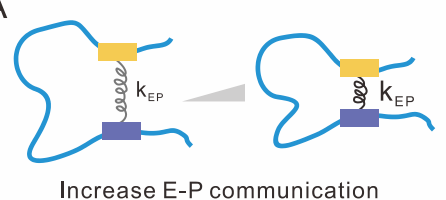

B
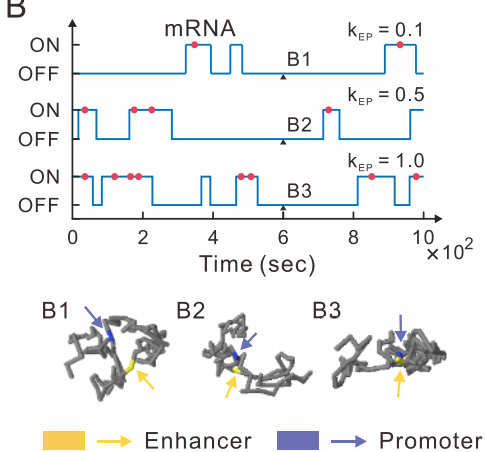

C

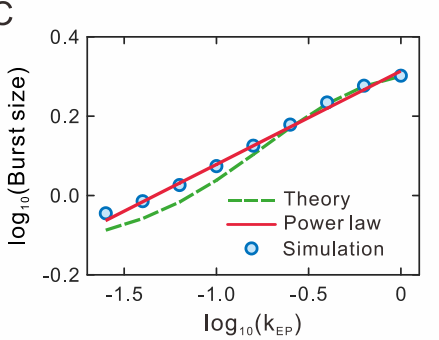

E

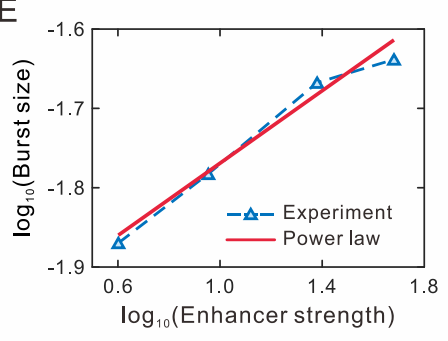

$\mathrm{D}$

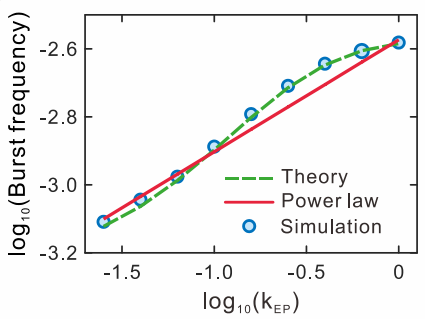

$\mathrm{F}$

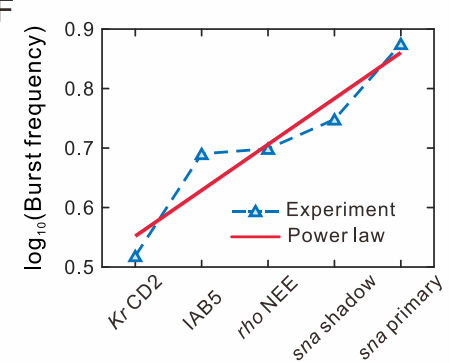

Fig. 2. Effect of the E-P communication strength on transcriptional bursting kinetics. $(A)$

Schematic illustration of the augment of E-P communication strength $k_{\mathrm{EP}} \cdot(B)$ Example traces of binarized transcriptional activity for three different values of $k_{\mathrm{EP}}$, where red points represent the mRNA generation events and the corresponding 3D chromatin structures are shown on the bottom. (C) Log-log plot for the relationship between mean burst size and strength $k_{\mathrm{EP}}$. The green dashed line represents the theoretical analysis result (Eq. [2]), whereas blue circles show numerical simulations. The red solid line indicates a power-law approximation (Eq. [4]) with scaling exponent $S_{\mathrm{BS}}=0.23$. (D) Log-log plot for the relationship between burst frequency and $k_{\mathrm{EP}}$. Meanings of symbols are the same as those in $(C)$, but the scaling exponent is $S_{\mathrm{BF}}=0.33$. (E) Log-log plot of experimental data from (17). (F) Plot of experimental data from (16). The labels of the $x$-axis represent the sna shadow, sna primary, rho NEE, IAB5, and $K r \mathrm{CD} 2$ enhancers, respectively. 
Fig. 2B illustrates how changes in $k_{\mathrm{EP}}$ alter chromatin conformations and further affect bursting profiles. Theoretically, a larger (smaller) $k_{\mathrm{EP}}$ corresponds to a shorter (longer) E-P spatial distance, and the increasing $k_{\mathrm{EP}}$ can boost both BS and BF (SI Appendix, Figs. S10C, S13A-B). These features have been qualitatively supported by many experimental evidences from different organisms $(16,17,52,89)$. Notably, since ON state dwell-time remains fundamentally the same, the amplified BF can be achieved by progressively reducing OFF state dwell-time (SI Appendix, Figs. S13C-D, $(19,89,90))$. This implies that the gene state switching rate $\lambda_{\text {on1 }}$, associated with TF recruitment in biology, is a principal parameter affecting $\mathrm{BF}$, in agreement with the experimental finding that a higher TF level leads to a higher BF on the c-Fos gene (51).

To reveal the qualitative relevance of bursting kinetics to E-P communication strength, we further analyze two logarithmic gains $\partial \log _{10} \mathrm{BS} / \partial \log _{10} k_{\mathrm{EP}}$ and $\partial \log _{10} \mathrm{BF} / \partial \log _{10} k_{\mathrm{EP}}$, which measure how $\mathrm{BS}$ and $\mathrm{BF}$ are affected by $k_{\mathrm{EP}}$. Binary approximations of gene state switching rates provide a good strategy for simplifying analysis including estimating BS and BF (see SI Appendix F1, Figs. S14D-E). Our theoretical analysis shows that the logarithmic gains are about zero when $k_{\mathrm{EP}}$ is too small $\left(k_{\mathrm{EP}}<0.01\right)$ or too large $\left(k_{\mathrm{EP}}>1\right)$ (SI Appendix, Figs. S14A-C), implying that transcriptional burst response is insensitive to $k_{\mathrm{EP}}$ in this case. Then we focus on a reasonable biological range of $k_{\mathrm{EP}}\left(0.01<k_{\mathrm{EP}}<1\right)$. The logarithmic gains are almost constant and $\mathrm{BS}$ and $\mathrm{BF}$ can be thus approximated with linear functions of $k_{\mathrm{EP}}$ in logarithmic scale (Fig. 2C-D, SI Appendix, Figs. S14D-E). Concretely, BS and BF approximately obey the following power-law behaviors

$$
\text { burst size } \sim\left(k_{\mathrm{EP}}\right)^{S_{\mathrm{BS}}} \text {, burst frequency } \sim\left(k_{\mathrm{EP}}\right)^{S_{\mathrm{BF}}} \text {, }
$$

where $S_{\mathrm{BS}}$ and $S_{\mathrm{BF}}$ are two positive scaling exponents that can be theoretically estimated (see $S I$ Appendix F2). Note that these two different scaling exponents, e.g., $S_{\mathrm{BS}}=0.23$ in Fig. $2 \mathrm{C}$ and $S_{\mathrm{BF}}=0.30$ in Fig. 2D, are critical indices since they reflect the ability of the BS and BF responses to E-P communication, which will be in details analyzed in the next section. To ascertain whether the power-law behaviors shown in Eq. [4] always hold, we let key parameters change in broad ranges. For all the possible cases of parameter values, we find that log-log plots between BS (or BF) and $k_{\mathrm{EP}}$ still demonstrate an approximately linear relationship, implying that the growing tendency can be featured by power-law relationships (SI Appendix, Fig. S15).

The above theoretical predictions have been verified by experimental data. For example, the 
addition of estradiol that triggers stronger contacts between $\beta$-globin and its locus control region over time leads to the increase of $\beta$-globin gene transcriptional BS (17). If we use the erythroid maturation time after adding the hormone to reflect the E-P communication strength $k_{\mathrm{EP}}$, the BS of $\beta$-globin gene can well fit with a power-law function (Fig. 2E). In another example, transgene experiments examining the effects of differential enhancers on BF showed that a stronger enhancer such as sna shadow produces more bursts than a weak enhancer such as rho NEE in living Drosophila embryos (16). We find that the corresponding BF exhibits a power-law-like behavior if the enhancer's strength increases in an equidistant interval on a logarithmic scale (Fig. 2F). SI Appendix Figs. S16A-C demonstrate more experimental data $(16,51)$ on the dependences of BS and $\mathrm{BF}$ as well as the mRNA expression level (approximately equals to the product of $\mathrm{BS}$ and the $\mathrm{BF}$ (91)) on E-P communication strengths, further verifying these power-law behaviors.

\section{E-P communication mainly modulates burst frequency rather than burst size}

Eq. [4] indicates that BS and BF regulated by E-P communication strength $k_{\mathrm{EP}}$ follow their own power-law behaviors. Since $S_{\mathrm{BS}}$ and $S_{\mathrm{BF}}$ in Eq. [4] reflect the regulation ability of $k_{\mathrm{EP}}$, we next compare the sizes of $S_{\mathrm{BS}}$ and $S_{\mathrm{BF}}$, i.e., compute the ratio of $S_{\mathrm{BF}} / S_{\mathrm{BS}}$, to show which of BS and $\mathrm{BF}$ is primarily regulated by E-P communication. For this goal, we let E-P communication strength and all gene state switching rates change in broad ranges. In general, extracting insights from directly analyzing the effects of these changes in the high-dimensional parameter space consisting of E-P communication strength and all gene state switching rates on BS and BF is very difficult. Therefore, we resort to a dimension reduction method that maps the high dimensional parameter space into an experimentally measurable and theoretically computable two-dimensional space (Fig. 3A) in which two indices $\rho_{\mathrm{BS}}$ and $\rho_{\mathrm{BF}}$ are defined as the ratios of the maximum $\mathrm{BS}$ and BF over the minimum $\mathrm{BS}$ and $\mathrm{BF}$, respectively.

Next, we seek to calculate the value of $S_{\mathrm{BF}} / S_{\mathrm{BS}}$ in the $\left(\rho_{\mathrm{BF}}, \rho_{\mathrm{BS}}\right)$ space. Note that one point in the low dimensional space possibly corresponds to multiple points in the high-dimensional space due to the irreversibility of the mapping. To overcome this multiple-to-one difficulty, We use the averaging method to estimate ratio $S_{\mathrm{BF}} / S_{\mathrm{BS}}$, finding that this ratio generally increases with the growth of $\rho_{\mathrm{BF}}$ or with the shrinking of $\rho_{\mathrm{BS}}$ (Fig. 3A, SI Appendix, Fig. S17A). In Fig. 3B, the separatrix $S_{\mathrm{BF}} / S_{\mathrm{BS}}=1$ (red line) separates the space into two regions: upper $S_{\mathrm{BF}}>S_{\mathrm{BS}}$ and lower 

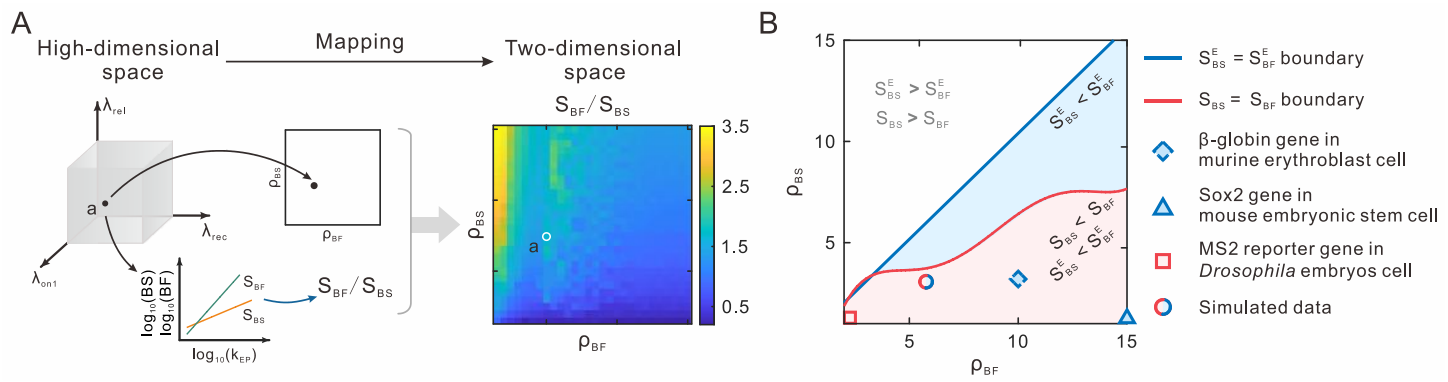

Fig. 3. Separatrixes for power-law behaviors of $\mathrm{BS}$ and $\mathrm{BF}$ in the $\left(\rho_{\mathrm{BF}}, \rho_{\mathrm{BS}}\right)$ plane. $(A)$

Schematic illustration of the dimension reduction method, where a high-dimensional parameter space is mapped into the $\left(\rho_{\mathrm{BF}}, \rho_{\mathrm{BS}}\right)$ space (from the parameter coordinate space to the square) and the calculated values of $S_{\mathrm{BF}} / S_{\mathrm{BS}}$ (the bottom inset) are plotted in the $\left(\rho_{\mathrm{BF}}, \rho_{\mathrm{BS}}\right)$ plane (the heatmap). (B) The red line stands for the boundary obtained by smoothing the theoretical analysis, the blue line for the boundary after enhancer deletion. The colored regions indicate that E-P communication affects BF more than BS under different situations. The square at point (2.3,1.3) corresponds to the data in $(16)$, the triangle at point $(15,1.4)$ to the data in $(92)$, the dashed diamond at point $(\cdot, 3.3)$ to the data in (17), and the red blue mixed circle to the simulated data in this paper. Note that the BF in (92) defined as the reciprocal of OFF state dwell-time, which is approximately converted to the reciprocal of $\mathrm{CT}$, and that the $\rho_{\mathrm{BF}}$ at the dashed diamond point related to burst fraction in (17) is approximate. By the blue region, we mean the whole areas below the blue line (some part is covered by red regions).

$S_{\mathrm{BF}}<S_{\mathrm{BS}}$. In particular, the data shown in Figs. 2C and 2D correspond to the point $\left(\rho_{\mathrm{BF}}, \rho_{\mathrm{BS}}\right)=(5.8,3.1)$, which apparently locates in the area of $S_{\mathrm{BS}}<S_{\mathrm{BF}} \quad\left(S_{\mathrm{BF}} / S_{\mathrm{BS}}=1.4\right.$, Fig. 3B, red-blue mixed circle). To show the reliability of this averaging method, we use the minimum (or maximum) value rather than the mean to compute $S_{\mathrm{BF}} / S_{\mathrm{BS}}$. As a result, the separatrix $S_{\mathrm{BF}} / S_{\mathrm{BS}}=1$ displays a similar change trend, i.e., the range of the $\rho_{\mathrm{BS}}$ satisfying $S_{\mathrm{BS}}<S_{\mathrm{BF}}$ gradually becomes larger with the increase of $\rho_{\mathrm{BF}}$ (SI Appendix, Figs. S17A-B).

To complete the above analysis, we consider enhancer deletion where $k_{\mathrm{EP}}$ is exactly zero but out of the range $0.01<k_{\mathrm{EP}}<1$ (SI Appendix, Fig. S11A). For the same parameter values as in the above calculation, we find that the sizes of $\rho_{\mathrm{BS}}$ and $\rho_{\mathrm{BF}}$ with enhancer deletion are the same as those with enhancer regulation but the slope ratio $S_{\mathrm{BF}}^{\mathrm{E}} / S_{\mathrm{BS}}^{\mathrm{E}}(=1.56)$ (representing the ratio after enhancer deletion, see SI Appendix $\mathrm{G}$ ) is different from the value of $S_{\mathrm{BF}} / S_{\mathrm{BS}}(=1.4)$ (Fig. 3B, red- 
blue mixed circle). Using the results obtained by a similar method of calculating $S_{\mathrm{BF}} / S_{\mathrm{BS}}$, we draw the separatrix $S_{\mathrm{BF}}^{\mathrm{E}} / S_{\mathrm{BS}}^{\mathrm{E}}=1$ in Fig. 3B (blue line). Then we find that no matter whether enhancer deletion is considered, this does not influence $\rho_{\mathrm{BS}}$ and $\rho_{\mathrm{BF}}$, but impacts ratios $S_{\mathrm{BF}} / S_{\mathrm{BS}}$ and $S_{\mathrm{BF}}^{\mathrm{E}} / S_{\mathrm{BS}}^{\mathrm{E}} \quad$ (referring to two different oblique lines in SI Appendix, Fig. S11F).

Finally, we locate the experimental data into the $\left(\rho_{\mathrm{BF}}, \rho_{\mathrm{BS}}\right)$ space and determine which of BS and $\mathrm{BF}$ is mainly regulated by E-P communication. Although experimental data on gene state switching rates lack, we may use the maximum and minimum BS and BF experimentally available to compute $\rho_{\mathrm{BS}}$ and $\rho_{\mathrm{BF}}$. Based on analysis of data from transgene experiments, we find that the MS2 reporter gene displays different bursting kinetics for different enhancer strengths, e.g., for a stronger sna shadow enhancer and a weaker $K r$ CD2 enhancer, the result is $\left(\rho_{\mathrm{BF}}, \rho_{\mathrm{BS}}\right)=(2.3,1.3)$ (Fig. 3B, red square point, (16)). The maximum BS of the gene $\beta$-globin measured in an experiment of erythroid maturation is over three times the minimum gained by the deletion of the LCR enhancer (Fig. 3B, dashed blue diamond point, (17)). In addition, based on the Sox 2 gene expression data with and without enhancer deletion in normal ES cells, we can infer that the BS reduces 1.4 times and the BF reduces roughly 15 times (Fig. 3B, blue triangle point, (92)). Interestingly, all these analyzed experimental data are all located in a narrower region of $S_{\mathrm{BS}}<S_{\mathrm{BF}}$ (the right lower region in Fig. 3B), further verifying that the E-P communication mainly modulates BF rather than BS.

\section{Threshold phenomena in modulation of bursting kinetics by E-P genomic distance}

Having devled into the qualitative effect of E-P communication strength $k_{\mathrm{EP}}$ on bursting kinetics as described in Eq. [4], we next analyze how E-P genomic distance $d_{\mathrm{G}}$ quantitatively impacts transcriptional activities and further bursting kinetics. To focus on the effect of $d_{\mathrm{G}}$ on BS and BF, we freeze $k_{\mathrm{EP}}$ but let $d_{\mathrm{G}}$ increase. This can be achieved by arranging the enhancer and the promoter symmetrically on the chain and letting them move in the opposite direction along the chain (Fig. 4A).

From Eq. [3], we see that $d_{\mathrm{G}}$ and $k_{\mathrm{EP}}$ have the just opposite impact on E-P spatial distance (SI Appendix, Fig. S10C). By the similar method of analyzing the effect of $k_{\mathrm{EP}}$ on BS and BF, we find that two logarithmic gains $\partial \log _{10} \mathrm{BS} / \partial \log _{10} d_{\mathrm{G}}$ and $\partial \log _{10} \mathrm{BF} / \partial \log _{10} d_{\mathrm{G}}$ are negative, implying that the increasing E-P genomic distance can reduce BS and BF (SI Appendix, Figs. S14F- 
H). With the further increase of $d_{\mathrm{G}}$, both BS and BF finally reduce to constants. These analyses indicate that the responses of BS and BF to the E-P genomic distance exhibit biphasic (first descent and then stable) change tendencies if other system parameters are fixed (Figs. 4B and 4C).

In order to confirm the above biphasic behaviors obtained by theoretical analysis, we first carry out numerical simulations and then perform the Kruskal-Wallis nonparametric test for simulated samples. This statistical test can identify the turning point of the biphasic responses of BS and BF to $d_{\mathrm{G}}$. Here the turning point (referring to black dashed lines in Figs. $4 \mathrm{~B}$ and $4 \mathrm{C}$ ) is defined as the value of $d_{\mathrm{G}}$ for which the Kruskal-Wallis test has no significant difference for the first time (referring to grey circles in Figs. $4 \mathrm{~B}$ and $4 \mathrm{C}$ ). We denote by $d_{\mathrm{G}}^{\mathrm{BS}}$ and $d_{\mathrm{G}}^{\mathrm{BF}}$ the $d_{\mathrm{G}}$ values corresponding to the turning points of BS and BF respectively. Then, we observe from Figs. 4B and $4 \mathrm{C}$ that $d_{\mathrm{G}}^{\mathrm{BS}}$ is smaller than $d_{\mathrm{G}}^{\mathrm{BF}}$. This difference highlights the importance of BF in the E-P communication-based regulation of transcriptional bursting kinetics.

Furthermore, we seek to use functions to characterize the above biphasic behaviors. Note that the logarithmic gains of $\mathrm{BS}$ and $\mathrm{BF}$ are almost constant for $d_{\mathrm{G}}$ in the first phase (i.e., $d_{\mathrm{G}} \leq d_{\mathrm{G}}^{\mathrm{BS}}$ or $d_{\mathrm{G}} \leq d_{\mathrm{G}}^{\mathrm{BF}}$ ), implying that in this phase, the logarithmic gains can be approximated as linear functions of $d_{\mathrm{G}}$ or equivalently, BS and BF can be approximated as power functions of $d_{\mathrm{G}}$ with exponents $S_{\mathrm{BS}}$ and $S_{\mathrm{BF}}$ respectively (see SI Appendix F2, Figs. S14I-J). For the second phase (i.e., $d_{\mathrm{G}}>d_{\mathrm{G}}^{\mathrm{BS}}$ or $\left.d_{\mathrm{G}}>d_{\mathrm{G}}^{\mathrm{BF}}\right), \mathrm{BS}$ and $\mathrm{BF}$ are all constants. In summary, the change tendencies of BS and BF can be mathematically described as

$$
\text { burst size } \sim\left\{\begin{array} { l l } 
{ ( d _ { \mathrm { G } } ) ^ { S _ { \mathrm { BS } } } , } & { d _ { \mathrm { G } } \leq d _ { \mathrm { G } } ^ { \mathrm { BS } } , } \\
{ \text { const. } , } & { d _ { \mathrm { G } } > d _ { \mathrm { G } } ^ { \mathrm { BS } } , }
\end{array} \text { burst frequency } \sim \left\{\begin{array}{cc}
\left(d_{\mathrm{G}}\right)^{S_{\mathrm{BF}}}, & d_{\mathrm{G}} \leq d_{\mathrm{G}}^{\mathrm{BF}}, \\
\text { const. }, & d_{\mathrm{G}}>d_{\mathrm{G}}^{\mathrm{BF}} .
\end{array}\right.\right.
$$

Here $S_{\mathrm{BS}}$ and $S_{\mathrm{BF}}$ are two negative scaling exponents that can be theoretically estimated. Note that these scaling exponents are in general different (e.g., $S_{\mathrm{BS}}=-0.16$ in Fig. 4D, red) and BF (e.g., $S_{\mathrm{BF}}=-0.19$ in Fig. 4E, red). An intuitive explanation for the asymptotic trend in large $d_{\mathrm{G}}$ is that the lumping parameter $\Theta=\sqrt{D \gamma\left(k_{\mathrm{NN}} / d_{\mathrm{G}}+k_{\mathrm{EP}}\right)^{-1}}$ in Eq. [3] approaches to a constant with the enlargement of $d_{\mathrm{G}}$, indicating that for sufficiently large $d_{\mathrm{G}}$, the effect of E-P communication on transcriptional bursting is mainly determined by communication strength $k_{\mathrm{EP}}$ rather than by E-P genomic distance $d_{\mathrm{G}}$. 
A

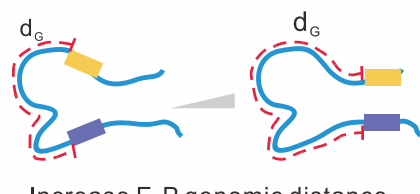

Increase E-P genomic distance

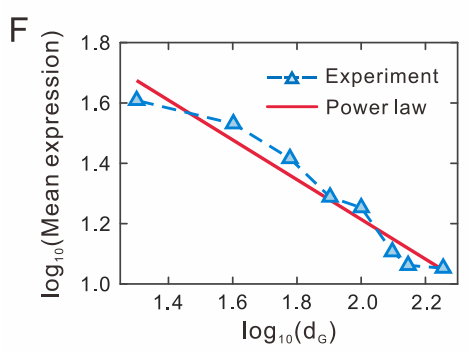

B

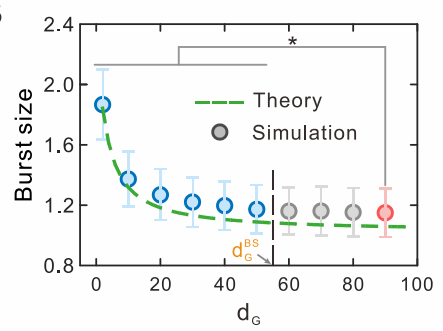

$\mathrm{D}$

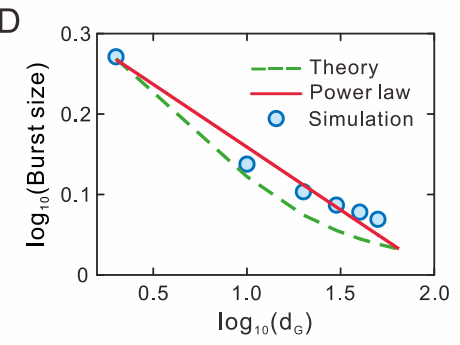

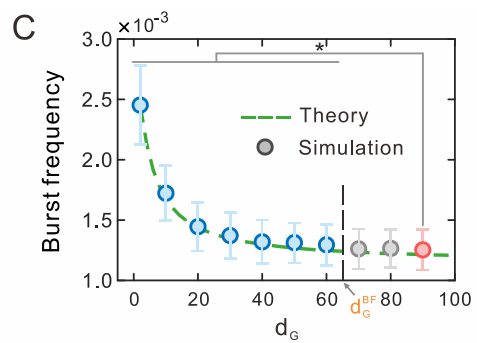

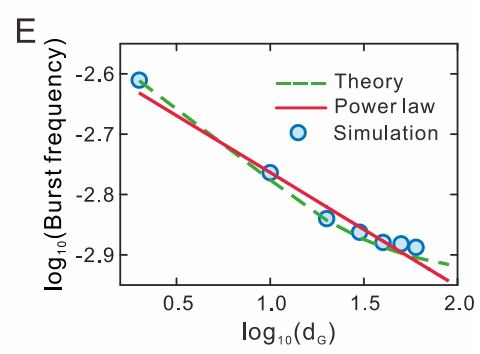

Fig. 4. Effect of E-P genomic distance on transcriptional bursting kinetics. $(A)$ A schematic diagram shows the increment of E-P genomic distance $d_{\mathrm{G}}$. (B) Influence of $d_{\mathrm{G}}$ on burst size. Dashed green line represents the theoretical result (Eq. [2]) whereas circles show numerical simulations. The blue circles indicate significant differences compared to the end data point (red) via Kruskal-Wallis nonparametric test, while the gray circles indicate no significance. $(C)$ Influence of $d_{\mathrm{G}}$ on burst frequency, where the meanings of all symbols are the same as those in $(B)$. (D) Log-log plot shows the relationship between mean burst size and $d_{\mathrm{G}}$. Blue circles are the data points with significant differences in $(B)$. The red solid line indicates the power-law approximation (Eq. [5]) with the scaling exponent $S_{\mathrm{BS}}=-0.16$. Meanings of the symbols are also similar to those in $(B)$. (E) Log-log plot shows the relationship between burst frequency and $d_{\mathrm{G}}$. Meanings of the symbols are similar to those in $(D)$ but the scaling exponent is $S_{\mathrm{BF}}=-0.19$. $(F) \log -\log$ plot of experimental data from (53).

The above biphasic behaviors have been also verified by experimental results. First, quantitative living-imaging methods showed that large E-P genomic distance between sna shadow enhancer and its target gene significantly diminishes the levels of BS and BF in Drosophila $(16,54)$, in accordance with our prediction on what $\mathrm{BS}$ and $\mathrm{BF}$ are monotonically decreasing with increasing $d_{\mathrm{G}}$. Second, the mRNA expression level, which approximately equals to the product of BS and the BF (91), is an approximately linear function of smaller E-P genomic distance $d_{\mathrm{G}}$ in logarithmic scale (53) (Fig. 4F). Third, now that a monotonically decreasing transcription level does not drop to zero, the case that the level remains at a low level for large $d_{\mathrm{G}}$ could happen (53). In a word, the E-P genomic distance impacts the mRNA expression level in a biphasic and piecewise power manner as 
described in Eq. [5] (53).

\section{Mutual information reveals the dependence of bursting kinetics on E-P communication}

In the above sections, we have used the average method, i.e., calculating the mean BS and BF (the reciprocal of mean $\mathrm{CT}$ ) that only considers the mean information of BS's and CT's distribution, to study the impact of E-P communication on bursting kinetics. Here, we investigate bursting kinetics from a perspective of information transmission. The signaling pathway of transcriptional regulation transmits upstream E-P communication signal distribution $p_{D S}\left(d_{\mathrm{S}}\right)$ to downstream transcriptional output distribution $p_{X}(x)$ (Fig. 5A, SI Appendix, Fig. S13). The noisy "promoter channel" limits the fidelity of this information transduction. To better understand the ability of E-P communication to regulate $\mathrm{BS}$ and $\mathrm{BF}$, here we use Shannon mutual information to measure how much information about the E-P communication is encoded in bursting kinetics (93, 94). Mutual information $\mathrm{MI}(X, D S)$ measured in bits is defined as

$$
\operatorname{MI}(X, D S)=\int_{0}^{+\infty} \int_{0}^{+\infty} p_{X, D S}\left(x, d_{\mathrm{S}}\right) \log _{2}\left(\frac{p_{X, D S}\left(x, d_{\mathrm{S}}\right)}{p_{X}(x) p_{D S}\left(d_{\mathrm{S}}\right)}\right) \mathrm{d} x \mathrm{~d} d_{\mathrm{S}}
$$

where $p_{X}(x)$ with $X \in\{B S, C T, O F F, O N\}$ represent BS distribution, CT distribution, OFF and ON dwell-time distributions, respectively (SI Appendix, Fig. S13). The mutual information defined in such a manner can capture the contributions of all the aspects of transcriptional output distributions, not just the mean values.

Based on the computation of $\mathrm{MI}(X, D S)$ in a wide range of $k_{\mathrm{EP}}$, we find that $\mathrm{MI}(B S, D S)$ is much smaller than $\mathrm{MI}(C T, D S)$ (Figs. 5B and 5C), implying that $\mathrm{BF}$ transmits more information than BS. Moreover, $\mathrm{MI}(O N, D S)$ is two-order smaller than $\mathrm{MI}(O F F, D S)$, indicating that $\mathrm{ON}$ dwell-time is insensitive when responding to the transduced information (Fig. 5C, red dashed line). Additionally, all the $\mathrm{MI}(X, D S)$ in Figs. 5B and 5C exhibit first an increase and then a decrease, indicating that the information transduction capacity is tunable and there exists an optimal $k_{\mathrm{EP}}$ that maximizes the $\mathrm{MI}(X, D S)$. 
A

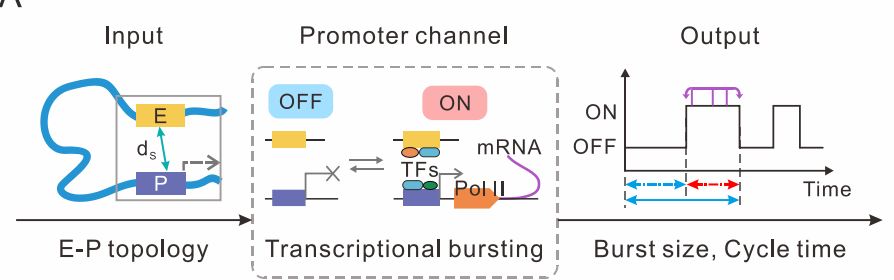

B

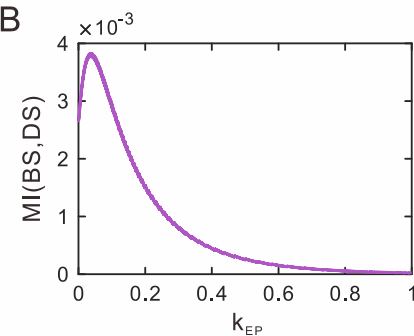

C

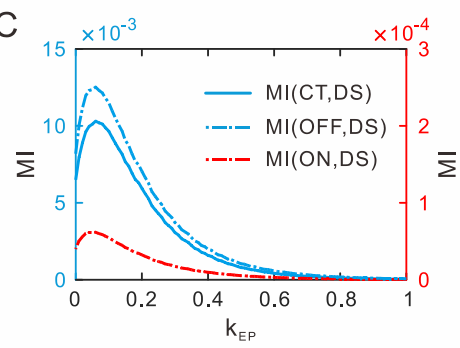

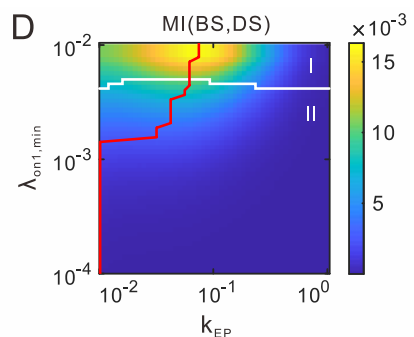

$\mathrm{E}$

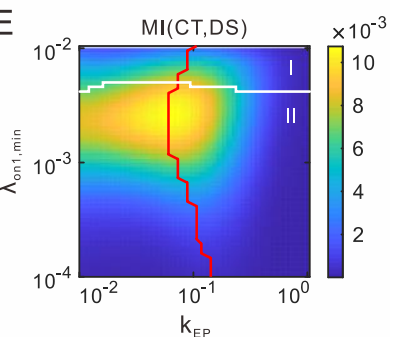

Fig. 5. Mutual information reveals the effect of E-P communication on bursting kinetics. $(A)$

An information theoretic framework used to study the effect of input E-P topology to bursting output. The promoter governing transcriptional bursting can be considered a noisy channel. (B) Mutual information between E-P spatial distance and burst size $(\mathrm{MI}(D S, B S))$ as a function of E-P communication strength $k_{\mathrm{EP}}$. (C) Mutual information between E-P spatial distance and cycle time (or OFF time, ON time) as a function of $k_{\mathrm{EP}}$. (D-E) Heatmap shows the effect of $k_{\mathrm{EP}}$ and minimum rate $\lambda_{\text {onl,min }}$ on $\operatorname{MI}(D S, B S)(D)$ and on $\operatorname{MI}(D S, C T)(E)$. The red line shows the values of $k_{\mathrm{EP}}$ obtained from the MMIs with different $\lambda_{\text {onl,min }}$. The white line is the separatrix between the values of $\operatorname{MI}(D S, B S)$ and $\operatorname{MI}(D S, C T)$. Region I is the region of $\operatorname{MI}(D S, B S)>\operatorname{MI}(D S, C T)$ and region II is the region of $\mathrm{MI}(D S, B S)<\mathrm{MI}(D S, C T)$.

Note that the maximum mutual information (MMI) measures the maximum information transmission capacity over all possible signal distributions. Obviously, the MMI exists in Figs. 5B and $5 \mathrm{C}$, where $k_{\mathrm{EP}}=0.05$ for $\mathrm{MI}(B S, D S)$ and $k_{\mathrm{EP}}=0.07$ for $\mathrm{MI}(C T, D S)$. To ascertain whether or not such an optimal $k_{\mathrm{EP}}$ always exists, we let key parameters change in wide ranges and find that there is indeed a critical $k_{\mathrm{EP}}$ maximizing the $\mathrm{MI}(X, D S)$ shown in Figs. 5D and 5E and SI Appendix, Fig. S18. In addition, the MMIs for BS and CT correspond to different $k_{\mathrm{EP}}$ values (see red lines in Figs. 5D and 5E). This asynchronous maximization indicates that the signal transduction pathway is BS- or BF-specific. In particular, the observation that $\operatorname{MI}(D S, B S)$ is smaller than $\operatorname{MI}(D S, C T)$ in region II (Fig. 5D and 5E, the region below the white line) indicates 
that BF transmits more E-P communication information than BS, and consequently, E-P communication mainly regulates BF rather than BS.

\section{Discussion}

Imaging studies, high-resolution chromatin conformation maps, and genome-wide occupancy profile of architectural proteins have revealed that genome topology encoding E-P communication information is tightly correlated with gene expression through complex regulatory layers, creating various possible transcriptional burst phenotypes. The prediction of these phenotypes requires a quantitative understanding of transcriptional bursting mechanisms underlying the influence of genomic architectures. In this paper, we have proposed a multiscale model, which integrates 3D the information on chromatin dynamics into transcription bursting, to shed light on the pivotal role of E-P spatial communication in the control of transcriptional bursting kinetics.

We have established a general modeling framework that captures salient features of intra-nuclear transcriptional bursting processes. First, the generalized Rouse model, which can well describe chromatin motion, including E-P communication, was used to model chromatin dynamics (64-67). Second, the four-state model of gene transcription adopted here captured important events occurring in transcriptional processes. Two ON states in our model can describe not only the production of nascent mRNA accompanied by the change of promoter states but also the traveling ratio property reflecting the Pol II pause release rate (see SI Appendix E3, Fig. S18), two essential characteristics distinguishing from previous models. Third, an input-output relation was proposed to link upstream chromatin configurations to downstream gene transcription where the necessity of E-P proximity (18) is well described in our model.

Our analysis has given a clear answer to the question of which of BS and BF is modulated more than the other by E-P communication. In contrast to the transcriptome-wide inference that showed the important role of E-P communication in controlling $\mathrm{BF}(92,95,96)$, we have theoretically confirmed that enhancer mainly modulates BF rather than BS (Fig. 3B), which is in agreement with observations of most experiments $(16,17,53,90,97,98)$. Moreover, we have given a theoretical estimate on the effect of the enhancer on $\mathrm{BF}$ and $\mathrm{BS}$, which is based on directly calculating the ratio of the maximum and minimum values of BS and BF experimental available. In addition, from the view of mutual information, we have shown that transcriptional bursting kinetics are regulated by 
transmitting more information to $\mathrm{BF}$, which further verifies the importance of enhancers in modulating BF.

Our results allow us to make important predictions about how upstream chromatin dynamics affect downstream transcriptional bursting kinetics. First, we have shown that E-P communication strength $k_{\mathrm{EP}}$, a key parameter in our model, modulates BS and BF in power-law manners. This power-law behavior was also verified by experimental data $(16,17,51)$. Second, we have demonstrated that E-P genomic distance $d_{\mathrm{G}}$, another vital parameter in our model, modulates BS and $\mathrm{BF}$ in biphasic fashions. This biphasic phenomenon was also verified by recent experimental observations (53). The opposite change trends for the effects of $k_{\mathrm{EP}}$ and $d_{\mathrm{G}}$ on bursting kinetics showed that the combination of $k_{\mathrm{EP}}$ and $d_{\mathrm{G}}$ would be a flexible regulation strategy that can provide insights into complex mechanisms of biological processes in organisms. We have shown that different gene state transition rates lead to different scaling exponents in power-law behaviors of BS and BF, indicating that the regulatory logics shaping differential signal transduction pathways are cell-specific and even gene-specific (99). Third, using mutual information to measure how upstream E-P communication dynamically and stochastically regulates downstream transcriptional bursting kinetics would be a more reasonable way. We have shown that the promoter information transduction capacity is tunable, and the MMI can be obtained by adjusting E-P communication strength $k_{\mathrm{EP}}$.

Our results are not limited to specific genes in Drosophila but may be applied to other organisms. The experimental data from different organisms such as a mouse model can be also well fitted (Figs. 3E-F and 4F) (17, 53), indicating the extensibility of our results. Importantly, our modeling framework can also be extended to more complex situations. For example, some experimental studies reported that transcription can affect chromatin structure $(68,69)$, and we can incorporate this feedback to our model by modifying the gene-state-dependent drift function $\boldsymbol{V}(\boldsymbol{r}, \boldsymbol{s} ; t)$ in Eq. [1]. In addition, our modeling of chromatin motion is not limited to one pair E-P communication. The potential applications may include the cases of multiple enhancers to one promoter (70), one enhancer to multiple promoters (16), or super-enhancers (100). Finally, using multi-state models of gene expression to extract insights from enormous experimental data and complex biological phenomena is impressing. Our four-state model is not the default option, and we may adjust the 
form of the downstream transcription model to include more complex biological processes such as mRNA splices (101) and cell cycle (102).

Finally, we point out that our theoretical model, which aims to develop a general modeling framework to study 4D transcriptional bursting kinetics, may provide an opportunity for a dialogue between theoretical studies and biological experiments. We envision that our modeling framework will be useful for biophysical analysis of broader in vivo cellular processes.

\section{Acknowledgments}

This work was supported by grants 12171494, 11931019, and 11775314 from Natural Science Foundation of P. R. China, and by grants 019B110233002, 202007030004 from Key-Area Research and Development Program of Guangzhou, P.R. China.

\section{Reference}

1. T. Misteli, The self-organizing genome: Principles of genome architecture and function. Cell 183, 28-45 (2020).

2. J. Rodriguez, D. R. Larson, Transcription in living cells: Molecular mechanisms of bursting. Annu. Rev. Biochem. 89, 189-212 (2020).

3. E. Tunnacliffe, J. R. Chubb, What is a transcriptional burst? Trends Genet. 36, 288-297 (2020).

4. I. Golding, J. Paulsson, S. M. Zawilski, E. C. Cox, Real-time kinetics of gene activity in individual bacteria. Cell 123, 1025-1036 (2005).

5. A. Raj, C. S. Peskin, D. Tranchina, D. Y. Vargas, S. Tyagi, Stochastic mRNA synthesis in mammalian cells. PLoS Biol. 4, e309 (2006).

6. J. R. Chubb, T. Trcek, S. M. Shenoy, Transcriptional pulsing of a developmental gene. Curr. Biol. 16, (2006).

7. J. R. Chubb, T. B. Liverpool, Bursts and pulses: Insights from single cell studies into transcriptional mechanisms. Curr. Opin. Genet. Dev. 20, 478-484 (2010).

8. D. M. Suter et al., Mammalian genes are transcribed with widely different bursting kinetics. Science 332, 472-474 (2011).

9. M. I. Robson, A. R. Ringel, S. Mundlos, Regulatory landscaping: How enhancer-promoter communication is sculpted in 3D. Mol. Cell 74, 1110-1122 (2019).

10. M. A. Zabidi, A. Stark, Regulatory enhancer-core-promoter communication via transcription factors and cofactors. Trends Genet. 32, 801-814 (2016).

11. R. Stadhouders, G. Filion, T. Graf, Transcription factors and 3D genome conformation in cell-fate decisions. Nature 569, 345-354 (2019).

12. R. Andersson, A. Sandelin, Determinants of enhancer and promoter activities of regulatory elements. Nat. Rev. Genet. 21, 71-87 (2020).

13. S. Schoenfelder, P. Fraser, Long-range enhancer-promoter contacts in gene expression control. Nat. Rev. Genet. 20, 437-455 (2019).

14. V. Haberle, A. Stark, Eukaryotic core promoters and the functional basis of transcription initiation. 
Nat. Rev. Mol. Cell Biol. 19, 621-637 (2018).

15. L. A. Lettice et al., A long-range Shh enhancer regulates expression in the developing limb and fin and is associated with preaxial polydactyly. Hum. Mol. Genet. 12, 1725-1735 (2003).

16. T. Fukaya, B. Lim, M. Levine, Enhancer control of transcriptional bursting. Cell 166, 358-368 (2016).

17. C. R. Bartman, S. C. Hsu, C. C. Hsiung, A. Raj, G. A. Blobel, Enhancer regulation of transcriptional bursting parameters revealed by forced chromatin looping. Mol. Cell 62, 237-247 (2016).

18. H. Chen et al., Dynamic interplay between enhancer-promoter topology and gene activity. Nat. Genet. 50, 1296-1303 (2018).

19. J. Rodriguez et al., Intrinsic dynamics of a human gene reveal the basis of expression heterogeneity. Cell 176, 213-226 (2019).

20. H. D. Ou et al., ChromEMT: Visualizing 3D chromatin structure and compaction in interphase and mitotic cells. Science 357, eaag0025 (2017).

21. J. Li et al., Single-gene imaging links genome topology, promoter-enhancer communication and transcription control. Nat. Struct. Mol. Biol. 27, 1032-1040 (2020).

22. J.-H. Su, P. Zheng, S. S. Kinrot, B. Bintu, X. Zhuang, Genome-scale imaging of the 3D organization and transcriptional activity of chromatin. Cell 182, 1641-1659 (2020).

23. A. M. C. Gizzi et al., Microscopy-based chromosome conformation capture enables simultaneous visualization of genome organization and transcription in intact organisms. Mol. Cell 74, 212-222 (2019).

24. E. H. Finn, T. Misteli, Molecular basis and biological function of variability in spatial genome organization. Science 365, eaaw9498 (2019).

25. Q. Szabo, F. Bantignies, G. Cavalli, Principles of genome folding into topologically associating domains. Sci. Adv. 5, eaaw1668 (2019).

26. C. H. Bohrer, D. R. Larson, The stochastic genome and its role in gene expression. Cold Spring Harb. Perspect. Biol. 13, a040386 (2021).

27. M. R. Hübner, M. A. Eckersley-Maslin, D. L. Spector, Chromatin organization and transcriptional regulation. Curr. Opin. Genet. Dev. 23, 89-95 (2013).

28. A. M. Oudelaar, D. R. Higgs, The relationship between genome structure and function. Nat. Rev. Genet. 22, 154-168 (2021).

29. B. Lim, T. Heist, M. Levine, T. Fukaya, Visualization of transvection in living Drosophila embryos. Mol. Cell 70, 287-296 (2018).

30. B. Lim, M. S. Levine, Enhancer-promoter communication: Hubs or loops? Curr. Opin. Genet. Dev. 67, 5-9 (2021).

31. E. E. M. Furlong, M. Levine, Developmental enhancers and chromosome topology. Science 361, 1341-1345 (2018).

32. V. Shahrezaei, P. S. Swain, Analytical distributions for stochastic gene expression. Proc. Natl. Acad. Sci. U.S.A. 105, (2008).

33. N. Kumar, T. Platini, R. V. Kulkarni, Exact distributions for stochastic gene expression models with bursting and feedback. Phys. Rev. Lett. 113, (2014).

34. Z. Wang, Z. Zhang, T. Zhou, Exact distributions for stochastic models of gene expression with arbitrary regulation. Sci. China Math. 63, 485-500 (2020).

35. A. M. Corrigan, E. Tunnacliffe, D. Cannon, J. R. Chubb, A continuum model of transcriptional bursting. eLife 5, e13051 (2016). 
36. J. M. Pedraza, J. Paulsson, Effects of molecular memory and bursting on fluctuations in gene expression. Science 319, 339-343 (2008).

37. J. Zhang, T. Zhou, Markovian approaches to modeling intracellular reaction processes with molecular memory. Proc. Natl. Acad. Sci. U.S.A. 116, 23542-23550 (2019).

38. J. Peccoud, B. Ycart, Markovian modeling of gene-product synthesis. Theor. Popul. Biol. 48, $222-$ 234 (1995).

39. G. Neuert et al., Systematic identification of signal-activated stochastic gene regulation. Science 339, 584-587 (2013).

40. T. Liu, J. Zhang, T. Zhou, Effect of interaction between chromatin loops on cell-to-cell variability in gene expression. PLoS Comput. Biol. 12, e1004917 (2016).

41. J. Zhang, T. Zhou, Promoter-mediated transcriptional dynamics. Biophys. J. 106, 479-488 (2014).

42. D. L. Jones, R. C. Brewster, R. Phillips, Promoter architecture dictates cell-to-cell variability in gene expression. Science 346, 1533-1536 (2014).

43. C. V. Harper et al., Dynamic analysis of stochastic transcription cycles. PLoS Biol. 9, e1000607 (2011).

44. J. P. Bothma et al., Dynamic regulation of eve stripe 2 expression reveals transcriptional bursts in living Drosophila embryos. Proc. Natl. Acad. Sci. U.S.A. 111, 10598-10603 (2014).

45. K. Tantale et al., A single-molecule view of transcription reveals convoys of RNA polymerases and multi-scale bursting. Nat. Commun. 7, 12248 (2016).

46. V. Sood, T. Misteli, The stochastic nature of genome organization and function. Curr. Opin. Genet. Dev. 72, 45-52 (2022).

47. J. Dekker, L. Mirny, The 3D genome as moderator of chromosomal communication. Cell 164, 11101121 (2016).

48. A. Elimelech, R. Y. Birnbaum, From 3D organization of the genome to gene expression. Curr. Opin. Syst. Biol. 22, 22-31 (2020).

49. I. Brouwer, T. L. Lenstra, Visualizing transcription: Key to understanding gene expression dynamics. Curr. Opin. Chem. Biol. 51, 122-129 (2019).

50. J. Y. Xiao, A. Hafner, A. N. Boettiger, How subtle changes in 3D structure can create large changes in transcription. eLife 10, e64320 (2021).

51. A. Senecal et al., Transcription factors modulate c-Fos transcriptional bursts. Cell Rep. 8, 75-83 (2014).

52. C. R. Bartman et al., Transcriptional burst initiation and polymerase pause release are key control points of transcriptional regulation. Mol. Cell 73, 519-532 (2019).

53. J. Zuin et al., Nonlinear control of transcription through enhancer-promoter interactions. bioRxiv [Preprint] (2021). https://doi.org/10.1101/2021.04.22.440891

54. M. Yokoshi, K. Segawa, T. Fukaya, Visualizing the role of boundary elements in enhancer-promoter communication. Mol. Cell 78, 224-235 (2020).

55. N. C. Lammers, Y. J. Kim, J. Zhao, H. G. Garcia, A matter of time: Using dynamics and theory to uncover mechanisms of transcriptional bursting. Curr. Opin. Cell Biol. 67, 147-157 (2020).

56. C. P. Johnstone, N. B. Wang, S. A. Sevier, K. E. Galloway, Understanding and engineering chromatin as a dynamical system across length and timescales. Cell Syst. 11, 424-448 (2020).

57. T. Heist, T. Fukaya, M. Levine, Large distances separate coregulated genes in living Drosophila embryos. Proc. Natl. Acad. Sci. U.S.A. 116, 15062-15067 (2019).

58. W. Shao, J. Zeitlinger, Paused RNA polymerase II inhibits new transcriptional initiation. Nat. Genet. 
49, 1045-1051 (2017).

59. A. R. Krebs et al., Genome-wide single-molecule footprinting reveals high RNA polymerase II turnover at paused promoters. Mol. Cell 67, 411-422 (2017).

60. T. Henriques et al., Widespread transcriptional pausing and elongation control at enhancers. Genes Dev. 32, 26-41 (2018).

61. C. W. Gardiner, Handbook of stochastic methods for physics, chemistry and the natural sciences. (Springer, Berlin, 1983).

62. J. Dekker et al., The 4D nucleome project. Nature 549, 219-226 (2017).

63. M. A. Marti-Renom et al., Challenges and guidelines toward 4D nucleome data and model standards. Nat. Genet. 50, 1352-1358 (2018).

64. M. Doi, S. Edwards, The theory of polymer dynamics. (Oxford University Press, New York 1988).

65. M. Barbieri et al., Complexity of chromatin folding is captured by the strings and binders switch model. Proc. Natl. Acad. Sci. U.S.A. 109, 16173-16178 (2012).

66. O. Shukron, D. Holcman, Transient chromatin properties revealed by polymer models and stochastic simulations constructed from Chromosomal Capture data. PLoS Comput. Biol. 13, e1005469 (2017).

67. L. Giorgetti et al., Predictive polymer modeling reveals coupled fluctuations in chromosome conformation and transcription. Cell 157, 950-963 (2014).

68. S. Heinz et al., Transcription elongation can affect genome 3D structure. Cell 174, 1522-1536 (2018).

69. G. A. Busslinger et al., Cohesin is positioned in mammalian genomes by transcription, CTCF and Wapl. Nature 544, 503-507 (2017).

70. B. He, C. Chen, L. Teng, K. Tan, Global view of enhancer-promoter interactome in human cells. Proc. Natl. Acad. Sci. U.S.A. 111, E2191-E2199 (2014).

71. A. Bancaud et al., Molecular crowding affects diffusion and binding of nuclear proteins in heterochromatin and reveals the fractal organization of chromatin. EMBO J. 28, 3785-3798 (2009).

72. Y. Zhang, O. K. Dudko, First-Passage Processes in the Genome. Annual review of biophysics $\mathbf{4 5}$, 117-134 (2016).

73. C. Engl, G. Jovanovic, R. D. Brackston, I. Kotta-Loizou, M. Buck, The route to transcription initiation determines the mode of transcriptional bursting in E. coli. Nat. Commun. 11, 2422 (2020).

74. N. J. Fuda, M. B. Ardehali, J. T. Lis, Defining mechanisms that regulate RNA polymerase II transcription in vivo. Nature 461, 186-192 (2009).

75. F. X. Chen et al., PAF1 regulation of promoter-proximal pause release via enhancer activation. Science 357, 1294-1298 (2017).

76. P. Thomas, N. Popović, R. Grima, Phenotypic switching in gene regulatory networks. Proc. Natl. Acad. Sci. U.S.A. 111, 6994-6999 (2014).

77. N. Friedman, L. Cai, X. S. Xie, Linking stochastic dynamics to population distribution: An analytical framework of gene expression. Phys. Rev. Lett. 97, 168302 (2006).

78. G. Nicoletti, D. M. Busiello, Mutual information disentangles interactions from changing environments. Phys. Rev. Lett. 127, 228301 (2021).

79. J. M. Alexander et al., Live-cell imaging reveals enhancer-dependent Sox 2 transcription in the absence of enhancer proximity. eLife 8, e41769 (2019).

80. D. I. Cattoni et al., Single-cell absolute contact probability detection reveals chromosomes are organized by multiple low-frequency yet specific interactions. Nat. Commun. 8, 1753 (2017).

81. K. N. Rybakova et al., Multiplex eukaryotic transcription (in) activation: timing, bursting and cycling of a ratchet clock mechanism. PLoS Comput. Biol. 11, e1004236 (2015). 
82. M. Kandhavelu, A. Häkkinen, O. Yli-Harja, A. S. Ribeiro, Single-molecule dynamics of transcription of the lar promoter. Phys. Biol. 9, 026004 (2012).

83. A. E. Douglas, The Drosophila model for microbiome research. Lab animal 47, 157-164 (2018).

84. B. H. Jennings, Drosophila-a versatile model in biology \& medicine. Mater. Today 14, 190-195 (2011).

85. J. P. Bothma et al., Enhancer additivity and non-additivity are determined by enhancer strength in the Drosophila embryo. eLife 4, e07956 (2015).

86. M. W. Perry, A. N. Boettiger, J. P. Bothma, M. Levine, Shadow enhancers foster robustness of Drosophila gastrulation. Curr. Biol. 20, 1562-1567 (2010).

87. D. A. Stavreva et al., Dynamics of chromatin accessibility and long-range interactions in response to glucocorticoid pulsing. Genome Res. 25, 845-857 (2015).

88. M. Murata, P. Gong, K. Suzuki, S. Koizumi, Differential metal response and regulation of human heavy metal - inducible genes. J. Cell. Physiol. 180, 105-113 (1999).

89. C. Fritzsch et al., Estrogen-dependent control and cell-to-cell variability of transcriptional bursting. Mol. Syst. Biol. 14, e7678 (2018).

90. D. R. Larson et al., Direct observation of frequency modulated transcription in single cells using light activation. eLife 2, e00750 (2013).

91. L. Li, R. Waymack, M. Gad, Z. Wunderlich, Two promoters integrate multiple enhancer inputs to drive wild-type knirps expression in the Drosophila melanogaster embryo. Genetics 219, iyab154 (2021).

92. A. J. M. Larsson et al., Genomic encoding of transcriptional burst kinetics. Nature 565, 251-254 (2019).

93. C. E. Shannon, A mathematical theory of communication. Bell Syst. Tech. J. 27, 379-423 (1948).

94. T. M. Cover, Elements of information theory (John Wiley \& Sons, 1999).

95. A. J. Larsson, C. Coucoravas, R. Sandberg, B. Reinius, X-chromosome upregulation is driven by increased burst frequency. Nat. Struct. Mol. Biol. 26, 963-969 (2019).

96. H. Ochiai et al., Genome-wide kinetic properties of transcriptional bursting in mouse embryonic stem cells. Sci. Adv. 6, eaaz6699 (2020).

97. M. C. Walters et al., Enhancers increase the probability but not the level of gene expression. Proc. Natl. Acad. Sci. U.S.A. 92, 7125-7129 (1995).

98. H. G. Sutherland, D. I. Martin, E. Whitelaw, A globin enhancer acts by increasing the proportion of erythrocytes expressing a linked transgene. Mol. Cell. Biol. 17, 1607-1614 (1997).

99. A. H. Brivanlou, J. E. Darnell, Signal transduction and the control of gene expression. Science 295, 813-818 (2002).

100. S. Pott, J. D. Lieb, What are super-enhancers? Nat. Genet. 47, 8-12 (2015).

101. Z. Kan, D. States, W. Gish, Selecting for functional alternative splices in ESTs. Genome Res. 12, 1837-1845 (2002).

102. D. G. Johnson, C. L. Walker, Cyclins and cell cycle checkpoints. Annu. Rev. Pharmacol. Toxicol. 39, 295-312 (1999). 


\section{Supplementary Information}

\section{Theoretical Principles of Enhancer-Promoter Communication in Transcriptional Bursting}

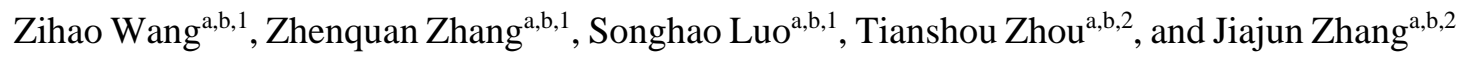

${ }^{a}$ Guangdong Province Key Laboratory of Computational Science School of Mathematics, Sun Yat-sen University, Guangzhou 510275, P. R. China

${ }^{\mathrm{b}}$ School of Mathematics, Sun Yat-Sen University, Guangzhou 510275, P. R. China

${ }^{1}$ These authors contributed equally to this work.

${ }^{2}$ To whom correspondence may be addressed.

Email: zhjiajun@mail.sysu.edu.cn or mcszhtsh@mail.sysu.edu.cn

\section{This PDF file includes:}

Supplementary Text.

Supplementary Figures.

Supplementary References. 


\section{Content}

A Introduction to background knowledge .......................................................................... 3

A1 Three-dimensional genome organization and dynamics ...........................................................

A2 Enhancer-Promoter communication ..........................................................................................

A3 Multistep transcription process .......................................................................................

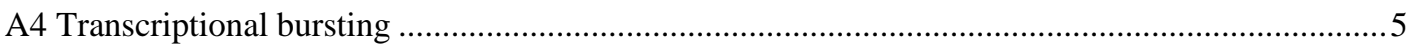

A5 Different timescales between chromatin motion and transcriptional bursting ...............................5

A6 Question: how E-P communication regulates transcriptional bursting ............................................ 6

B Differential Chapman-Kolmogorov Equation (dCKE) ................................................... 7

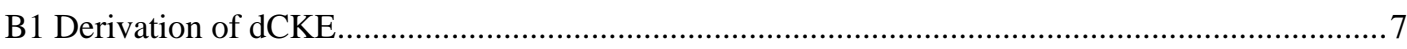

B2 Simulation algorithm for dCKE .....................................................................................

C Modelling transcriptional bursting by dCKE .......................................................... 11

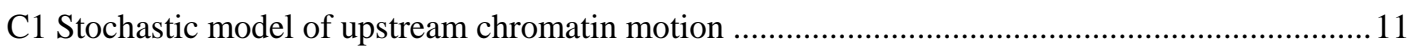

C2 Multi-state model of downstream transcriptional bursting .........................................................13

C3 Information flow from chromatin conformation to transcriptional bursting .................................17

D Simulation and statistical analysis of the whole model ....................................................... 21

E Theoretical analysis of transcriptional bursting ....................................................................... 23

E1 Timescale separation method decoupling upstream and downstream kinetics ............................23

E2 Analytical results for upstream chromatin dynamics..............................................................25

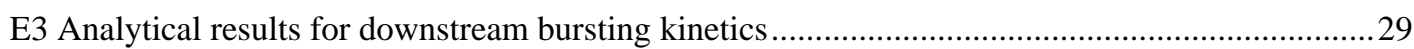

F Power laws for transcriptional bursting kinetics .................................................................... 39

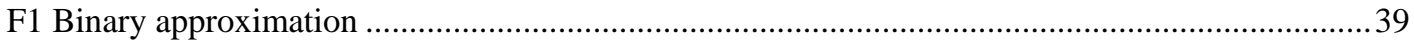

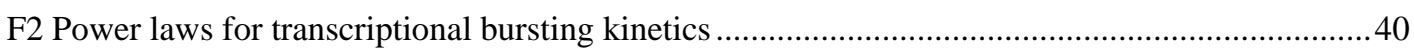

G E-P communication mainly modulates burst frequency ............................................... 45

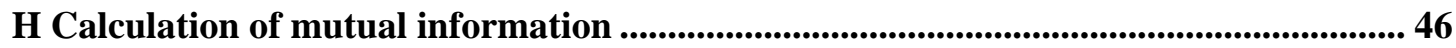

I Biologically reasonable setting of model parameter values................................................... 48

J Experimental confirmations and model predictions ...................................................53

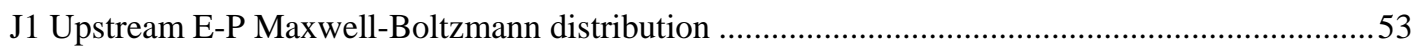

J2 Power-laws for transcriptional bursting kinetics .......................................................................5

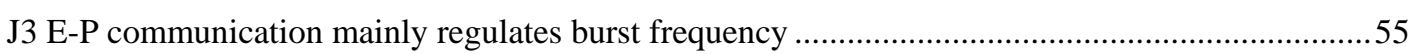

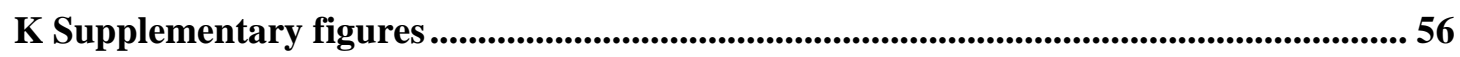

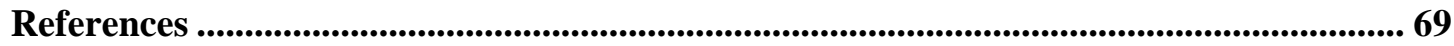




\section{A Introduction to background knowledge}

\section{A1 Three-dimensional genome organization and dynamics}

\section{Multiscale spatial organization of genome}

Genomes have complex three-dimensional (3D) architectures employed to store complete genetic information in an organism. To accommodate the genome within a confined space of cell nucleus, the genomes must be organized and compacted, with the assurance that genomic function is accurately achieved at any location and time. This multi-level 3D genomic organization is highly ordered but spatially multiscale $(1,2)$.

In spite of their architectures depending on organisms and with differently characteristic scales, genomes contain DNA, nucleosomes, fibers, loops, domains, and compartments as well as chromosomes. DNA is composed of 2 polynucleotide chains wrapped around each other to form a double helix structure. Nucleosomes made up of the octamers that are composed of corehistones are wrapped by DNA and further helices to form chromatin fibers. Chromatin fibers can be folded to form loops that typically link upstream regulatory elements such as enhancers close to the promoters to control downstream gene transcription. Also, chromatin fibers themselves can form topologically associating domains (TADs), which are genomic regions that preferentially interplay with each other. The TADs are assembled to form higher-order chromatin compartments. Chromosomes are the primary elements of genomic organization, and distinct chromosomes exist in the nucleus in the form of chromosomal territories.

\section{Stochasticity of genome organization}

Genomic organization is highly stochastic within each single cell and this stochasticity is similar to cell-to-cell variability in transcriptional outcomes (3). It has been experimentally observed that chromosomes are highly variable in structure, position, and level of crosschromosomal contacts between two homologs and even between individual cells (4). Also, there is heterogeneity in the location of TAD boundaries and the formation of chromatin loops (5-7). In particular, the stochasticity of chromatin loops propagates directly to enhancerpromoter (E-P) communication, implying that distal regulation is a source of transcriptional noise. A major challenge is to identify how an enhancer distally regulates transcription via a precise regulation mechanism under this highly stochastic genome organization. It is noted that 
3D genomic organization simultaneously possesses high order and high variability properties that are in conflict with each other. This double property is a sign that genomic organization is a self-organization, a mechanism that is universal in nature (1).

\section{A2 Enhancer-Promoter communication}

The chromatin loop structure of 3D genomes, which serves as an intermediate regulation between regulatory elements, often contributes to enhancer-promoter (E-P) communication and further gene expression (8). This E-P communication is a long-range interaction that makes it possible to spatially contact the promoter of the target gene by the distal regulatory element-enhancer, rather than to be just influenced by the proximal regulatory element. These observations raise an open question: how enhancers and promoters construct regulatory landscapes and how E-P communication is sculpted in 3D chromatin structure. See Section C3 for explanations.

\section{A3 Multistep transcription process}

Transcription is a complex multistep process, involving chromatin remodeling, recruitment of transcription factors (TFs) and RNA polymerase II (Pol II), transcription initialization, transitions between transcriptionally active and inactive states, etc. Specifically, a transcription period roughly involves the following eight steps: the first step is chromatin opening, the second step is the formation of pre-initiation complex (i.e., PIC formation), the third step is transcription initialization, the fourth step is promoter escape/clearance, the fifth step is the escape from pausing, the sixth step is productive elongation, the seventh step is another cycle of transcription, and the eighth step is transcription termination (9). In Section A5 and C2, we have also given some detailed explanations.

Concretely, E-P communication can regulate almost every step of the expression process involved in transcription initiation, including PIC formation, Pol II activation transcription and Pol II pause release. Therefore, it is essential to consider the multi-step process of transcription in investigating the impact of E-P communication on transcriptional expression. In Section C3, we have given some detailed explanations about how E-P communication regulates transcriptional bursting. 


\section{A4 Transcriptional bursting}

Bursty expression patterns, as the outputs of multi-step transcriptional processes, have been observed in multiple experiments in various systems and cell types (10-14). Many mathematical models used to describe burst phenotypes have been proposed, such as the random telegraph (ON-OFF) model (15) and three-state model (16) as well as the multi-state model (17). In fact, the random telegraph model has been widely adopted to both explain transcriptional dynamics and understand the mechanisms of whole genome transcriptional regulation (18). In addition, three-state or multi-state model have been theoretically used to explain experimental phenomena $(19,20)$.

Transcriptional bursting kinetics can be characterized by two main quantities: burst size and burst frequency. First, once the burst is initiated, the Pol II is able to load on the promoter that DNA is transcribed into mRNA. When the promoter changes from the active to the inactive states, the number of mRNAs produced in this burst can be calculated, i.e., burst size. Another quantity that determines how frequently a burst can be observed, is burst frequency. It depends on the process that precedes the initial transcription, such as E-P communication, recruitment of TFs. Understanding how genes encode molecular regulatory mechanisms of bursting (burst frequency and size) is a highly interesting matter.

\section{A5 Different timescales between chromatin motion and transcriptional bursting}

First, chromatin spatial folding and chromatin accessibility are essential for the contact of distal enhancers with their target promoters (21). The basic unit of chromatin is nucleosome and nucleosome rewrapping is the obstacle hindering the access of Pol II (22). The assembly or unwrapping time of nucleosome is about 1 second $(1 \mathrm{~s})(23,24)$. Meanwhile, the communication between enhancer and distal promoter can form on a timescale of seconds to minutes (25-27). Therefore, the timescale of chromatin motion is less than seconds. Some studies have used 1e-4 to 1e-2s (28-30) to represent the timescale of chromatin dynamic motion.

Second, transcription will be possible once the chromatin is open, and a cascade of biochemical processes will be triggered, such as TFs binding, Pol II recruitment, transcriptional elongation, etc. The transcription cycle might be more complex than the mentioned-above simple eight steps, and many questions about transcriptional bursting remain elusive. Imaging 
studies have shown that the TFs binding to DNA is a highly transient and dynamic process, occurring on the order of hundreds of milliseconds to seconds (31-34). TFs binding, especially TFIID, can distort the TATA box (35). This distortion is considered to be a marker of locating the promoter, allowing subsequent PIC assembly steps. Besides, Pol II can form clusters in vivo and its dynamics takes place in a few seconds (36). TFs recruitment and Pol II recruitment are related to E-P communication. Although TFs and Pol II can bind to enhancer and promoter and maintain the E-P communication, the E-P spatial distance is highly dynamic (37) and stochastically fluctuating on a timescale of seconds to minutes (25-27). In addition, Pol II pause is a significant event in bursting processes. Pol II pause release needs positive transcription elongation factor $b(\mathrm{P}-\mathrm{TEFb})$ to phosphorylate Ser2 and negative elongation factor (NELF) (9). Recent genome-wide studies have shown that the half-lives of Pol II pause is on a minute timescale (38). Of course, a transcriptional burst may include multiple transcription cycles (i.e., another round of transcription after Pol II pause release). Thus, transcription processes occur in discontinuous episodic bursts and can last minutes to hours (39-41). Meanwhile, multiple cycles and burst duration indicate that transcriptional bursting itself is a multiscale process (16).

\section{A6 Question: how E-P communication regulates transcriptional bursting}

Imaging studies of chromatin conformations and genome-wide occupancy data of architectural proteins have revealed that genome topology, especially E-P communication, is tightly intertwined with gene expression $(42,43)$. Here, a challenging question arises: how E$\mathrm{P}$ communication regulates transcriptional bursting. The fact that the genomic structures are stochastic (3) and that transcription process and chromatin movement are on different timescales (44) brings difficulties to address this question. In this paper, we establish an analysis framework to reveal the mechanism of transcriptional bursting and predict possible dynamic behaviors compatible with experimental observations. 


\section{B Differential Chapman-Kolmogorov Equation (dCKE)}

\section{B1 Derivation of $\mathrm{dCKE}$}

As mentioned in Section A, gene transcription is a multistep and multiscale process, involving genomic organization (including chromatin folding and E-P communication) and upstream-to-downstream regulation. To establish a general model of transcription, we assume that there are in total $N$ nucleosomes on chromatin, and denote by $r=\left(r_{i j}\right)_{N \times 3}$ the spatial positions of these nucleosomes in 3D space. In addition, we assume that the gene has in total $K$ transcriptionally active and inactive states, among which transitions occur on a slow timescale compared with chromatin motion occurring on a fast timescale. Denote by $s$ the vector of all the gene states.

Let $\boldsymbol{p}(\boldsymbol{r}, \boldsymbol{s} ; t)$ be a vector of the joint probability density functions (PDFs) that nucleosomes are in position $\boldsymbol{r}=\left[\boldsymbol{r}_{1}, \cdots, \boldsymbol{r}_{N}\right]^{\mathrm{T}}$ and the gene is in state $\boldsymbol{s}=\left[s_{1}, \cdots, s_{K}\right]^{\mathrm{T}}$ at time $t$, where $\mathrm{T}$ is transpose. Note that $\boldsymbol{p}(\boldsymbol{r}, \boldsymbol{s} ; t)=\left[\boldsymbol{p}\left(\boldsymbol{r}, s_{1} ; t\right), \cdots, \boldsymbol{p}\left(\boldsymbol{r}, s_{K} ; t\right)\right]^{\mathrm{T}}$ can be regarded as a mapping: $\mathbb{R}^{3 N} \times S \times \mathbb{R} \mapsto \mathbb{R}^{K}$ where $S=\left\{s_{1}, \cdots, s_{K}\right\}$. Based on the principle of total probability, $\boldsymbol{p}(\boldsymbol{r}, \boldsymbol{s} ; \boldsymbol{t})$ can be written as the product of the PDF $p(\boldsymbol{r} ; t): \mathbb{R}^{3 N} \times \mathbb{R} \mapsto \mathbb{R}$ and the conditional PDF (cPDF)

$$
\begin{aligned}
& \boldsymbol{p}(\boldsymbol{s} \mid \boldsymbol{r} ; t)=\left[\boldsymbol{p}\left(s_{1} \mid \boldsymbol{r} ; t\right), \cdots, \boldsymbol{p}\left(s_{K} \mid \boldsymbol{r} ; t\right)\right]^{\mathrm{T}}: S \times \mathbb{R} \mapsto[0,1]^{K} \text {, that is, } \\
& \boldsymbol{p}(\boldsymbol{r}, \boldsymbol{s} ; t)=p(\boldsymbol{r} ; t) \boldsymbol{p}(\boldsymbol{s} \mid \boldsymbol{r} ; t) .
\end{aligned}
$$

Differentiating Eq. (B1) with respect to time yields

$$
\frac{\partial \boldsymbol{p}(\boldsymbol{r}, \boldsymbol{s} ; t)}{\partial t}=\frac{\partial p(\boldsymbol{r} ; t)}{\partial t} \boldsymbol{p}(\boldsymbol{s} \mid \boldsymbol{r} ; t)+\frac{\partial \boldsymbol{p}(\boldsymbol{s} \mid \boldsymbol{r} ; t)}{\partial t} p(\boldsymbol{r} ; t) .
$$

Considering that $r \in \Omega \subset \mathbb{R}^{3 N}$ is a "fast" variable for a continuous trajectory, where $\Omega$ is a connected and bounded domain. Then, derivative $\frac{\partial p(\boldsymbol{r} ; t)}{\partial t}$ in Eq. (B2) can be formally written as

$$
\frac{\partial p(\boldsymbol{r} ; t)}{\partial t}=-\nabla_{r} \cdot \boldsymbol{J}(\boldsymbol{r}, \boldsymbol{s} ; t)
$$

where $\boldsymbol{J}(\boldsymbol{r}, \boldsymbol{s} ; t)=\boldsymbol{F}(\boldsymbol{r}, \boldsymbol{s} ; t) p(\boldsymbol{r} ; t)$ is the probability flux and $\boldsymbol{F}(\boldsymbol{r}, \boldsymbol{s} ; t)$ is a velocity field. If we only consider isotropic diffusion and friction across the region of nucleosome positions, then 
$\boldsymbol{F}(\boldsymbol{r}, \boldsymbol{s} ; t)$ can take the following generalized Fokker-Planck approximation:

$$
\boldsymbol{F}(\boldsymbol{r}, \boldsymbol{s} ; t)=\boldsymbol{V}(\boldsymbol{r}, \boldsymbol{s} ; t)-\nabla_{\boldsymbol{r}} \cdot(D \log p(\boldsymbol{r} ; t)) .
$$

The first term on the right hand side of Eq. (B4) represents the deterministic part of the velocity field. The second term is a stochastic ingredient of the velocity following Fickian diffusion with a diffusion matrix that is assumed as the form of $\boldsymbol{D}=D \boldsymbol{I}$, where $D$ is a diffusion coefficient and $\boldsymbol{I}$ is the identity matrix. If we assume that changes in gene state do not contribute to chromatin motion, the velocity field $\boldsymbol{V}(\boldsymbol{r}, \boldsymbol{s} ; t)$ can be approximated by $\boldsymbol{V}(\boldsymbol{r} ; t)$ and $\boldsymbol{V}(\boldsymbol{r} ; t)=-\frac{1}{\gamma} \nabla_{\boldsymbol{r}} U(\boldsymbol{r} ; t)$, where $U(\boldsymbol{r} ; t)$ is the energy field and $\gamma$ is a drag coefficient.

Substituting Eq. (B4) into Eq. (B3) yields

$$
\frac{\partial p(\boldsymbol{r} ; t)}{\partial t}=-\nabla_{\boldsymbol{r}} \cdot(\boldsymbol{V}(\boldsymbol{r} ; t) p(\boldsymbol{r} ; t))+\nabla_{r}^{2}(D p(\boldsymbol{r} ; t)) .
$$

Since the diffusion of a chromatin chain within the cell nucleus is bounded and is often restricted to a subcellular compartment $(\Omega)$ that would have complex geometry, it is necessary to impose constraints of probability flux $\boldsymbol{J}(\boldsymbol{r} ; t)$ on the boundary $\partial \Omega$ of $\Omega$. Here we choose a homogeneous Neumann boundary condition (no-flux), i.e., $\boldsymbol{J}(\boldsymbol{r} ; t) \cdot \boldsymbol{n}(\boldsymbol{r})=\mathbf{0}$ for all $\boldsymbol{r} \in \partial \Omega$, where $\boldsymbol{n}(\boldsymbol{r})$ is the unit outward normal to the boundary $\partial \Omega$. The Langevin equation or stochastic differential equation corresponding to Eq. (B5) is

$$
\mathrm{d} \boldsymbol{r}=\boldsymbol{V}(\boldsymbol{r} ; t) \mathrm{d} t+\sqrt{2 D} \mathrm{~d} \boldsymbol{B}(t),
$$

where $\boldsymbol{B}(t)$ are independent Brownian motions or Wiener processes (representing Gaussian white noise).

On the other hand, consider that gene state $s$ is a "slow" variable in a discrete pathway. Then, $\frac{\partial \boldsymbol{p}(\boldsymbol{s} \mid \boldsymbol{r} ; t)}{\partial t}$ features the process of gene-state switching, and can be written by a master equation:

$$
\frac{\partial p(s \mid \boldsymbol{r} ; t)}{\partial t}=\boldsymbol{W}(\boldsymbol{r} ; t) \boldsymbol{p}(\boldsymbol{s} \mid \boldsymbol{r} ; t),
$$

where $\boldsymbol{W}(\boldsymbol{r} ; t)=\left(\lambda_{i j}(\boldsymbol{r} ; t)\right)_{K \times K}$ is a nucleosome position-dependent state transition matrix, 
satisfying the conservative condition: $\sum_{i=1}^{K} \lambda_{i j}(r ; t)=0$.

Substituting Eq. (B5) and Eq. (B7) into Eq. (B2), Eq. (B1) yields the following equation

$$
\frac{\partial \boldsymbol{p}(\boldsymbol{r}, \boldsymbol{s} ; t)}{\partial t}=\left[-\nabla_{\boldsymbol{r}} \cdot(\boldsymbol{V}(\boldsymbol{r} ; t) p(\boldsymbol{r} ; t))+\nabla_{\boldsymbol{r}}^{2}(D p(\boldsymbol{r} ; t))\right] \boldsymbol{p}(\boldsymbol{s} \mid \boldsymbol{r} ; t)+\boldsymbol{W}(\boldsymbol{r} ; t) \boldsymbol{p}(\boldsymbol{s} \mid \boldsymbol{r} ; t) p(\boldsymbol{r} ; t) .
$$

It is reasonable to assume that tiny changes in nucleosome coordinates do not alter gene state, implying that the derivative of conditional probability, $\nabla_{r} \boldsymbol{p}(s \mid \boldsymbol{r} ; t)$, approximately equals zero partly because of the time interval of downstream gene state transition is generally longer than that of upstream chromatin motion (44). Thus, according to Eq. (B1), we can further obtain the following equation

$$
\frac{\partial \boldsymbol{p}(\boldsymbol{r}, \boldsymbol{s} ; t)}{\partial t}=-\nabla_{\boldsymbol{r}} \cdot\left(\boldsymbol{p}(\boldsymbol{r}, \boldsymbol{s} ; t) \boldsymbol{V}(\boldsymbol{r} ; t)^{\mathrm{T}}\right)+\nabla_{r}^{2}(\boldsymbol{D} \boldsymbol{p}(\boldsymbol{r}, \boldsymbol{s} ; t))+\boldsymbol{W}(\boldsymbol{r} ; t) \boldsymbol{p}(\boldsymbol{r}, \boldsymbol{s} ; t) .
$$

Summing all states $s$ on both sides of Eq. (B9), we can in turn derive Eq. (B5). Also, integrating all positions $r$ over $\Omega$ on both sides of Eq. (B9) yields the following integral equation for $\boldsymbol{p}(\boldsymbol{r}, \boldsymbol{s} ; t)$

$$
\frac{\partial}{\partial t} \int_{\Omega} p(\boldsymbol{r}, \boldsymbol{s} ; t) d \boldsymbol{r}=\int_{\Omega} \mathbf{W}(\boldsymbol{r} ; t) \boldsymbol{p}(\boldsymbol{r}, \boldsymbol{s} ; t) d \boldsymbol{r} .
$$

\section{B2 Simulation algorithm for dCKE}

Since Eq. (B9) involves the spatial derivative $\nabla_{r}$, the traditional Gillespie stochastic simulation algorithm (45) cannot be directly applied. Here we propose an improved version of this algorithm to solve Eq. (B9).

Assume that there are $L$ reactions involved in the gene-state switching process. The $l$ th reaction propensity is denoted by $a_{l}(\boldsymbol{r}, \boldsymbol{s} ; t), \quad l=1,2, \cdots, L$, and the total reaction propensity is calculated according to $a_{\mathrm{tot}}(\boldsymbol{r}, \boldsymbol{s} ; t)=\sum_{l=1}^{L} a_{l}(\boldsymbol{r}, \boldsymbol{s} ; t)$. Note that $s$ is the vector of all gene states, but in the following algorithm, we set $K$-dimensional state vector $s=[0, \cdots, 0,1,0, \cdots, 0]^{\mathrm{T}}$ when the current state is $j$ in numerical simulation. Element $v_{i j}$ of stoichiometric matrix $\left(v_{i j}\right)_{K \times L}$ denotes the net change in gene state $j$ due to each reaction $i$, and $v_{\mu}$ is the $\mu$ th column of $\left(v_{i j}\right)$. Let $H(\boldsymbol{r}, \boldsymbol{s} ; t)$ is the survival probability that chromatin position is $\boldsymbol{r}$ and 
gene state is $s$ state at time $t$. The main steps for solving Eq. (B9) are listed below:

(1) Set the initial state as $\boldsymbol{r}_{0}=\boldsymbol{r}\left(t_{0}\right), \boldsymbol{s}_{0}=\boldsymbol{s}\left(t_{0}\right)$.

(2) Generate two random variables $u_{1}$ and $u_{2}$ distributed uniformly in interval $(0,1)$.

(3) Integrate the system of stochastic differential equations

$$
\left\{\begin{array}{l}
\mathrm{d} \boldsymbol{r}=\boldsymbol{V}(\boldsymbol{r} ; t) \mathrm{d} t+\sqrt{2 \boldsymbol{D}} \mathrm{d} \boldsymbol{B}(t), \\
\mathrm{d} H(\boldsymbol{r}, \boldsymbol{s} ; t)=-a_{\mathrm{tot}}(\boldsymbol{r}, \boldsymbol{s} ; t) H(\boldsymbol{r}, \boldsymbol{s} ; t) \mathrm{d} t, \\
\boldsymbol{r}\left(t_{i}\right)=\boldsymbol{r}_{i}, H\left(t_{i}\right)=1,
\end{array}\right.
$$

from time points $t_{i}$ to $t_{i}+\tau_{i}$, and with the stopping condition $H\left(\boldsymbol{r}_{i}, \boldsymbol{s}_{i} ; t_{i}+\tau_{i}\right)=u_{1}$.

(4) Update time and position: $t_{i+1}=t_{i}+\tau_{i}, \boldsymbol{r}_{i+1}=\boldsymbol{r}\left(t_{i+1}\right)$.

(5) Choose $\mu$ such that

$$
\sum_{l=1}^{\mu-1} a_{l}\left(\boldsymbol{r}_{i+1}, \boldsymbol{s}_{i} ; t_{i+1}\right)<u_{2} a_{\mathrm{tot}}\left(\boldsymbol{r}_{i+1}, \boldsymbol{s}_{i} ; t_{i+1}\right) \leq \sum_{l=1}^{\mu} a_{l}\left(\boldsymbol{r}_{i+1}, \boldsymbol{s}_{i} ; t_{i+1}\right) .
$$

(6) Update promoter state: $\boldsymbol{s}_{i+1}=\boldsymbol{s}_{i}+\boldsymbol{v}_{\mu}$.

(7) Reiterate the system from step (2) with a new state until a given largest time $t_{\max }$ is reached.

By the above algorithm steps, we can generate sample trajectories of the system described by Eq. (B9). 


\section{Modelling transcriptional bursting by dCKE}

\section{C1 Stochastic model of upstream chromatin motion}

We model chromatin as a folded polymer chain, a collection of monomers connected by springs. We assume that a monomer represents a nucleosome, and a nucleosome represents an enhancer whereas another nucleosome represents a promoter. Also, we posit that there are only one enhancer and one promoter on the chromatin (i.e., polymer) although multiple enhancers may exist, and neglect attraction or repulsion between other monomers in the chain except for the communication between the enhancer and the promoter. Then, the chromatin motion can be described by the Langevin equation (i.e., (B6))

$$
\mathrm{d} \boldsymbol{r}=-\frac{1}{\gamma} \nabla_{\boldsymbol{r}} U(\boldsymbol{r} ; t) \mathrm{d} t+\sqrt{2 D} \mathrm{~d} \boldsymbol{B}(t)
$$

where $U(\boldsymbol{r} ; t)$ is the total potential of the polymer chain at time $t$. We make decomposition $U(\boldsymbol{r} ; t)=U_{\mathrm{NN}}(\boldsymbol{r} ; t)+U_{\mathrm{EP}}(\boldsymbol{r} ; t)$, where $U_{\mathrm{NN}}(\boldsymbol{r} ; t)$ is the potential of the successive monomers and $U_{\mathrm{EP}}(\boldsymbol{r} ; t)$ is that of the E-P communication.

To simplify but without loss of generality, harmonic springs with stiffness $k_{\mathrm{NN}}$ are used between the monomers of nearest-neighbors along the chain. Thus, the potential of the successive monomers is

$$
U_{\mathrm{NN}}\left(\boldsymbol{r}_{1}, \ldots, \boldsymbol{r}_{N}\right)=\frac{1}{2} \sum_{j=1}^{N-1} k_{\mathrm{NN}}\left(\left|\boldsymbol{r}_{j}-\boldsymbol{r}_{j+1}\right|-l_{0}\right)^{2}
$$

where $\boldsymbol{r}_{j}=\left(r_{j 1}, r_{j 2}, r_{j 3}\right)$ is the position of the $j$ th bead in space and $l_{0}$ is the mean distance between neighboring monomers.

For the E-P communication part, one may consider several forms of the potential. In the following, we introduce two most commonly used forms.

\section{Harmonic potential}

In the main text, the E-P communication is characterized by the harmonic spring, and the corresponding potential is assumed to take the form

$$
U_{\mathrm{EP}}\left(\boldsymbol{r}_{\mathrm{E}}, \boldsymbol{r}_{\mathrm{P}}\right)=\frac{1}{2} k_{\mathrm{EP}}\left(\left|\boldsymbol{r}_{\mathrm{E}}-\boldsymbol{r}_{\mathrm{P}}\right|-l_{0}\right)^{2},
$$

where $k_{\mathrm{EP}}$ is the spring coefficient between the enhancer and the promoter (also called 
communication strength), and $\mathrm{E}, \mathrm{P} \in\{1, \cdots, N\}$ represent the enhancer and the promoter with $\mathrm{E}<\mathrm{P}$. Let $d_{G}=\mathrm{P}-\mathrm{E}$ represents the E-P genomic distance (SI Fig. 1).

For analysis simplicity but without loss of generality, we set $l_{0}=0$ in Eq. (C2) and (C3). Since all the interactions we consider in the main text are represented by harmonic potentials, Eq. (C1) can be rewritten as

$$
\mathrm{d} \boldsymbol{r}=-\frac{1}{\gamma}\left[k_{\mathrm{NN}} \boldsymbol{M}_{\mathrm{NN}}+k_{\mathrm{EP}} \boldsymbol{M}_{\mathrm{EP}}\right] \boldsymbol{r} \mathrm{d} t+\sqrt{2 D} \mathrm{~d} \boldsymbol{B}(t),
$$

where $\boldsymbol{M}_{\mathrm{NN}}$ is a $N \times N$ matrix showing the connectivity between adjacent beads, given by

$$
\boldsymbol{M}_{\mathrm{NN}}=\left(\begin{array}{ccccccc}
1 & -1 & & & & & 0 \\
-1 & 2 & -1 & & & & \\
& -1 & 2 & \ddots & & & \\
& & \ddots & \ddots & \ddots & & \\
& & & \ddots & 2 & -1 & \\
& & & & -1 & 2 & -1 \\
0 & & & & & -1 & 1
\end{array}\right),
$$

and $\boldsymbol{M}_{\mathrm{EP}}$ is also a $N \times N$ matrix representing the E-P interaction, given by

$$
\boldsymbol{M}_{\mathrm{EP}}=\left(\begin{array}{ccccc} 
& \vdots & & \vdots & \\
\cdots & 1 & \cdots & -1 & \cdots \\
& \vdots & \ddots & \vdots & \\
\cdots & -1 & \cdots & 1 & \cdots \\
& \vdots & & \vdots &
\end{array}\right) \text { Eth row }
$$

Eq. (C4) with Eq. (C5) and (C6) characterize the movement of chromatin.

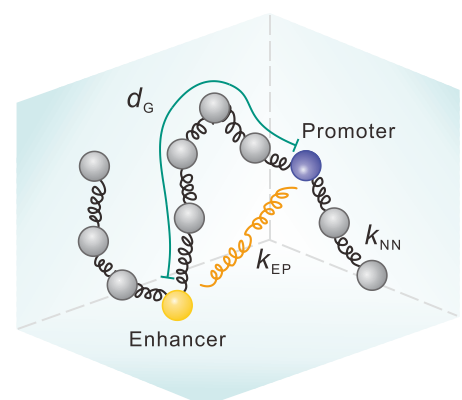

SI Figure 1. Schematic depiction of symbols related to chromatin structure.

\section{Lennard-Jones potential}

Besides the harmonic potential, the Lennard-Jones ( $\mathrm{LJ})$ potential is also commonly used in simulating molecular dynamics. Here, we modify the LJ potential function to fit our model. That is 


$$
U_{\mathrm{EP}}=4 \varepsilon\left[\left(\frac{\sigma}{\left\|\boldsymbol{r}_{\mathrm{E}}-\boldsymbol{r}_{\mathrm{P}}\right\|_{2}+s_{0}}\right)^{12}-\left(\frac{\sigma}{\left\|\boldsymbol{r}_{\mathrm{E}}-\boldsymbol{r}_{\mathrm{P}}\right\|_{2}+s_{0}}\right)^{6}\right],
$$

where $\sigma$ is a distance parameter, $\varepsilon$ is the depth of the potential well representing the strength of attraction between two particles, and $s_{0}$ is a modified distance. Based on Eq. (C7), derivatives $\nabla_{r_{\mathrm{E}}} U_{\mathrm{EP}}$ and $\nabla_{r_{\mathrm{P}}} U_{\mathrm{EP}}$ can be easily obtained. Then, Eq. (C1) can be rewritten as

$$
d \boldsymbol{r}_{i}=-\frac{1}{\gamma} \nabla_{\boldsymbol{r}_{i}} U_{\mathrm{NN}} d t-\frac{1}{\gamma} \nabla_{r_{i}} U_{\mathrm{EP}} d t+\sqrt{2 D} \mathrm{~d} \boldsymbol{B}_{i}(t), i=1,2, \cdots, N .
$$

Eq. (C8) describes chromatin motion. Note that the second term on the right hand side of Eq. (C8) is zero for $\boldsymbol{r}_{i}$ except $\boldsymbol{r}_{\mathrm{E}}$ and $\boldsymbol{r}_{\mathrm{p}}$. Apparently, Eq. (C8) that considers the LJ potential of complex form is different from Eq. (C4) that considers the potential of comparatively simpler form. In general, the former is more difficult to analyze and solve than the latter.

Finally, we point out that in subsection $\mathrm{C} 1$, communication strength $k_{\mathrm{EP}}$ depends, in general, on the inherent property of regulatory elements and the microenvironment around them. Different enhancers correspond to different communication strengths, so E-P communication strength is gene-specific. The E-P communication activating transcription (i.e., enhancerdependent transcription activation) often requires the presence of multiple TFs, such as general TFs and sequence-dependent effectors of signaling pathways, to ensure the integration of internal and external environmental cues via the E-P communication. Therefore, communication strength $k_{\mathrm{EP}}$ is taken as a key parameter of our model.

\section{C2 Multi-state model of downstream transcriptional bursting}

\section{Four-state model for transcription cycle}

In eukaryotic cells, mRNAs are usually produced in a burst mode that a high activity state follows a long refractory period. Such a biological phenomenon is frequently described by a telegraph model of gene switching, which consists of two states: an OFF state where the gene is not expressed and an ON state where the gene is transcribed. The two-state model provides a simple quantitative analysis framework, but transcription bursting is a complex biochemical process. Some gene-expression processes, e.g., those involving complex promoter (or E-P) dynamics, TFs and cofactors (COFs) dynamics and Pol II dynamics, would not be described by 
simple two-state models. Recent studies have extended two-state models to multistate models. In our main text, we used a four-state model to simulate transcriptional bursting dynamics with biological details stated below for better understanding.

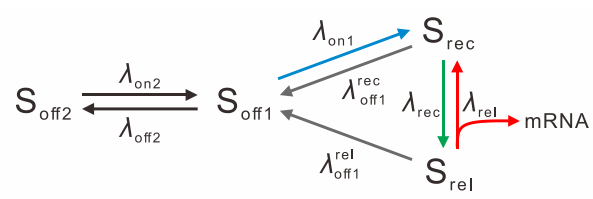

SI Figure 2. Kinetic scheme for a four-state model of transcriptional bursting.

OFF states - In the case that chromatin is silent, the mechanism of how a suitable transcriptional state for DNA transcription is created is unclear, but constantly increasing data have shown that the non-permissive period follows a non-exponential distribution $(13,46)$, indicating that the process from non-permissive to permissive transcription states is memorial, and is more complex rather than a single-step Markov process that is memoryless. This memory implies that a single OFF state in the traditional telegraph model is not enough to characterize the non-permissive state of chromatin. In addition, chromatin remodeling and nucleosome unwrapping due to the accessibility of chromatin are necessary for gene transcription. Pioneer TFs have unique properties that can open closed chromatin (47) to expose the promoter motif. Subsequently, the promoter, which is in a state of primed burst, employs general TFs, Mediator and Pol II for transcription. For this purpose, a deep inactive state ( $S_{\text {off }}$, closed chromatin) and a primed burst state $\left(\mathrm{S}_{\text {off }}\right)$ are considered as the $\mathrm{OFF}$ state $\left(\mathrm{S}_{\mathrm{OFF}}\right.$, non-permissive period).

ON states - Besides the non-permissive period (inactive state $\mathrm{S}_{\mathrm{OFF}}$ ), two most important processes in the transcription cycle are Pol II recruitment to the promoter and Pol II pause release from promoter-proximal $(9,48)$. Pol II is necessary for transcribing DNA into mRNA and paused Pol II is a common step in gene transcription and regulation $(49,50)$. Recent studies have indicated that paused Pol II is more stable than Pol II in the PIC stage $(38,51)$, and promoters with Pol II are more sensitive in response to stresses than those lacking Pol II (52). Meanwhile, paused Pol II can prevent new Pol II initiation to reduce transcriptional noise (38). After the release of paused Pol II, the next Pol II recruitment can be carried out. Since Pol II recruitment occurs after burst initiation (53), we employ Pol II recruitment state $\left(\mathrm{S}_{\mathrm{rec}}\right)$ and Pol II pause release state $\left(\mathrm{S}_{\mathrm{rel}}\right)$ as state $\mathrm{ON}\left(\mathrm{S}_{\mathrm{ON}}\right.$, permissive period) in the bursting stage. These 
two states, $S_{\text {rec }}$ and $S_{\text {rel }}$, can be switched to each other. And we assume that the process from $S_{\text {rel }}$ back to $S_{\text {rec }}$ generates an mRNA.

\section{State switching in four-state model}

The bursting system to be studied is described by a set of biochemical reactions on a slow timescale in contrast to the introduced-above system of chromatin motion on a fast timescale (see Section A and SI Fig. 2)

$$
\begin{aligned}
& \text { Chromatin opening: } S_{\text {off } 2} \stackrel{\lambda_{\text {on2 }}}{\longrightarrow} S_{\text {off1 }} \text {, } \\
& \text { Chromatin closure: } \quad S_{\text {off1 } 1} \stackrel{\lambda_{\text {of }}}{\longrightarrow} S_{\text {off } 2} \text {, }
\end{aligned}
$$

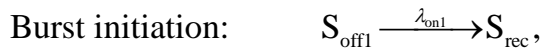

$$
\begin{aligned}
& \text { Pol II recruitment: } \quad \mathrm{S}_{\text {rec }} \stackrel{\lambda_{\text {rec }}}{\longrightarrow} S_{\text {rel }} \text {, } \\
& \text { Pol II pause release: } S_{\text {rel }} \stackrel{\lambda_{\text {rel }}}{\longrightarrow} S_{\text {rec }}+\text { mRNA, } \\
& \text { Burst termination1: } \quad S_{\text {rec }} \stackrel{\lambda_{\text {off }}^{\text {rec }} \longrightarrow}{\longrightarrow} S_{\text {offl }} \text {, } \\
& \text { Burst termination2: } \quad S_{\text {rel }} \stackrel{\lambda_{\text {oflt }}^{\text {rel }} \longrightarrow}{\longrightarrow} S_{\text {off1 }} \text {. }
\end{aligned}
$$

Note that rates $\lambda_{c}, c \in\{$ off2,off1,on2,on1,rec,rel $\}$ in Eq. (C9) are effective rates that summarize a series of sub-processes. Since burst termination is relatively dynamic (54), we hypothesize that both $\mathrm{S}_{\mathrm{rec}}$ and $\mathrm{S}_{\mathrm{rel}}$ states can return to $\mathrm{S}_{\mathrm{OFF}}$. Also because $\mathrm{S}_{\text {off }}$ is a deep inactive state, it is unlikely for $S_{\text {rec }}$ and $S_{\text {rel }}$ states to return directly to $S_{\text {off2 }}$ state in one step. Thus, we posit that only $S_{\text {off1 }}$ can be switched to $S_{\text {off2 }}$. Since the model has two burst termination channels, we add a superscript to distinguish these two termination rates ( $\lambda_{\text {offl }}^{\text {rec }}$ and $\left.\lambda_{\text {offl }}^{\text {rel }}\right)$. In order to help the readers' understanding and memory, we state the following simple facts. The toggle between states $S_{\text {off }}$ and $S_{\text {offl }}$ is regulated by the chromatin or nucleosome opening and rewrapping with rates $\lambda_{\text {on2 }}$ and $\lambda_{\text {off2 }}$, respectively. The transition from $S_{\text {off1 }}$ to $S_{\text {rec }}$ is associated with TFs binding with rate $\lambda_{\text {on }}$. The state switching from $S_{\text {rec }}$ to $S_{\text {rel }}$ is mediated by Pol II recruitment with rate $\lambda_{\text {rec }}$. The transition from $S_{\text {rel }}$ to $S_{\text {rec }}$ is linked to Pol II pause release with rate $\lambda_{\text {rel }}$ (which is also the rate of mRNA production). The transition from $S_{\text {rec }}$ to $S_{\text {off }}$ is related to the TFs unbinding, the disruption of TFs binding site or other processes with rate $\lambda_{\text {offl }}$, and the switching from $S_{\text {rel }}$ to $S_{\text {offl }}$ is involved in TFs and Pol II unbinding from the target gene, the collision of Pol II with exoribonucleases, or the disruption of Pol II active site (54) with rate $\lambda_{\text {off }}$. Note that before the gene transitions to state $\mathrm{S}_{\mathrm{OFF}}$, there would be many switches between $\mathrm{S}_{\text {rel }}$ and $\mathrm{S}_{\mathrm{rec}}$, thus producing bursts. 


\section{Transcriptional bursting process}

For the above biochemical reaction network, the characteristic of burst initiation is that $\mathrm{S}_{\text {off }}$ switches to $\mathrm{S}_{\mathrm{rec}}$ whereas that of the burst termination is that the $\mathrm{S}_{\mathrm{ON}}$ state returns back to the $\mathrm{S}_{\text {off } 1}$. If switching between $\mathrm{S}_{\text {off2 }}$ and $\mathrm{S}_{\text {off1 }}$ is relatively slow, this will result in periods of gene complete refractory, interspersed with sporadic bursty transcription.

Since the degradation of mRNA is not considered in the model, we assume that when the Pol II is released from the promoter-proximal pause, the transcription elongates along the genome for a fixed time $\tau_{\mathrm{E}}$, and when the Pol II reaches the end of the gene, the Pol II falls off and the nascent mRNA is no longer detected by snapshots.

Note that although our model is a multi-state one, it is different from multi-state models in previous studies $(55,56)$. Previous models assumed that gene state does not change when mRNA is produced (i.e., $\mathrm{ON} \rightarrow \mathrm{ON}+$ mRNA ), implying that the detailed processes of transcriptional bursting are ignored. In contrast, our model assumes, based on biological phenomena, that mRNA is produced in the process of switching from one state to another (i.e., $\mathrm{S}_{\mathrm{rel}} \rightarrow \mathrm{S}_{\mathrm{rec}}+\mathrm{mRNA}$ ). This is crucial for capturing the feature that only one Pol II is permitted to bind to promoter and the second Pol II recruitment must occur after the first Pol II pause release. Also, our model can capture some characteristics (such as traveling ratio, the effect of altering Pol II pause release rate) that cannot be obtained by the previous models.

\section{Transition probability matrix $W$}

In Eq. (C9), each state switching is regarded as a reaction process. However, in vivo, each state transition is generally not a single reaction but would be the synergism of multiple reactions (SI Table 1). Although there would be many sub-reactions behind each reaction, we suppose that the reaction events in the four-state model, i.e., Eq. (C9), are Markovian, i.e., the probabilities of reaction events only depend on the current state of the system, independent of the prior history (this hypothesis was made in almost previous theoretical studies). With this hypothesis, the waiting time of each reaction follows an exponential distribution. If a process occurs via multiple sub-reactions (or multiple steps) but only one of these steps is rate-limiting, then this process can be effectively described by one step. The number of rate-limiting steps of a reaction process is usually not large in gene transcription, so our Markov model can work 
well (15).

SI Table 1: Molecular processes of transcriptional bursting

\begin{tabular}{|c|c|c|c|}
\hline \multicolumn{2}{|c|}{ Gene state } & \multirow{2}{*}{$\begin{array}{l}\text { Reaction } \\
\mathrm{S}_{\text {off } 2} \rightarrow \mathrm{S}_{\text {off1 }}\end{array}$} & \multirow{2}{*}{$\begin{array}{l}\text { Biological processes } \\
\text { Pioneer TFs binding (57), opening chromatin ( } 58\end{array}$} \\
\hline \multirow[b]{2}{*}{ OFF } & $\begin{array}{l}\text { Deep inactive } \\
\text { state }\left(\mathrm{S}_{\text {off } 2}\right)\end{array}$ & & \\
\hline & $\begin{array}{l}\text { Primed burst } \\
\text { state }\left(\mathrm{S}_{\text {off } 1}\right)\end{array}$ & $\mathrm{S}_{\mathrm{off} 1} \rightarrow \mathrm{S}_{\mathrm{rec}}$ & $\begin{array}{l}\text { TFs recruitment (59), the binding of activators on } \\
\text { enhancer (60), the distortion of TATA box (35) and } \\
\text { alteration of chromatin structure (60). }\end{array}$ \\
\hline \multirow{4}{*}{$\mathrm{ON}$} & \multirow{2}{*}{$\begin{array}{l}\text { Pol II } \\
\text { recruitment } \\
\text { state }\left(S_{\text {rec }}\right)\end{array}$} & $\mathrm{S}_{\mathrm{rec}} \rightarrow \mathrm{S}_{\mathrm{offl}}$ & TFs unbinding, disruption of TFs binding site \\
\hline & & $\mathrm{S}_{\mathrm{rec}} \rightarrow \mathrm{S}_{\mathrm{rel}}$ & $\begin{array}{l}\text { Pol II recruitment (9), the formation of PIC (61), } \\
\text { promoter escape (62) and Pol II pausing (63). }\end{array}$ \\
\hline & \multirow{2}{*}{$\begin{array}{l}\text { Pol II pause } \\
\text { release state } \\
\left(S_{\text {rel }}\right)\end{array}$} & $\mathrm{S}_{\mathrm{rel}} \rightarrow \mathrm{S}_{\mathrm{offl}}$ & TFs and Pol II unbinding from the target gene. \\
\hline & & $\begin{array}{l}\mathrm{S}_{\mathrm{rel}} \rightarrow \mathrm{S}_{\mathrm{rec}} \\
+ \text { mRNA }\end{array}$ & $\begin{array}{l}\text { Pol II pause release (64), productive elongation } \\
\text { (65), Pol II termination (54) and another round of } \\
\text { transcription (9). }\end{array}$ \\
\hline
\end{tabular}

Based on the above description, the state transition matrix $\boldsymbol{W}(\boldsymbol{r} ; t)$ is expressed as

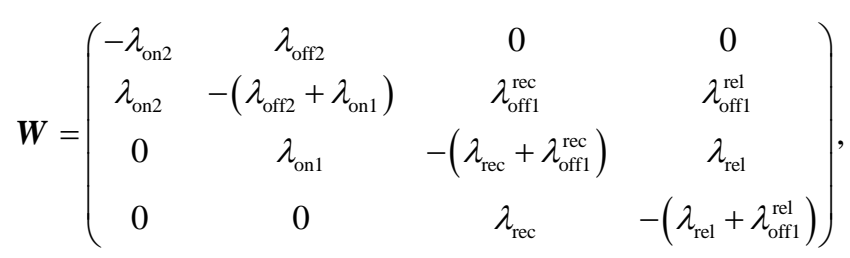

where all the $\lambda$-type parameters are dependent on the E-P spatial distance and will be discussed at Section C3.

\section{C3 Information flow from chromatin conformation to transcriptional bursting}

\section{Models of E-P communication}

Regulatory information from distal enhancers to target genes is needed to execute accurate transcription. Although various mechanisms have been proposed (such as loops and hubs), how E-P communication is carried out is still a matter of debate $(66,67)$. The looping model assumes E-P direct contact through molecular complexes. This assumption was often made in previous studies with a reason that looping can directly increase transcription levels and reactivate developmental genes $(40,68,69)$. However, in the work of Rao et al. (70) and Long et al. (71), active E-P pairs did not show an increased contact frequency, indicating that forming an E-P 
loop is not needed. Another viewpoint is the occurrence of transcription hubs, mainly because E-P interactions and the assembly of PIC facilitate that transcription loci forms clusters or hubs (66). Hypothetical hubs can well interpret the large spatial distance of E-P when transcription is activated. Therefore, the enhancer may not be too close to the target promoter. Recent experimental studies on measuring E-P spatial distances suggested a large distance on the order of a few hundred nanometers $(\mathrm{nm})(25,37)$. For example, the distance between the Sox 2 region and its essential enhancer in ESCs ranges from 200-400nm (72).

\section{Necessity of E-P proximity}

E-P proximity has been believed to increase the likelihood of transcription bursting. However, proximity does not mean direct contact. The distance between PP7 reporter gene and the enhancer decreases in transcriptional activation in contrast to the inactive state case, but the E-P distance in ON state is $\sim 340 \mathrm{~nm}$ (37), observably not via the direct contact distance. Throughout this paper, we use the hub hypothesis to investigate the transcription mechanism.

\section{Modeling information flow from chromatin structure to transcriptional bursting}

E-P communication, which plays a vital role in regulating stochastic gene expression, should be taken into account in transcriptional bursting. First, transcription activators binding to enhancers recruit TFs to alter chromatin structure and make it more accessible to active transcription (60). Second, E-P interaction co-regulates the recruitment of TFs and Pol II and the assembly of PIC $(73,74)$. Besides, enhancers recruit Mediator complex or histone acetyltransferase p300 to help the Pol II on promoters initiate transcription (75). Third, enhancers promote dissociation of NELF by recruiting COFs to affect Pol II pause-release on promoter-proximity (76). Correspondingly, gene state transition rates $\lambda_{c}$ of the biochemical reactions govern transcriptional bursting. According to the above experimental observations or evidences, we assume that the state transition rates are related to the E-P spatial distance denoted by $d_{\mathrm{S}}$. However, biological experiments did not tell us how these rates quantitatively depend on $d_{\mathrm{S}}$. In fact, this dependence relationship would be complex and in particular, it would be organism-specific. To simplify our analysis, we will, by making assumptions, set a special but common form of $\lambda_{c}$ to link the downstream transcription to the upstream chromatin configuration. 
Note that the opening chromatin (from $\mathrm{S}_{\text {off2 }}$ to $\mathrm{S}_{\text {offl }}$ ) would be mainly associated with pioneer TFs. Thus, we assume $\lambda_{\text {ofr }}$ is a constant independent of E-P spatial distance. Additionally, since the relationship between the $\lambda_{c}, c \in\{$ on1,rec,rel $\}$ and E-P distance may be opposite to that between $\lambda_{c}, c \in\{$ off1,off 2$\}$ and the distance, we set rates $\lambda_{\text {off } 2}, \lambda_{\text {off } 1}^{\text {rec }}$ and $\lambda_{\text {offl }}^{\text {rel }}$ as constants for simplicity.

First, chromatin structure generally does not act as a binary switch but acts as a modulator of gene function (non-binary) (1). Second, cooperative and synergistic binding to DNA is a common way in organisms. For example, in transcription processes, the binding of TFs may affect the binding rate of other TFs, Mediators or Pol II. Thus, it seems more reasonable to assume that the E-P spatial distance affects transcriptional burst rates in a nonlinear manner. Here we use Hill functions, which are very successful in modeling biological phenomena (77), to describe the transcription rates. To sum up, we assume that the piecewise continuous nonlinear rate function related to the E-P topological structure is

$$
\lambda_{c}\left(d_{\mathrm{S}}\right)=\left\{\begin{array}{cc}
\lambda_{c, \text { max }}, & d_{\mathrm{S}} \leq \varepsilon_{\mathrm{T}}, \\
\lambda_{c, \text { min }}+\left(\lambda_{c, \text { max }}-\lambda_{c, \text { min }}\right) /\left[1+\left(\frac{d_{\mathrm{S}}-\varepsilon_{\mathrm{T}}}{\varepsilon_{1 / 2}-\varepsilon_{\mathrm{T}}}\right)^{h}\right], & d_{\mathrm{S}}>\varepsilon_{\mathrm{T}},
\end{array}\right.
$$

where $\lambda_{c, \text { min }}$ and $\lambda_{c, \text { max }}$ are the minimum (basic) and maximum reaction rates for reactions $c \in\{$ on1,rec,rel $\}, \varepsilon_{1 / 2}$ is the spatial distance when $\lambda_{c}$ is equal to $\left(\lambda_{c, \text { max }}-\lambda_{c, \text { min }}\right) / 2, h$ is a Hill coefficient that controls how steep the rate curve is, and $\varepsilon_{\mathrm{T}}$ is a distance threshold (SI Fig. 3A and 3B). When the E-P distance is less than $\varepsilon_{\mathrm{T}}$, the $\varepsilon_{\mathrm{T}}$ is also used to describe the encounter of E-P. Therefore, it is reasonable to assume that the state transition rates are maximum when the distance is less than $\varepsilon_{\mathrm{T}}$ in Eq. (C11). Besides, encounter, which is merely an assumption in physical statistics, does not mean direct contact. As the distance increases, state transition rates can reduce to the minimum. Eq. (C11) can illustrate how the transition rates vary with E-P topologies, and further indicate that transcription is regulated at any time.

In our numerical simulation and theoretical analysis, we sufficiently use the relationship described by Eq. (C11). However, it is needed to point out that parameters $\lambda_{c}$, $c \in\{$ on1,rec,rel $\}$ may take other forms except for form (C11). In general, $\lambda_{c}$ may be set 
nonlinear decreasing functions of $d_{\mathrm{S}}$. Also, $\lambda_{c}$ can be set increasing functions to explain the special case that the enhancer activation of increasing E-P spatial distance $d_{\mathrm{S}}$ (78). Finally, $\lambda_{c}, c \in\{$ off1,off 2$\}$ may take a form of functions that are not constants.

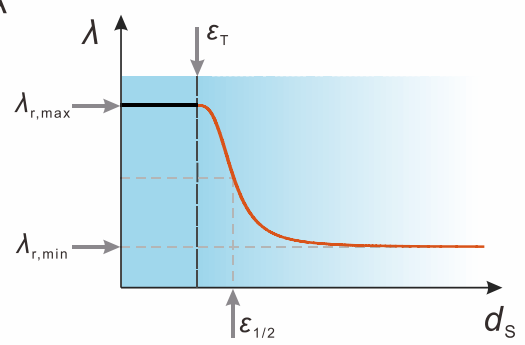

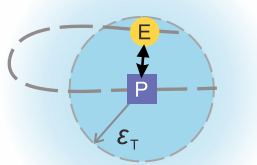

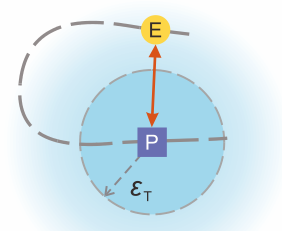

SI Figure 3. Schematic for the relationship between E-P spatial distance and gene state transition rates. (A) A piecewise nonbinary nonlinear Hill function describes the relationship between E-P spatial distance and burst rates. (B) E-P spatial distance is less (more) than $\varepsilon_{\mathrm{T}}$ in the left (right) panel. For the convenience of display, we take the promoter as the center position. 


\section{Simulation and statistical analysis of the whole model}

\section{Numerical simulations of the whole model}

The entire model, which considers both chromatin architecture and transcriptional bursting, is simulated using MATLAB (79). A numerical method has been described in the above Section B2. We examine a system consisting of $N=100$ monomers in simulations. To simplify, we consider a spatial region isolated from neighboring DNA by boundary insulator elements $(80$, 81). Biologically reasonable values of all the model parameters are listed in Section $\mathrm{H}$.

For a given set of parameters, we simulate $10^{3}$ gene copies, proceeding through $10^{7}$ seconds in time. Each simulation starts in the $S_{\text {off }}$ state. Before taking samples from every simulation, we run $10^{4}$ seconds to ensure equilibration between conformation and transcription. After that, snapshots of the system are taken every 100 seconds.

\section{Statistical analysis of simulation results}

Statistics analysis of upstream chromatin dynamics - Using the data obtained by simulation, we perform statistical analysis for the chromatin structure, especially for the E-P spatial distance. Specifically, we calculate the PDF of E-P spatial distance based on the produced time series data. Also, we calculate the E-P encounter probability (referring to SI Fig. 10E). Furthermore, we can alter the encounter distance to survey the E-P effective density or concentration. That is, we calculate the E-P encounter probability at some sphere with radius $R$ (E-P encounter distance is $2 R$ ), and then normalize this probability by the sphere volume as an effective density or concentration (referring to SI Fig. 10F, (82)).

Statistics analysis of downstream bursting kinetics - According to the reaction time series, we calculate the probability mass function (PMF) of burst size and the PDFs of the dwell time in $\mathrm{S}_{\mathrm{OFF}}$ and $\mathrm{S}_{\mathrm{ON}}$ states and the cycle time (the time from $\mathrm{S}_{\mathrm{OFF}}$ to $\mathrm{S}_{\mathrm{ON}}$ and back to $\mathrm{S}_{\mathrm{OFF}}$, referring to SI Figs. 13E-H). Furthermore, we calculate some statistical quantities such as mean burst size (MBS), mean dwell-time (MDT) in ON/OFF state, mean cycle time (MCT), burst frequency (BF). Here MBS is defined as the average number of mRNA molecules produced per burst whereas BF as the average number of bursts occurred in a given time interval. In other words, $\mathrm{BF}$ is the reciprocal of the MCT. In addition, our model can also be used to compute the mean traveling ratio (MTR), which is defined as the ratio of Pol II density in the gene body 
divided by the Pol II density at promoter proximity. Based on the data produced by simulation and by dividing the Pol II into two types upon its location: Pol II at promoter-proximal (5' end of the strand, Pol II at $S_{\text {rel }}$ state) and Pol II in the gene body (3' end of strand, a fixed time interval after Pol II pause release back to recruitment state, SI Fig. 4B), we calculate the MTR by uniformly-spaced sampling data (referring SI Fig. 19). 


\section{E Theoretical analysis of transcriptional bursting}

\section{E1 Timescale separation method decoupling upstream and downstream kinetics}

Note that burst size, dwell time and cycle time are random variables, denoted by $B S, D T$ and $C T$, respectively. We mainly calculate the PDFs or PMF of these random variables (SI Fig. 4) and their statistical quantities such as expectations. Also note that these variables are related to the state switching rates that however are linked to the E-P spatial distance due to the fact that the burst rates are regulated by E-P spatial distance (Section C3).

A

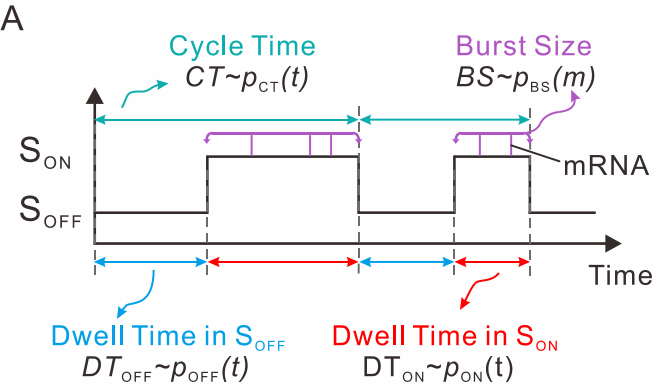

B

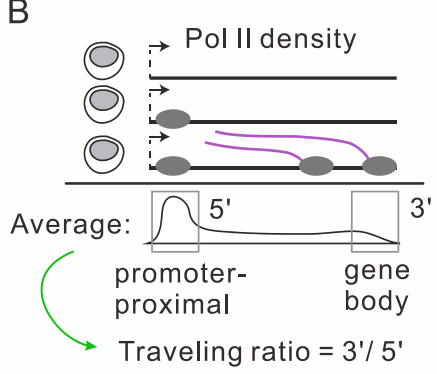

SI Figure 4. Meanings of symbols related to downstream transcriptional bursting. (A)

Schematic diagram of burst size, dwell time in ON/OFF state and cycle time. (B) Schematic diagram of traveling ratio.

\section{Timescale separation parameter}

Since the biochemical reactions for downstream transcriptional bursting occur on a slow timescale whereas the upstream chromatin motion takes place on a fast timescale, we can use a timescale separation method to carry out a theoretical analysis of bursting kinetics, although the timescale gap between the upstream and the downstream may be different in different cells or during different periods in the same cell. This method has been successfully used in the analysis of gene regulatory networks $(83,84)$ and the mutual information that disentangles interactions from changing environments (85). To quantify the timescale separation, we introduce a parameter $\omega$, which is defined as the ratio between the propensities of the reactions on fast and slow timescales

$$
\omega=\frac{\min \lambda_{c, \min }}{\max V(\boldsymbol{r})},
$$


where $\lambda_{c, \text { min }}, c \in\{$ on1,rec,rel $\}$ are the gene state switching rates in downstream transcription processes and $V(\boldsymbol{r})$ is the velocity field between the enhancer and the promoter in the upstream. In the following, we give detailed explanations.

\section{Upstream chromatin dynamics}

The random motion of chromatin leads to fluctuations in the E-P spatial distance $\left(d_{\mathrm{S}}\right)$. Let $D S$ be the random variable representing the E-P spatial distance. In principle, $D S$ should follows a distribution, i.e., $d_{\mathrm{S}} \sim p_{D S}\left(d_{\mathrm{S}}\right)$. In Section E2, we will derive the analytical expression of E-P spatial distance distribution $p_{D S}\left(d_{\mathrm{S}}\right)$ (referring to SI Figs. 10A and 10C).

In Eq. (E1), the E-P velocity field equals $V(r)=\frac{K_{\mathrm{EP}} d_{\mathrm{S}}}{\gamma}$, where $K_{\mathrm{EP}}$ represents the total spring coefficient between the enhancer and the promoter (see Section E2). We choose the value of $d_{\mathrm{S}}$ when cumulative density function $(\mathrm{CDF})$ of $p_{D S}\left(d_{\mathrm{S}}\right)$ reaches 0.99 , implying that the $d_{\mathrm{S}}$ reaches the maximum. This setting that corresponds to the maximum velocity is to include all possible cases of E-P spatial distance in simulations.

\section{Downstream transcriptional bursting}

For downstream random variables $X \in\{B S, D T \mid S, C T\}$, we calculate the joint distribution $p_{X, D S}\left(x, d_{\mathrm{S}}\right)$, which accounts for fluctuations in $d_{\mathrm{S}}$ and their effect on fluctuations in burst.

In the case that the fluctuations in $d_{\mathrm{S}}$ are much faster compared with the rate of transcription, the $d_{\mathrm{S}}$ fluctuations can be averaged out, and the corresponding joint distribution denoted by $p_{X, D S}^{\mathrm{Fast}}\left(x, d_{\mathrm{S}}\right)$ is given by

$$
p_{X, D S}^{\mathrm{Fast}}\left(x, d_{\mathrm{S}}\right)=p_{X}\left(x ;\left\langle\lambda_{c}\right\rangle\right) p_{D S}\left(d_{\mathrm{S}}\right),
$$

where $\left\langle\lambda_{c}\right\rangle=\int_{0}^{+\infty} \lambda_{c}\left(d_{\mathrm{S}}\right) p_{D S}\left(d_{\mathrm{S}}\right) \mathrm{d} d_{\mathrm{S}}$. In this case, the marginal PDF (or PMF) of random variable $X$ takes the form (referring to SI Figs. 12C-D)

$$
p_{X}^{\text {Fast }}(x)=p_{X \mid\langle D S\rangle}\left(x \mid \int_{0}^{+\infty} \lambda_{c}\left(d_{\mathrm{S}}\right) p_{D S}\left(d_{\mathrm{S}}\right) \mathrm{d} d_{\mathrm{S}}\right) .
$$

On the contrary, if fluctuations in $d_{\mathrm{s}}$ are much slower, the corresponding joint distribution denoted by $p_{X, D S}^{\text {Slow }}\left(x, d_{\mathrm{S}}\right)$ is given by 


$$
p_{X, D S}^{\text {Slow }}\left(x, d_{\mathrm{S}}\right)=p_{X \mid D S}\left(x \mid \lambda_{c}\left(d_{\mathrm{S}}\right)\right) p_{D S}\left(d_{\mathrm{S}}\right) .
$$

In this situation, the marginal PDF (or PMF) of random variable $X$ takes the form (referring to SI Figs. 12E-F)

$$
p_{X}^{\text {Slow }}(x)=\int_{0}^{+\infty} p_{X \mid D S}\left(x \mid \lambda_{c}\left(d_{\mathrm{S}}\right)\right) p_{D S}\left(d_{\mathrm{S}}\right) \mathrm{d} d_{\mathrm{S}} .
$$

In general, the marginal PDF (or PMF) of $X \in\{B S, D T \mid S, C T\}$ is a mixing distribution obtained by weighting the marginal PDF (or PMF) given in Eq. (E3) and Eq. (E5) (referring to SI Figs. 13A-D)

$$
p_{X}(x)=\frac{1}{1+\omega} p_{X}^{\text {Fast }}(x)+\frac{\omega}{1+\omega} p_{X}^{\text {Slow }}(x) .
$$

Based on Eq. (E6), the PMF of burst size $p_{B S}(m)$, the PDF of dwell time $p_{S}(t)$ (note: the complete expression is $p_{D T \mid S}(t \mid s)$ where $s \in\{$ off2,off1,rec,rel $\}$ or $\left.s \in\{\mathrm{OFF}, \mathrm{ON}\}\right)$ and the PDF of cycle time $p_{C T}(t)$ can be obtained (SI Fig. 4A). In particular, the expectation of $X$ can be expressed as

$$
\langle X\rangle=\frac{1}{1+\omega}\langle X\rangle^{\text {Fast }}+\frac{\omega}{1+\omega}\langle X\rangle^{\text {Slow }}
$$

Finally, we point out that since the traveling ratio is the ratio of two quantities as defined in Section $\mathrm{D}$, expectation $E[T R]$ can be directly derived, where $T R$ is a random variable representing traveling ratio (SI Fig. 4B).

In Section E3, we will derive the analytical expressions of the burst size, dwell time, cycle time and traveling ratio when we only consider downstream transcriptional bursts. Note that the PDF or PMF obtained in Section E3 is actually the cPDF. Then, the PDF or PMF of the whole transcriptional bursting can be obtained by Eq. (E6).

\section{E2 Analytical results for upstream chromatin dynamics}

In this subsection, we derive the explicit expression of the E-P spatial distance distribution. Term $k_{\mathrm{NN}} \boldsymbol{M}_{\mathrm{NN}}+k_{\mathrm{EP}} \boldsymbol{M}_{\mathrm{EP}}$ in Eq. (C4) is a singular matrix. To eliminate the degrees of freedom, the position of the first monomer in the chain can be set as $\boldsymbol{r}_{1} \equiv \mathbf{0}$ based on the methods in (86). Thus, Eq. (B5) can be rewritten as 


$$
\frac{\partial p(\tilde{\boldsymbol{r}} ; t)}{\partial t}=\nabla_{\tilde{\boldsymbol{r}}} \cdot\left(\frac{1}{\gamma}\left[k_{\mathrm{NN}} \tilde{\boldsymbol{M}}_{\mathrm{NN}}+k_{\mathrm{EP}} \tilde{\boldsymbol{M}}_{\mathrm{EP}}\right] \tilde{\boldsymbol{r}} p(\tilde{\boldsymbol{r}} ; t)\right)+\nabla_{\tilde{\boldsymbol{r}}}^{2}(D p(\tilde{\boldsymbol{r}} ; t)),
$$

where $\tilde{\boldsymbol{r}}=\left(r_{i j}\right)_{(N-1) \times 3}=\left[\boldsymbol{r}_{2}, \cdots, \boldsymbol{r}_{N}\right]^{\mathrm{T}}$, and

$$
\tilde{\boldsymbol{M}}_{\mathrm{NN}}=\left(\begin{array}{ccccc}
2 & -1 & & & 0 \\
-1 & 2 & \ddots & & \\
& \ddots & \ddots & \ddots & \\
& & \ddots & 2 & -1 \\
0 & & & -1 & 1
\end{array}\right), \tilde{\boldsymbol{M}}_{\mathrm{EP}}=\left(\begin{array}{ccccc} 
& \vdots & & \vdots \\
\cdots & 1 & \cdots & -1 & \cdots \\
& \vdots & & \vdots & \\
\cdots & -1 & \cdots & 1 & \cdots \\
& \vdots & & \vdots &
\end{array}\right)(\mathrm{P}-1) \text { th row }
$$

where $.1<\mathrm{E}<\mathrm{P}$..

Owing to the fact that every monomer moves independently in each dimension, the PDF for chromatin conformation $\tilde{\boldsymbol{r}}$ can be expressed as

$$
p\left(\boldsymbol{r}_{2}, \cdots, \boldsymbol{r}_{N}\right)=\prod_{i=1}^{3} p_{i}\left(r_{2 i}, \cdots, r_{N i}\right)
$$

In fact, the monomer motion in Eq. (E8) is an Ornstein-Uhlenbeck process and the general solution to this equation is a Gaussian distribution. If we consider one-dimensional PDF $p_{i}$, the $p_{i}$ can be analytically expressed as (87)

$$
p_{i}\left(r_{2 i}, \cdots, r_{N i}\right)=C \exp \left(-\frac{1}{2}\left(r_{2 i}, \cdots, r_{N i}\right)^{\mathrm{T}} \boldsymbol{\Sigma}^{-1}\left(r_{2 i}, \cdots, r_{N i}\right)\right),
$$

where $C$ is a normalization constant and

$$
\Sigma_{(N-1) \times(N-1)}^{-1}=\frac{1}{D \gamma}\left(k_{\mathrm{NN}} \tilde{\boldsymbol{M}}_{\mathrm{NN}}+k_{\mathrm{EP}} \tilde{\boldsymbol{M}}_{\mathrm{EP}}\right) .
$$

The $p_{i}$ is a multivariate normal distribution with the zero mean and the covariance matrix being $\boldsymbol{\Sigma}$. The marginal distributions for the enhancer and promoter are calculated according to

$$
\begin{aligned}
& p_{i}\left(r_{\mathrm{E} i}\right)=\int \cdots \int p_{i}\left(r_{2 i}, \cdots, r_{N i}\right) \prod_{\substack{l \neq 2, l \neq \mathrm{E}}}^{N} \mathrm{~d} r_{l i}, \\
& p_{i}\left(r_{\mathrm{P} i}\right)=\int \cdots \int p_{i}\left(r_{2 i}, \cdots, r_{N i}\right) \prod_{\substack{l=2, l \neq \mathrm{P}}}^{N} \mathrm{~d} r_{l i} .
\end{aligned}
$$

Based on the properties of Gaussian distribution, the marginal PDFs in Eq. (E13) are also Gaussian distributions.

By tedious calculating, we find that the analytical expression of the PDF $p_{D S}\left(d_{\mathrm{S}}\right)$ of the E-P spatial distance takes the form

$$
p_{D S}\left(d_{\mathrm{S}}\right)=\sqrt{\frac{2}{\pi}} \Theta^{-3} d_{\mathrm{S}}^{2} \exp \left(-\frac{d_{\mathrm{S}}^{2}}{2 \Theta^{2}}\right)
$$


where

$$
\Theta=\sqrt{D \gamma\left(\frac{k_{\mathrm{NN}}}{d_{\mathrm{G}}}+k_{\mathrm{EP}}\right)^{-1}}
$$

In Eq. (E15), $d_{\mathrm{G}}$ is the E-P genomic distance, i.e., $d_{G}=\mathrm{P}-\mathrm{E}$, which is different from the EP spatial distance $d_{\mathrm{S}}$ (SI Fig. 1). Note that Eq. (E14) is a Maxwell-Boltzmann distribution (referring to SI Fig. 10A), which can be regarded as the positive square root of the sum of squares of three independent random variables with each following the same normal distribution. Equivalently, each normal distribution represents the E-P distance in a certain dimension. Thus, Eq. (E14) is the distribution of the E-P Euclidean distance in 3D. More precisely, Eq. (E14) is equivalent to the chi distribution with three degrees of freedom and a scale parameter $\Theta$.

Except for the harmonic spring $k_{\mathrm{EP}}$ between the enhancer and the promoter in Eq. (E15), which accounts for the E-P communication, the enhancer and the promoter are also connected in series by $d_{\mathrm{G}}$ springs with the same elastic coefficient $k_{\mathrm{NN}}$. These connected springs are in effect equivalent to a spring with an elastic coefficient $k_{\mathrm{NN}} / d_{\mathrm{G}}$. Thus, the connection between the enhancer and the promoter can be viewed as two paralleling springs, which are further equivalent to a spring with an elastic coefficient $\frac{k_{\mathrm{NN}}}{d_{\mathrm{G}}}+k_{\mathrm{EP}}$. Due to the Einstein relation $D \gamma=k_{B} T$, the distribution is determined by the temperature $T$ (or the product of $D$ and $\gamma$, rather than $D$ or $\gamma$ alone) and the equivalent spring. Furthermore, the scale parameter $\Theta$ in Eq. (E15) measures the spatial distance in units proportional to the square root of the ratio of temperature $T$ and spring coefficient $\frac{k_{\mathrm{NN}}}{d_{\mathrm{G}}}+k_{\mathrm{EP}}$. In Eq. (E1), $V(\boldsymbol{r})=\frac{1}{\gamma}\left(\frac{k_{\mathrm{NN}}}{d_{\mathrm{G}}}+k_{\mathrm{EP}}\right) d_{\mathrm{S}}$.

Here we give details for deriving Eq. (E15). Making use of the fact that every monomer moves independently in each direction in 3-dimensional space, we can show that the lumping parameter $\Theta$ is expressed as $\Theta=\sqrt{\sum_{\mathrm{E}-1, \mathrm{E}-1}+\Sigma_{\mathrm{P}-1, \mathrm{P}-1}-2 \Sigma_{\mathrm{E}-1, \mathrm{P}-1}}$ where $\Sigma_{i, j}$ are the elements of matrix $\Sigma_{(N-1) \times(N-1)}$. For convenience, we define $\tilde{\boldsymbol{M}}_{\mathrm{Rouse}} \triangleq \boldsymbol{Q}$ and $\frac{k_{\mathrm{EP}}}{k_{\mathrm{NN}}} \tilde{\boldsymbol{M}}_{\mathrm{EP}} \triangleq \boldsymbol{R}$. Then, Eq. (E12) can be rewritten as

$$
\boldsymbol{\Sigma}_{(N-1) \times(N-1)}^{-1}=\frac{k_{\mathrm{NN}}}{D \gamma}(\boldsymbol{Q}+\boldsymbol{R})
$$


Obviously, the matrices $\boldsymbol{Q}$ and $\boldsymbol{Q}+\boldsymbol{R}$ are nonsingular matrices, and $\boldsymbol{R}$ is a matrix of rank one. Thus, the inverse of $\boldsymbol{Q}+\boldsymbol{R}$ is (88)

$$
(\boldsymbol{Q}+\boldsymbol{R})^{-1}=\boldsymbol{Q}^{-1}-\frac{1}{1+\operatorname{tr}\left(\boldsymbol{R} \boldsymbol{Q}^{-1}\right)} \boldsymbol{Q}^{-1} \boldsymbol{R} \boldsymbol{Q}^{-1}
$$

Because $\boldsymbol{Q}$ is a tridiagonal matrix, its inverse is given by (89)

$$
\left(\boldsymbol{Q}^{-1}\right)_{i j}= \begin{cases}(-1)^{i+j}(-1)^{j-i} \frac{\rho_{i-1} \phi_{j+1}}{\rho_{N-1}}, & i \leq j, \\ (-1)^{i+j}(-1)^{i-j} \frac{\rho_{j-1} \phi_{i+1}}{\rho_{N-1}}, & i>j,\end{cases}
$$

where $\rho_{k}=k+1(k=0,1, \cdots, N-2), \quad \rho_{N-1}=\operatorname{det}(\boldsymbol{Q})=1, \quad \phi_{k}=1(k=1,2, \cdots, N)$. Then

$$
\left(Q^{-1}\right)_{i j}=\left\{\begin{array}{ll}
\rho_{i-1} \phi_{j+1} / \rho_{N-1}, & i \leq j, \\
\rho_{j-1} \phi_{i+1} / \rho_{N-1}, & i>j,
\end{array}=\left\{\begin{array}{ll}
i, & i \leq j \\
j, & i>j,
\end{array}=\min (i, j) .\right.\right.
$$

Hence,

$$
\boldsymbol{R} \boldsymbol{Q}^{-1}=\frac{k_{\mathrm{EP}}}{k_{\mathrm{NN}}}\left[\cdots, \underset{(\mathrm{E}-1) \text { th column }}{-\boldsymbol{\alpha}^{\mathrm{T}},} \cdots, \underset{(\mathrm{P}-1) \text { th column }}{\boldsymbol{\alpha}^{\mathrm{T}},} \cdots\right]_{(N-1) \times(N-1)}^{\mathrm{T}},
$$

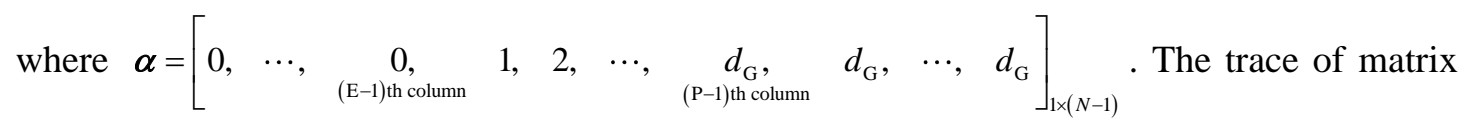
$\boldsymbol{R} \boldsymbol{Q}^{-1}$ is given by

$$
\operatorname{tr}\left(\boldsymbol{R} \boldsymbol{Q}^{-1}\right)=\frac{k_{\mathrm{EP}}}{k_{\mathrm{NN}}} d_{\mathrm{G}}
$$

We then have

$$
\boldsymbol{Q}^{-1} \boldsymbol{R} \boldsymbol{Q}^{-1}=\frac{k_{\mathrm{EP}}}{k_{\mathrm{NN}}} \boldsymbol{\alpha}^{\mathrm{T}} \boldsymbol{\alpha}
$$

Therefore, based on Eq. (E16) - (E22), we can calculate matrix $\boldsymbol{\Sigma}_{(N-1) \times(N-1)}$. However, it is no need to calculate all the elements of $\boldsymbol{\Sigma}_{(N-1) \times(N-1)}$. Eq. (E15) can be thus derived.

Finally, we would like to point out: (1) the statement that $d_{\mathrm{G}}$ is the E-P genomic distance is not accurate, but $d_{\mathrm{G}}$ is actually the number of monomers between the enhancer and the promoter in simulation. If one monomer is assumed to be a nucleosome, the E-P genomic distance should be expressed as $200 d_{\mathrm{G}} \mathrm{bp}$, where $200 \mathrm{bp}$ is the DNA length around the nucleosome; (2) the total genomic length is independent of the E-P spatial distance distribution 
$p_{D S}\left(d_{\mathrm{S}}\right)$. In numerical simulations, the number of simulated monomers is thus unimportant compared with that of monomers between the enhancer and the promoter, i.e., $d_{\mathrm{G}} ;(3)$ the form of modified LJ potential between the enhancer and the promoter in Eq. (C7) merely provides a strategy that replaces the harmonic spring potential. Although the LJ potential is more difficult to analyze, numerical simulations find that LJ potential can be also fitted with MaxwellBoltzmann distribution (referring to SI Fig. 10G).

\section{E3 Analytical results for downstream bursting kinetics}

In this subsection, we consider the downstream transcriptional bursting. The PDF or PMF obtained in this subsection is actually the cPDF given the E-P spatial distance $d_{\mathrm{s}}$.

For our four-state model, an equivalent model with an absorbing boundary state $S_{a b s}$ is shown in SI Fig. 5. The following analysis is mainly based on SI Fig. 5. Burst size is unrelated to the state $\mathrm{S}_{\mathrm{OFF}}$ since only when the gene is activated $\left(\mathrm{S}_{\mathrm{ON}}\right)$ can mRNA be produced. Besides, the residence time of the state $\mathrm{S}_{\mathrm{OFF}}$ can be calculated independently from other states due to the particularity of $\mathrm{S}_{\mathrm{OFF}}$. Thus, we first focus on the burst stage (i.e., $\mathrm{S}_{\mathrm{ON}}$ ) and ignore the state $\mathrm{S}_{\mathrm{OFF}}$ in the following derivation. Let the initial time of burst stage $(t=0)$ be set at the moment when the state $S_{\text {off1 }}$ switches to the state $S_{\text {rec }}$.

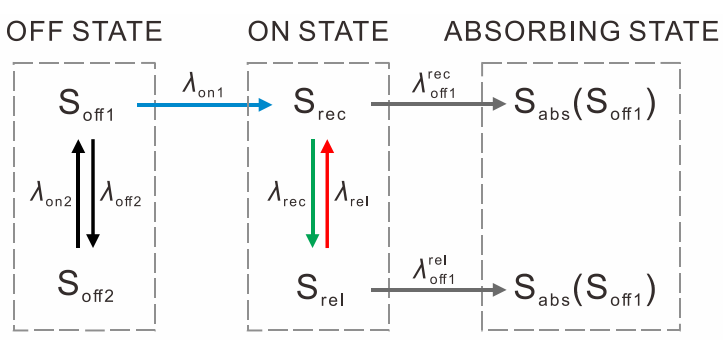

SI Figure 5. Kinetic scheme of an equivalent four-state model of transcriptional bursting.

Let $H_{T \mid B S, S}(t \mid m, s)$ be the survival probability that $m$ mRNAs are produced during a burst (i.e., $B S=m, m=0,1,2, \cdots)$ in the state $S$ (i.e., $S=s, s \in\{$ rec, rel $\}$ ) at the time $t$ (i.e., $T=t$ ). Then,

$$
H_{T \mid B S, S}(t \mid m, s)=\operatorname{Pr}\left(T_{\text {exit }}>t \mid B S=m, S=s\right),
$$


where $T_{\text {exit }}$ is the exit time from the state $s$. Hereafter we define $H_{T \mid B S, S}(t \mid-1, s) \equiv 0$. Note that the expressions of the state $s \in\{$ rec, rel $\}$ in formulas and of the state $\mathrm{S}_{\mathrm{rec}}, \mathrm{S}_{\mathrm{rel}}$ in the main text may be different but their meanings are the same. In the following, we rewrite the survival probability $H_{T \mid B S, S}(t \mid m, s)$ as $H_{m, s}(t)$ for the sake of simplicity and beauty of the formulas.

Based on Eq. (E23), we consider an infinitesimal interval $(t, t+\Delta t)$. Then, $H_{m, s}(t+\Delta t)$ can be expressed as

$$
\begin{aligned}
& H_{m, \text { rec }}(t+\Delta t)=H_{m, \text { rec }}(t)-H_{m, \text { rec }}(t) \lambda_{\text {rec }} \Delta t+H_{m-1, \text { rel }}(t) \lambda_{\text {rel }} \Delta t-H_{m, \text { rec }}(t) \lambda_{\text {offl }}^{\text {rec }} \Delta t \\
& H_{m, \text { rel }}(t+\Delta t)=H_{m, \text { rel }}(t)+H_{m, \text { rec }}(t) \lambda_{\text {rec }} \Delta t-H_{m, \text { rel }}(t) \lambda_{\text {rel }} \Delta t-H_{m, \text { rel }}(t) \lambda_{\text {off } 1}^{\text {rel }} \Delta t
\end{aligned}
$$

The master equations determining the probabilities $H_{m, s}(t)$ from Eq. (E24) are

$$
\begin{aligned}
& \frac{\mathrm{d} H_{m, \text { rec }}(t)}{\mathrm{d} t}=-\lambda_{\text {rec }} H_{m, \text { rec }}(t)+\lambda_{\text {rel }} H_{m-1, \text { rel }}(t)-\lambda_{\text {off }}^{\text {rec }} H_{m, \text { rec }}(t), \\
& \frac{\mathrm{d} H_{m, \text { rel }}(t)}{\mathrm{d} t}=\lambda_{\text {rec }} H_{m, \text { rec }}(t)-\lambda_{\text {rel }} H_{m, \text { rel }}(t)-\lambda_{\text {off } 1}^{\text {rel }} H_{m, \text { rel }}(t) .
\end{aligned}
$$

This equation group is valid for all $m \geq 0$.

To solve Eq. (E25), we introduce the generating function $G_{s}(z, t)$ for $H_{m, s}(t)$

$$
G_{\text {rec }}(z, t)=\sum_{m=0}^{\infty} H_{m, \text { rec }}(t) z^{m}, G_{\text {rel }}(z, t)=\sum_{m=0}^{\infty} H_{m, \text { rel }}(t) z^{m} .
$$

Then, we have the following partial differential equations

$$
\begin{aligned}
& \frac{\partial}{\partial t} G_{\text {rec }}(z, t)=-\left(\lambda_{\text {rec }}+\lambda_{\text {offl }}^{\text {rec }}\right) G_{\text {rec }}(z, t)+\lambda_{\text {rel }} z G_{\text {rel }}(z, t), \\
& \frac{\partial}{\partial t} G_{\text {rel }}(z, t)=\lambda_{\text {rec }} G_{\text {rec }}(z, t)-\left(\lambda_{\text {rel }}+\lambda_{\text {off }}^{\text {rec }}\right) G_{\text {rel }}(z, t) .
\end{aligned}
$$

Eliminating $G_{\text {rec }}(z, t)$ from Eq. (E27), we obtain the following second-order differential equation

$$
\begin{aligned}
& \frac{\partial^{2}}{\partial t^{2}} G_{\text {rel }}(z, t)+\left(\lambda_{\text {rec }}+\lambda_{\text {rel }}+\lambda_{\text {offl }}^{\text {rec }}+\lambda_{\text {offl }}^{\text {rel }}\right) \frac{\partial}{\partial t} G_{\text {rel }}(z, t) \\
& +\left[\left(\lambda_{\text {rel }}+\lambda_{\text {offl }}^{\text {rec }}\right)\left(\lambda_{\text {rec }}+\lambda_{\text {offl }}^{\text {rel }}\right)-\lambda_{\text {rec }} \lambda_{\text {rel }} z\right] G_{\text {rel }}(z, t)=0 .
\end{aligned}
$$

This equation can be viewed as an ordinary differential equation with constant coefficients. The corresponding characteristic equation is

$$
x^{2}+\left(\lambda_{\text {rec }}+\lambda_{\text {rel }}+\lambda_{\text {offl }}^{\text {rec }}+\lambda_{\text {off } 1}^{\text {rel }}\right) x+\left(\lambda_{\text {rel }}+\lambda_{\text {off } 1}^{\text {rec }}\right)\left(\lambda_{\text {rec }}+\lambda_{\text {offl }}^{\text {rel }}\right)-\lambda_{\text {rec }} \lambda_{\text {rel }} z=0 .
$$


Solving this algebraic equation yields

$$
x_{ \pm}(z)=\frac{1}{2}\left[-\left(\lambda_{\text {rec }}+\lambda_{\text {rel }}+\lambda_{\text {off } 1}^{\text {rec }}+\lambda_{\text {offl }}^{\text {rel }}\right) \pm \sqrt{\left(\left(\lambda_{\text {rec }}+\lambda_{\text {off }}^{\text {rec }}\right)-\left(\lambda_{\text {rel }}+\lambda_{\text {off } 1}^{\text {rel }}\right)\right)^{2}+4 \lambda_{\text {rec }} \lambda_{\text {rel }} z}\right]
$$

and we can show $x_{ \pm}<0$. Thus, a general solution to Eq. (E28) is

$$
G_{\text {rel }}(z, t)=C_{1} e^{x_{+}(z) t}+C_{2} e^{x_{-}(z) t},
$$

where $C_{1}$ and $C_{2}$ are constants determined by initial conditions. Substituting Eq. (E31) into the second equation of Eq. (E27), we have

$$
G_{\text {rec }}(z, t)=\frac{C_{1}}{\lambda_{\text {rec }}}\left(\lambda_{\text {rel }}+\lambda_{\text {off1 }}^{\text {rel }}+x_{+}(z)\right) e^{x_{+}(z) t}+\frac{C_{2}}{\lambda_{\text {rec }}}\left(\lambda_{\text {rel }}+\lambda_{\text {off1 }}^{\text {rel }}+x_{-}(z)\right) e^{x_{-}(z) t} .
$$

Note that $H_{0, \text { rec }}(0)$ is the initial state of a burst. If taking $H_{0, \text { rec }}(0)=1, H_{0, \text { rel }}(0)=0$ and $H_{m, s}(0)=0 \quad(s \in\{\mathrm{rec}, \mathrm{rel}\}, m=1,2, \cdots)$ as the initial conditions, we have the following algebraic equation group determining two constants $C_{1}$ and $C_{2}$

$$
\begin{aligned}
& G_{\text {rec }}(z, 0)=\frac{C_{1}}{\lambda_{\text {rec }}}\left(\lambda_{\text {rel }}+\lambda_{\text {off } 1}^{\text {rel }}+x_{+}(z)\right)+\frac{C_{2}}{\lambda_{\text {rec }}}\left(\lambda_{\text {rel }}+\lambda_{\text {off } 1}^{\text {rel }}+x_{-}(z)\right)=1, \\
& G_{\text {rel }}(z, 0)=C_{1}+C_{2}=0 .
\end{aligned}
$$

Solving Eq. (E33) gives

$$
C_{1}=\frac{\lambda_{\mathrm{rec}}}{x_{+}(z)-x_{-}(z)}, C_{2}=-\frac{\lambda_{\mathrm{rec}}}{x_{+}(z)-x_{-}(z)} .
$$

Substituting Eq. (E34) into Eq. (E31) and (E32), we know that the solution of Eq. (E27) is

$$
\begin{aligned}
G_{\text {rec }}(z, t) & =\frac{1}{x_{+}(z)-x_{-}(z)}\left[\left(\lambda_{\text {rel }}+\lambda_{\text {offl }}^{\text {rel }}+x_{+}\right) e^{x_{+}(z) t}-\left(\lambda_{\text {rel }}+\lambda_{\text {off } 1}^{\text {rel }}+x_{-}\right) e^{x_{-}(z) t}\right], \\
G_{\text {rel }}(z, t) & =\frac{\lambda_{\text {rec }}}{x_{+}(z)-x_{-}(z)}\left(e^{x_{+}(z) t}-e^{x_{-}(z) t}\right) .
\end{aligned}
$$

To that end, we have obtained the solution of the generating function corresponding to the survival probability.

\section{Burst size}

In our four-state model, both $\mathrm{S}_{\mathrm{rec}}$ and $\mathrm{S}_{\text {rel }}$ can return to $\mathrm{S}_{\text {offl }}$ so that the burst ends. And the transitions between $S_{\text {rec }}$ and $S_{\text {rel }}$ can be carried out many times before one burst ends, resulting in the bursty production of mRNAs.

The probability that burst termination time $T_{\text {term }}$ falls within an infinitesimal interval $(t, t+\Delta t)$ equals 


$$
\mathrm{P}\left(t<T_{\text {term }}<t+\Delta t \mid T_{\text {term }}>t, X=m\right)=\left(\lambda_{\text {offl }}^{\text {rec }} H_{m, \text { rec }}(t)+\lambda_{\text {off } 1}^{\text {rel }} H_{m, \text { rel }}(t)\right) \Delta t .
$$

The joint probability density $p_{B S, T_{\text {term }}}(m, t)$ for $B S$ (discrete random variable) and burst termination time $T_{\text {term }}$ (continuous random variable) include all incoming fluxes driving the system from state $\mathrm{S}_{\text {rec }}$ and $\mathrm{S}_{\text {rel }}$ to state $\mathrm{S}_{\mathrm{abs}}$ (or $\mathrm{S}_{\text {off }}$ ) (SI Fig. 6). That is,

$$
p_{B S, T_{\text {tem }}}(m, t)=\lambda_{\text {offl }}^{\text {rec }} H_{m, \text { rec }}(t)+\lambda_{\text {offl }}^{\text {rel }} H_{m, \text { rel }}(t)
$$

Then, the marginal probability distribution for BS is calculated according to

$$
\begin{aligned}
& p_{B S}(m)=\int_{0}^{\infty} p_{B S, T_{\text {term }}}(m, t) \mathrm{d} t=\int_{0}^{\infty}\left(\lambda_{\text {offl }}^{\text {rec }} H_{m, \text { rec }}(t)+\lambda_{\text {offl }}^{\text {rel }} H_{m, \text { rel }}(t)\right) \mathrm{d} t .
\end{aligned}
$$

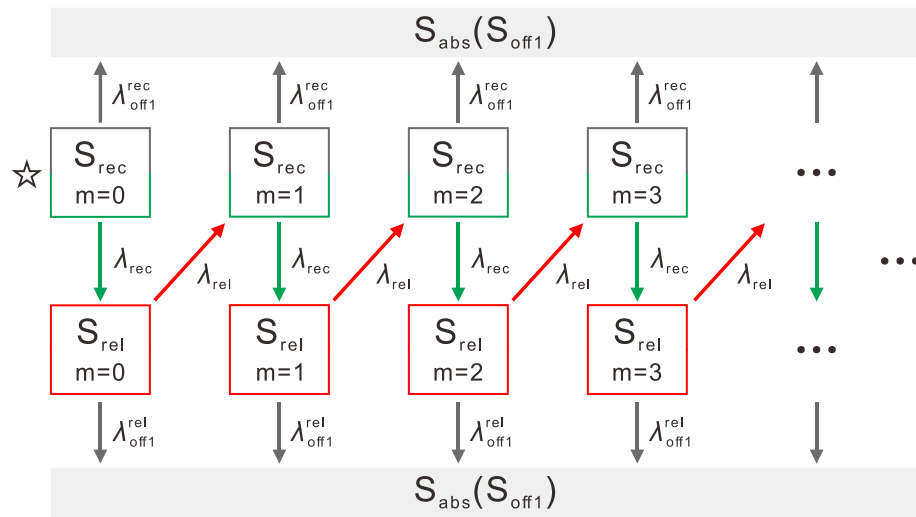

SI Figure 6. Schematic for the transitions between discrete states and the generation of mRNAs. $\mathrm{S}_{\mathrm{rec}}, \mathrm{S}_{\mathrm{rel}}$ and $\mathrm{S}_{\mathrm{abs}}$ are three states in the model. $m=0,1,2, \cdots$ is the number of mRNAs. $\lambda_{c}, c \in\{$ rec, rel, off 1$\}$ are burst rates. The star in the left top corner is the initial state of a burst.

Denote by $G(z)$ the generating function for $p_{B S}(m)$. Then based on Eq. (E35) and Eq. (E38), we can obtain

$$
\begin{aligned}
G(z) & =\int_{0}^{\infty} \lambda_{\text {off } 1}^{\text {rec }} G_{\text {rec }}(z, t) \mathrm{d} t+\int_{0}^{\infty} \lambda_{\text {off1 }}^{\text {rel }} G_{\text {rel }}(z, t) \mathrm{d} t \\
& =\frac{\lambda_{\text {rec }} \lambda_{\text {off } 1}^{\text {rel }}+\left(\lambda_{\text {rel }}+\lambda_{\text {off } 1}^{\text {rel }}\right) \lambda_{\text {off1 }}^{\text {rec }}}{\left(\lambda_{\text {rec }}+\lambda_{\text {off } 1}^{\text {rec }}\right)\left(\lambda_{\text {rel }}+\lambda_{\text {off } 1}^{\text {rel }}\right)} \sum_{m=0}^{\infty}\left(\frac{\lambda_{\text {rec }} \lambda_{\text {rel }} z}{\left(\lambda_{\text {rec }}+\lambda_{\text {off1 }}^{\text {rec }}\right)\left(\lambda_{\text {rel }}+\lambda_{\text {off } 1}^{\text {rel }}\right)}\right)^{m} .
\end{aligned}
$$

On the other hand, $G(z)$ can be expanded as $G(z)=\sum_{m=0}^{\infty} p_{B S}(m) z^{m}$. Therefore, by identifying the coefficients of the same powers of $z$, we can see that burst size BS follows the following geometric distribution (referring to SI Fig. 11B)

$$
p_{B S}(m)=\theta(1-\theta)^{m}, m=0,1,2, \cdots,
$$


where

$$
\theta=\frac{\lambda_{\text {rec }} \lambda_{\text {off } 1}^{\text {rel }}+\left(\lambda_{\text {rel }}+\lambda_{\text {off } 1}^{\text {rel }}\right) \lambda_{\text {off1 }}^{\text {rec }}}{\left(\lambda_{\text {rec }}+\lambda_{\text {off1 }}^{\text {rec }}\right)\left(\lambda_{\text {rel }}+\lambda_{\text {off1 }}^{\text {rel }}\right)}
$$

Furthermore, Eq. (E41) can be rewritten as

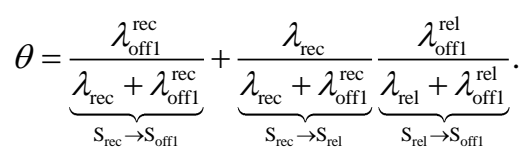

In our model, there are two termination channels whose probability fluxes are expressed perfectly in Eq. (E42). The first term on the right-hand side shows the probability from the state $\mathrm{S}_{\text {rec }}$ directly back to the burst termination state $\mathrm{S}_{\text {offl }}$ so that the burst ends. And the second term reflects that the probability flux is first from the state $S_{\text {rec }}$ to state $S_{\text {rel }}$ and then burst terminates at state $\mathrm{S}_{\mathrm{rel}}$. Therefore, $\theta$ is the success probability of burst termination. Besides, 1- $\theta$ can be expressed as

$$
1-\theta=\underbrace{\frac{\lambda_{\mathrm{rec}}}{\lambda_{\mathrm{rec}}+\lambda_{\text {rec }}^{\text {rec }}}}_{\mathrm{S}_{\mathrm{rec}} \rightarrow \mathrm{S}_{\mathrm{rel}}} \underbrace{\frac{\lambda_{\mathrm{rel}}}{\lambda_{\mathrm{rel}}+\lambda_{\mathrm{off} 1}^{\mathrm{rel}}}}_{\mathrm{S}_{\mathrm{rel}} \rightarrow \mathrm{S}_{\mathrm{rec}}}
$$

which shows the probability flux from state $S_{\text {rec }}$ to state $S_{\text {rel }}$ first and then back to the state $\mathrm{S}_{\text {rel }}$, implying a new mRNA is generated. Thus, $1-\theta$ is the failure probability of burst termination.

In addition, the above geometric distribution of burst size corresponds to the fact that the initial state of each newly generated mRNA is $\mathrm{S}_{\text {rec }}$ and the number of mRNAs accumulates before the burst termination.

And the MBS is given by

$$
\mathrm{MBS}=E[B S]=\frac{\lambda_{\text {rec }} \lambda_{\text {rel }}}{\lambda_{\text {rec }} \lambda_{\text {off } 1}^{\text {rel }}+\left(\lambda_{\text {rel }}+\lambda_{\text {off } 1}^{\text {rel }}\right) \lambda_{\text {off1 }}^{\text {rec }}}
$$

\section{Dwell time, cycle time and burst frequency}

Next, we derive analytical expressions for dwell time and cycle time. First, we calculate the dwell time in each state. Note that the cycle time is equal to the sum of dwell time in all the states.

Following the above analysis process, we first neglect the dwell time in state $\mathrm{S}_{\mathrm{OFF}}$ and then compute the dwell time in state $\mathrm{S}_{\mathrm{ON}}$. Subsequently, the dwell time in the state $\mathrm{S}_{\mathrm{OFF}}$ can 
be calculated separately (SI Fig. 7).

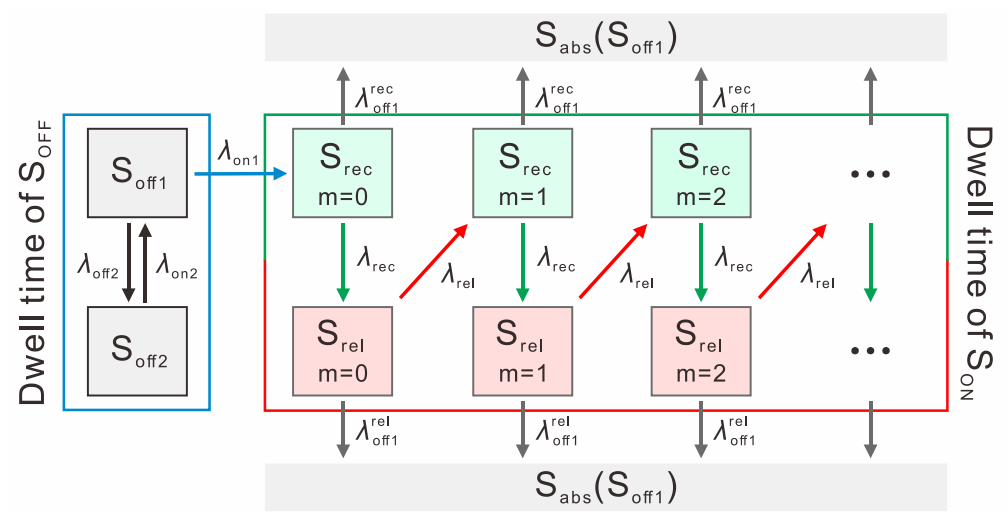

SI Figure 7. Schematic for the transition between discrete states. The notations have the same meaning as in SI Fig. 6.

Based on the discussion of burst size in the previous subsection, the marginal survival probability for time $T$ in Eq. (E23) is

$$
H_{T \mid S}(t \mid s)=\operatorname{Pr}\left(T_{\text {exit }}>t \mid S=s\right),
$$

where $T_{\text {exit }}$ is the exit time from state $s$. For simplicity, we denote $H_{T \mid S}(t \mid s)$ as $H_{s}(t)$.

By using Eq. (E26) and (E45), and by setting $z=1$, we have

$$
G_{s}(1, t)=\sum_{m=0}^{\infty} H_{m, s}(t)=H_{s}(t) .
$$

Thus, the survival probability functions at $S_{\text {rec }}$ and $S_{\text {rel }}$ states equal

$$
\begin{aligned}
& H_{\mathrm{rec}}(t)=G_{\mathrm{rec}}(1, t)=\frac{\left(\lambda_{\mathrm{rel}}+\lambda_{\mathrm{off} 1}^{\mathrm{rel}}+x_{+}\right) e^{x_{+} t}-\left(\lambda_{\mathrm{rel}}+\lambda_{\mathrm{off} 1}^{\mathrm{rel}}+x_{-}\right) e^{x_{-} t}}{x_{+}-x_{-}}, \\
& H_{\mathrm{rel}}(t)=G_{\mathrm{rel}}(1, t)=\frac{\lambda_{\mathrm{rec}}\left(e^{x_{+} t}-e^{x_{-} t}\right)}{x_{+}-x_{-}}
\end{aligned}
$$

where

$$
x_{ \pm}=x_{ \pm}(1)=\frac{1}{2}\left[-\left(\lambda_{\mathrm{rec}}+\lambda_{\mathrm{rel}}+\lambda_{\mathrm{off1}}^{\mathrm{rec}}+\lambda_{\text {off } 1}^{\mathrm{rel}}\right) \pm \sqrt{\left(\left(\lambda_{\mathrm{rec}}+\lambda_{\mathrm{off} 1}^{\mathrm{rec}}\right)-\left(\lambda_{\mathrm{rel}}+\lambda_{\mathrm{off1}}^{\mathrm{rel}}\right)\right)^{2}+4 \lambda_{\mathrm{rec}} \lambda_{\mathrm{rel}}}\right] .
$$

According to the relationship between PDF and survival probability and using Eq. (E47), the dwell time PDFs $p_{\text {rec }}(t)$ and $p_{\text {rel }}(t)$ are

$$
\begin{aligned}
& p_{\text {rec }}(t)=-\frac{\mathrm{d} H_{\text {rec }}(t)}{\mathrm{d} t}=-\frac{\left(\lambda_{\mathrm{rel}}+\lambda_{\text {offl }}^{\mathrm{rel}}+x_{+}\right) x_{+} e^{x_{+} t}-\left(\lambda_{\mathrm{rel}}+\lambda_{\text {off }}^{\mathrm{rel}}+x_{-}\right) x_{-} e^{x_{-} t}}{x_{+}-x_{-}}, \\
& p_{\mathrm{rel}}(t)=-\frac{\mathrm{d} H_{\mathrm{rel}}(t)}{\mathrm{d} t}=-\frac{\lambda_{\mathrm{rec}} x_{+} e^{x_{+} t}-\lambda_{\mathrm{rec}} x_{-} e^{x_{-} t}}{x_{+}-x_{-}} .
\end{aligned}
$$


Since Eq. (E47) is the solution of Eq. (E25), the PDF of the total ON state dwell time, $p_{\mathrm{ON}}(t)$ (note: the complete expression is $p_{D T \mid S}\left(t \mid \mathrm{S}_{\mathrm{ON}}\right)$ ) is given by (referring to SI Fig. 11E)

$$
p_{\mathrm{ON}}(t)=p_{\text {rec }}(t)+p_{\text {rel }}(t)=-\left(A_{+} e^{x_{+} t}-A_{-} e^{x_{-} t}\right)
$$

where

$$
A_{+}=\frac{\left(\lambda_{\mathrm{rec}}+\lambda_{\mathrm{rel}}+\lambda_{\mathrm{off} 1}^{\mathrm{rel}}+x_{+}\right) x_{+}}{x_{+}-x_{-}}, A_{-}=\frac{\left(\lambda_{\mathrm{rec}}+\lambda_{\mathrm{rel}}+\lambda_{\mathrm{offl} 1}^{\mathrm{rel}}+x_{-}\right) x_{-}}{x_{+}-x_{-}} .
$$

The mean ON dwell time $E\left[D T_{\mathrm{ON}}\right]$ (note: the complete expression is $E\left[D T \mid S=\mathrm{S}_{\mathrm{ON}}\right]$ ) can be obtained and the result is

$$
E\left[D T_{\mathrm{ON}}\right]=\int_{0}^{+\infty} t p_{\mathrm{ON}}(t) \mathrm{d} t=\underbrace{\frac{\lambda_{\mathrm{rel}}+\lambda_{\mathrm{off} 1}^{\mathrm{rel}}}{\lambda_{\mathrm{rec}} \lambda_{\mathrm{off1}}^{\mathrm{rel}}+\left(\lambda_{\mathrm{rel}}+\lambda_{\mathrm{off} 1}^{\mathrm{rel}}\right) \lambda_{\mathrm{offl}}^{\mathrm{rec}}}}_{E\left[D T_{\mathrm{rec}}\right]}+\underbrace{\frac{\lambda_{\mathrm{rec}}}{\lambda_{\mathrm{rec}} \lambda_{\mathrm{offl}}^{\mathrm{rel}}+\left(\lambda_{\mathrm{rel}}+\lambda_{\mathrm{offl}}^{\mathrm{rel}}\right) \lambda_{\mathrm{off}}^{\mathrm{rec}}}}_{E\left[D T_{\mathrm{rel}}\right]} .
$$

Next, we compute the PDF of dwell time in $\mathrm{S}_{\mathrm{OFF}}$ state. Owing to the introduction of $\mathrm{S}_{\mathrm{abs}}$, the $\mathrm{S}_{\mathrm{OFF}}$ is a state of the flux that the probability only flows out but does not flow in. By using the same method (the derivation process is omitted here), the dwell time PDF $p_{\text {off } 2}(t)$ and $p_{\text {offl }}(t)$ are found to be

$$
\begin{aligned}
& p_{\text {off } 2}(t)=-\frac{\lambda_{\text {ofr } 2} y_{+} e^{y_{+} t}-\lambda_{\text {off } 2} y_{-} e^{y_{-} t}}{y_{+}-y_{-}}, \\
& p_{\text {off } 1}(t)=-\frac{\left(\lambda_{\text {on } 2}+\lambda_{\text {on1 } 1}+y_{+}\right) y_{+} e^{y_{+} t}-\left(\lambda_{\text {on } 2}+\lambda_{\text {on } 1}+y_{-}\right) y_{-} e^{y_{-} t}}{y_{+}-y_{-}},
\end{aligned}
$$

where

$$
y_{ \pm}=\frac{1}{2}\left[-\left(\lambda_{\mathrm{on} 2}+\lambda_{\mathrm{on} 1}+\lambda_{\mathrm{off} 2}\right) \pm \sqrt{\left(\lambda_{\mathrm{ofr} 2}+\lambda_{\mathrm{on} 1}-\lambda_{\mathrm{on} 2}\right)^{2}+4 \lambda_{\mathrm{on} 2} \lambda_{\mathrm{ofr} 2}}\right] .
$$

The total OFF state dwell time PDF $p_{\mathrm{OFF}}(t)$ (note: the complete expression is $p_{D T \mid S}\left(t \mid \mathrm{S}_{\mathrm{OFF}}\right)$ ) is given by (referring to SI Fig. 11D)

$$
p_{\mathrm{OFF}}(t)=-\left(B_{+} e^{y_{+} t}-B_{-} e^{y_{-} t}\right),
$$

where

$$
B_{+}=\frac{\left(\lambda_{\mathrm{off} 2}+\lambda_{\mathrm{on} 2}+y_{+}\right) y_{+}}{y_{+}-y_{-}}, B_{-}=\frac{\left(\lambda_{\mathrm{off} 2}+\lambda_{\mathrm{on} 2}+y_{-}\right) y_{-}}{y_{+}-y_{-}} .
$$

The mean OFF state dwell time $E\left[D T_{\mathrm{OFF}}\right]$ (note: the complete expression is $E\left[D T \mid S=\mathrm{S}_{\mathrm{OFF}}\right]$ ) 
is

$$
E\left[D T_{\mathrm{OFF}}\right]=\frac{1}{\lambda_{\mathrm{on1}}}+\underbrace{\frac{\lambda_{\mathrm{off} 2}}{\lambda_{\mathrm{on} 2} \lambda_{\mathrm{on1}}}}_{E\left[D T_{\text {off }}\right]} .
$$

Note that the PDF of cycle time $p_{C T}(t)$ is the convolution of $p_{\mathrm{OFF}}(t)$ in Eq. (E55) and $p_{\mathrm{ON}}(t)$ in Eq. (E50). As such, the $p_{C T}(t)$ equals (referring to SI Fig. 11C)

$$
\begin{aligned}
p_{C T}(t) & =\int_{0}^{t} p_{\mathrm{OFF}}(t-w) p_{\mathrm{ON}}(w) \mathrm{d} w \\
& =\frac{A_{+} B_{+}\left(e^{x_{+} t}-e^{y_{+} t}\right)}{x_{+}-y_{+}}+\frac{A_{-} B_{-}\left(e^{x_{-}}-e^{y_{-} t}\right)}{x_{-}-y_{-}}-\frac{A_{-} B_{+}\left(e^{x_{-} t}-e^{y_{+} t}\right)}{x_{-}-y_{+}}-\frac{A_{+} B_{-}\left(e^{x_{+} t}-e^{y_{-} t}\right)}{x_{+}-y_{-}},
\end{aligned}
$$

where $x_{ \pm}, y_{ \pm}, A_{ \pm}$and $B_{ \pm}$are shown in Eq. (E48), (E51), (E54) and (E56). By complex calculation, the MCT is given by

$$
E[C T]=\underbrace{\frac{\lambda_{\text {on2 }}+\lambda_{\text {off2 }}}{\lambda_{\text {on }} \lambda_{\text {on }}}}_{E\left[D T_{\text {orf }}\right]}+\underbrace{\frac{\lambda_{\text {rec }}+\lambda_{\text {rel }}+\lambda_{\text {off1 }}^{\text {rel }}}{\lambda_{\text {rec }} \lambda_{\text {off } 1}+\left(\lambda_{\text {rel }}+\lambda_{\text {off } 1}^{\text {rel }}\right) \lambda_{\text {off }}^{\text {rec }}}}_{E\left[D T_{\text {oN }}\right]} .
$$

Finally, the BF is given by

$$
\mathrm{BF}=\frac{1}{E[C T]}=\frac{\lambda_{\mathrm{on} 2} \lambda_{\mathrm{on} 1}\left[\lambda_{\mathrm{rec}} \lambda_{\mathrm{off} 1}^{\mathrm{rel}}+\left(\lambda_{\mathrm{rel}}+\lambda_{\mathrm{off} 1}^{\mathrm{rel}}\right) \lambda_{\mathrm{off} 1}^{\mathrm{rec}}\right]}{\left(\lambda_{\mathrm{off} 2}+\lambda_{\mathrm{on} 2}\right)\left[\lambda_{\mathrm{rec}} \lambda_{\mathrm{offl}}^{\mathrm{rel}}+\left(\lambda_{\mathrm{rel}}+\lambda_{\mathrm{off} 1}^{\mathrm{rel}}\right) \lambda_{\mathrm{off} 1}^{\mathrm{rec}}\right]+\lambda_{\mathrm{on} 2} \lambda_{\mathrm{on} 1}\left(\lambda_{\mathrm{rec}}+\lambda_{\mathrm{rel}}+\lambda_{\mathrm{off} 1}^{\mathrm{rel} 1}\right)} .
$$

Note that the BF in Eq. (E60) is measured in $\sec ^{-1}$, meaning how many transcriptional bursts occur in one second.

\section{Traveling ratio}

Traveling ratio is defined as the Pol II density ratio between gene body and promoter proximity.

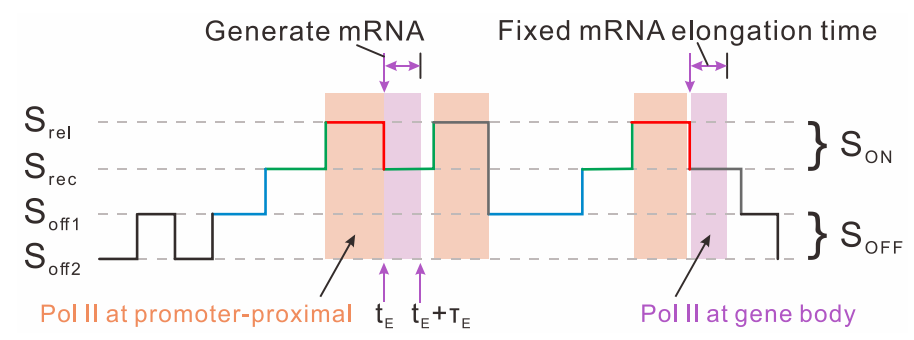

SI Figure 8. Schematic for describing the location and time of Pol II observable in once simulation. Orange block is the time interval of the Pol II at promoter proximity, and purple block is the time interval of the Pol II in gene body.

First, we calculate the Pol II density in gene body, which is the proportion of elongation time in the whole transcriptional burst or the probability that samples fall in the transcriptional 
elongation interval (SI Fig. 8, purple block). We can observe the generation of an mRNA at the time $t_{\mathrm{E}}$ when the state $\mathrm{S}_{\mathrm{rel}}$ switches to state $\mathrm{S}_{\mathrm{rec}}$ and the Pol II elongation at a time interval from $t_{\mathrm{E}}$ to $t_{\mathrm{E}}+\tau_{\mathrm{E}}$ (where $\tau_{\mathrm{E}}$ is a fixed time lag for mRNA elongation). Obviously, the total elongation time equals the elongation time $\left(\tau_{\mathrm{E}}\right)$ of one mRNA times the MBS. Thus, the total elongation time of Pol II can be theoretically expressed as $E[B S] \cdot \tau_{\mathrm{E}}$. The fraction of Pol II in the gene body is the proportion of total elongation time in total cycle time. Based on Eq. (E44) and (E59), and this can be expressed as

$$
P_{\mathrm{GB}}=\frac{E[B S]}{E[C T]} \tau_{\mathrm{E}}=\frac{\lambda_{\mathrm{rec}} \lambda_{\mathrm{rel}} \tau_{\mathrm{E}}}{\lambda_{\mathrm{rec}} \lambda_{\mathrm{off} 1}^{\mathrm{rel}}+\left(\lambda_{\mathrm{rel}}+\lambda_{\mathrm{off1}}\right) \lambda_{\mathrm{offl}}^{\mathrm{rec}}} \frac{1}{E[C T]} .
$$

Next, we consider the Pol II density at promoter proximity, which can be viewed as the proportion of dwell-time of state $S_{\text {rel }}$ in the whole transcriptional burst (SI Fig. $\underline{8}$, orange block). Hence, based on Eq. (E52) and (E59), the fraction of Pol II at promoter proximity can be expressed as

$$
P_{\mathrm{PP}}=\frac{E\left[D T_{\mathrm{rel}}\right]}{E[C T]}=\frac{\lambda_{\mathrm{rec}}}{\lambda_{\mathrm{rec}} \lambda_{\mathrm{offl}}^{\mathrm{rel}}+\left(\lambda_{\mathrm{rel}}+\lambda_{\mathrm{off} 1}^{\mathrm{rel}}\right) \lambda_{\mathrm{offl}}^{\mathrm{rec}}} \frac{1}{E[C T]}
$$

The fraction in Eq. (E62) indicates the probability that the samples are in the state $\mathrm{S}_{\mathrm{rel}}$. This is the stationary probability in the state $\mathrm{S}_{\text {rel }}$. Let $P_{s}(s \in\{$ off 2 , off1, rec, rel $\})$ be the stationary probability of different states. Then, $P_{s}$ satisfies the following equations

$$
\begin{aligned}
& -\lambda_{\mathrm{on} 2} P_{\mathrm{off2}}+\lambda_{\mathrm{off} 2} P_{\mathrm{offl}}=0, \\
& \lambda_{\mathrm{on} 1} P_{\mathrm{offl}}-\left(\lambda_{\mathrm{rec}}+\lambda_{\mathrm{offl}}^{\mathrm{rec}}\right) P_{\mathrm{rec}}+\lambda_{\mathrm{rel}} P_{\mathrm{rel}}=0, \\
& \lambda_{\mathrm{rec}} P_{\mathrm{rec}}-\left(\lambda_{\mathrm{rel}}+\lambda_{\mathrm{offl}}^{\mathrm{rel}}\right) P_{\mathrm{rel}}=0, \\
& P_{\mathrm{off2}}+P_{\mathrm{offl}}+P_{\mathrm{rec}}+P_{\mathrm{rel}}=1,
\end{aligned}
$$

where the last equation holds due to the conservative condition of probability. Solving Eq. (E63), we can obtain $P_{\text {rel }}$ as expressed in Eq. (E62). That is $P_{\mathrm{PP}}=P_{\text {rel }}$.

Finally, the MTR is

$$
E[T R]=\frac{P_{\mathrm{GB}}}{P_{\mathrm{PP}}}=\frac{E[B S]}{E\left[D T_{\mathrm{rel}}\right]} \tau_{\mathrm{E}}=\lambda_{\mathrm{rel}} \tau_{\mathrm{E}}
$$

Note that Eq. (E64) is the ratio of two mean values. However, when calculating MTR, we first need to calculate the traveling ratio at each sampling time, and then take the mean value over 
all sample times. That is $E[T R]=E\left[\frac{B S \cdot \tau_{\mathrm{E}} / C T}{D T_{\text {rel }} / C T}\right]=E\left[\frac{B S}{D T_{\text {rel }}}\right] \cdot \tau_{\mathrm{E}}$. If there are enough samples, we may use approximation $E\left[B S / D T_{\text {rel }}\right] \approx E[B S] / E\left[D T_{\text {rel }}\right]$. Therefore, the mean traveling ratio is given by Eq. (E64). 


\section{F Power laws for transcriptional bursting kinetics}

\section{F1 Binary approximation}

In the previous section, some rates in Eq. (E6) related to E-P spatial distance are described by a piecewise continuous non-binary Hill function in Eq. (C11). The power $h$ of Hill function brings difficulties to the theoretical calculations of MBS, MDT, MCT, and MTR. For this reason, we consider using a simple function to approximate the Hill function. At the simplest level, we may use a deterministic binary rate for transcriptional burst for approximation (SI Fig. 9). That is,

$$
\lambda_{c}\left(d_{\mathrm{S}}\right)= \begin{cases}\lambda_{c, \text { max }}, & d_{\mathrm{S}} \leq \varepsilon_{1 / 2} \\ \lambda_{c, \text { min }}, & d_{\mathrm{S}}>\varepsilon_{1 / 2}\end{cases}
$$

Note that we choose $\varepsilon_{1 / 2}$ instead of $\varepsilon_{\mathrm{T}}$ in Eq. (C11) as the threshold.

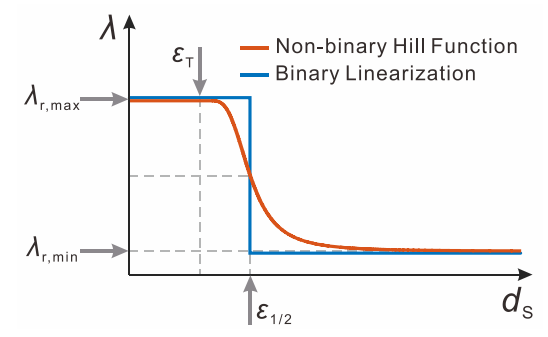

SI Figure 9. Schematic description of the non-binary Hill function and binary linearization.

For binary linearization, only the maximum rate $\lambda_{c, \max }$ and the minimum rate $\lambda_{c, \min }$ are needed. For requirement, we compute $\int p_{D S}\left(d_{\mathrm{S}}\right) \mathrm{d} d_{\mathrm{S}}$ (referring to SI Fig. 10C), which is the CDF of $p_{D S}\left(d_{\mathrm{S}}\right)$ that can be expressed as

$$
F\left(d_{\mathrm{S}}\right)=\operatorname{erf}\left(\frac{d_{\mathrm{S}}}{\sqrt{2} \Theta}\right)-\sqrt{\frac{2}{\pi}} \Theta^{-1} d_{\mathrm{S}} \exp \left(-\frac{d_{\mathrm{S}}^{2}}{2 \Theta^{2}}\right)
$$

where $\operatorname{erf}(\cdot)$ is the error function defined as $\operatorname{erf}(z)=\frac{2}{\sqrt{\pi}} \int_{0}^{z} e^{-t^{2}} \mathrm{~d} t$.

In the case that fluctuations in $d_{\mathrm{S}}$ are much faster compared with the rate of transcription, $\left\langle\lambda_{c}\right\rangle$ may be approximated as $\left\langle\lambda_{c}\right\rangle_{a}$

$$
\left\langle\lambda_{c}\right\rangle_{a}=\lambda_{c, \max } F\left(\varepsilon_{1 / 2}\right)+\lambda_{c, \min }\left(1-F\left(\varepsilon_{1 / 2}\right)\right) .
$$


Then, $\mathrm{MBS}^{\text {Fast }}$ and $\mathrm{MCT}^{\text {Fast }}$ can be approximated as $\mathrm{MBS}_{a}^{\text {Fast }}$ and $\mathrm{MCT}_{a}^{\text {Fast }}$, and

$$
\begin{aligned}
& \mathrm{MBS}_{a}^{\mathrm{Fast}}=\mathrm{BS}_{\text {mid }}=\frac{\left\langle\lambda_{\text {rec }}\right\rangle_{a}\left\langle\lambda_{\text {rel }}\right\rangle_{a}}{\left\langle\lambda_{\mathrm{rec}}\right\rangle_{a} \lambda_{\mathrm{off1} 1}^{\text {rel }}+\left(\left\langle\lambda_{\mathrm{rel}}\right\rangle_{a}+\lambda_{\text {off }}^{\text {rel }}\right) \lambda_{\text {offl }}^{\text {rec }}},
\end{aligned}
$$

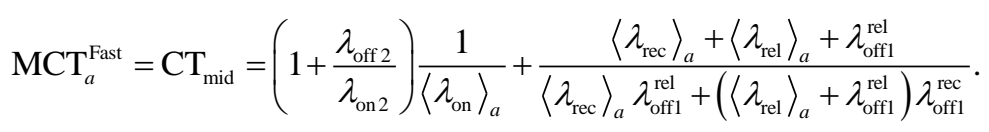

On contrary, if fluctuations in $d_{\mathrm{S}}$ are much slower, the $\mathrm{MBS}^{\text {Slow }}$ and $\mathrm{MCT}^{\text {Slow }}$ can be approximated as $\mathrm{MBS}_{a}^{\text {Slow }}$ and $\mathrm{MCT}_{a}^{\text {Slow }}$, and

$$
\begin{aligned}
& \operatorname{MBS}_{a}^{\text {Slow }}=\mathrm{BS}_{\text {max }} F\left(\varepsilon_{1 / 2}\right)+\mathrm{BS}_{\text {min }}\left(1-F\left(\varepsilon_{1 / 2}\right)\right), \\
& \operatorname{MCT}_{a}^{\text {Slow }}=\mathrm{CT}_{\text {max }} F\left(\varepsilon_{1 / 2}\right)+\mathrm{CT}_{\text {min }}\left(1-F\left(\varepsilon_{1 / 2}\right)\right),
\end{aligned}
$$

where

$$
\begin{aligned}
& \mathrm{BS}_{\text {max }}=\frac{\lambda_{\text {rec,max }} \lambda_{\text {rel,max }}}{\lambda_{\text {rec, } \text { max }} \lambda_{\text {offl }}^{\text {rel }}+\left(\lambda_{\text {rel, max }}+\lambda_{\text {off }}^{\text {rel }}\right) \lambda_{\text {off }}^{\text {rec }}}, \\
& \mathrm{BS}_{\text {min }}=\frac{\lambda_{\text {rec,min }} \lambda_{\text {rel,min }}}{\lambda_{\text {rec, } \min } \lambda_{\text {offl }}^{\text {rl }}+\left(\lambda_{\text {rel, min }}+\lambda_{\text {off } 1}^{\text {rel }}\right) \lambda_{\text {offl }}^{\text {rec }}}, \\
& \mathrm{CT}_{\text {max }}=\left(1+\frac{\lambda_{\text {off } 2}}{\lambda_{\text {on2 } 2}}\right) \frac{1}{\lambda_{\text {on, } \max }}+\frac{\lambda_{\text {rec, max }}+\lambda_{\text {rel, max }}+\lambda_{\text {off1 }}^{\text {rel }}}{\lambda_{\text {rec, } \max } \lambda_{\text {offl }}^{\text {rel }}+\left(\lambda_{\text {rel, max }}+\lambda_{\text {off } 1}^{\text {rel }}\right) \lambda_{\text {off1 }}^{\text {rec }}},
\end{aligned}
$$

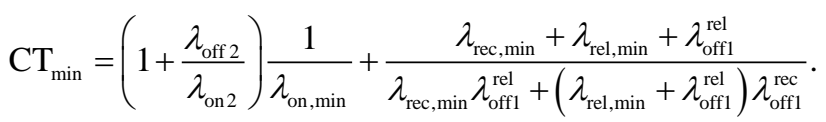

Therefore, based on Eq. (E7) and Eq. (F3) - (F6), the approximations of $\mathrm{MBS}_{a}$ and $\mathrm{MCT}_{a}$ can be expressed as (referring to SI Figs. 14D-E and I-J)

$$
\begin{aligned}
\operatorname{MBS}_{a} & =\frac{1}{1+\omega} \mathrm{MBS}_{a}^{\mathrm{Fast}}+\frac{\omega}{1+\omega} \mathrm{MBS}_{a}^{\text {Slow }}, \\
\mathrm{MCT}_{a} & =\frac{1}{1+\omega} \mathrm{MCT}_{a}^{\mathrm{Fast}}+\frac{\omega}{1+\omega} \mathrm{MCT}_{a}^{\text {Slow }} .
\end{aligned}
$$

\section{F2 Power laws for transcriptional bursting kinetics}

E-P communication strength $k_{\mathrm{EP}}$ and E-P genomic distance $d_{\mathrm{G}}$ are two key parameters in our model. Note that $F$ in Eq. (F2) can be regarded as a function of three independent variables $k_{\mathrm{EP}}, d_{\mathrm{G}}$ and $d_{S}$, and may be written as $F\left(k_{\mathrm{EP}}, d_{\mathrm{G}}, d_{\mathrm{S}}\right)$. Therefore, MBS and MCT in Eq. (E7) can be also treated as multivariate functions, denoted by $\operatorname{MBS}\left(\lambda_{c, \text { max }}, \lambda_{c, \text { min }}, k_{\mathrm{EP}}, d_{\mathrm{G}}, d_{\mathrm{S}}\right)$ and $\operatorname{MCT}\left(\lambda_{c, \text { max }}, \lambda_{c, \text { min }}, k_{\mathrm{EP}}, d_{\mathrm{G}}, d_{\mathrm{S}}\right)$, respectively. In order to show 
the effects of increasing $k_{\mathrm{EP}}$ or $d_{\mathrm{G}}$ on $\mathrm{MBS}$ and $\mathrm{BF}$, we calculate derivatives: $\frac{\partial \log _{10} \mathrm{MBS}}{\partial \log _{10} k_{\mathrm{EP}}}$ and $\frac{\partial \log _{10} \mathrm{BF}}{\partial \log _{10} k_{\mathrm{EP}}}, \frac{\partial \log _{10} \mathrm{MBS}}{\partial \log _{10} d_{\mathrm{G}}}$ and $\frac{\partial \log _{10} \mathrm{BF}}{\partial \log _{10} d_{\mathrm{G}}}$.

Using Eq. (F2), we know that $\frac{\partial F\left(k_{\mathrm{EP}}, d_{\mathrm{G}}, d_{\mathrm{S}}\right)}{\partial k_{\mathrm{EP}}}$ and $\frac{\partial F\left(k_{\mathrm{EP}}, d_{\mathrm{G}}, d_{\mathrm{S}}\right)}{\partial d_{\mathrm{G}}}$ are

$$
\frac{\partial F\left(k_{\mathrm{EP}}, d_{\mathrm{G}}, d_{\mathrm{S}}\right)}{\partial k_{\mathrm{EP}}}=\frac{1}{\sqrt{2 \pi}}\left(\frac{d_{\mathrm{S}}}{\sqrt{D \gamma}}\right)^{3} \sqrt{\frac{k_{\mathrm{NN}}}{d_{\mathrm{G}}}+k_{\mathrm{EP}}} \exp \left(-\frac{1}{2}\left(\frac{d_{\mathrm{S}}}{\sqrt{D \gamma}}\right)^{2}\left(\frac{k_{\mathrm{NN}}}{d_{\mathrm{G}}}+k_{\mathrm{EP}}\right)\right),
$$

and

$$
\frac{\partial F\left(k_{\mathrm{EP}}, d_{\mathrm{G}}, d_{\mathrm{S}}\right)}{\partial d_{\mathrm{G}}}=-\frac{k_{\mathrm{NN}}}{d_{\mathrm{G}}^{2}} \frac{\partial F\left(k_{\mathrm{EP}}, d_{\mathrm{G}}, d_{\mathrm{S}}\right)}{\partial k_{\mathrm{EP}}}
$$

In the following, for the sake of simplicity, we rewrite $\left.F\left(k_{\mathrm{EP}}, d_{\mathrm{G}}, d_{\mathrm{S}}\right)\right|_{d_{\mathrm{s}}=\varepsilon_{1 / 2}}$ as $F\left(k_{\mathrm{EP}}\right)$ $\left(F\left(d_{\mathrm{G}}\right)\right)$ when analyzing the effect of $k_{\mathrm{EP}}\left(d_{\mathrm{G}}\right)$. Note that the following theoretical derivation is for the case of $d_{\mathrm{G}}=50$ (when we consider $k_{\mathrm{EP}}$ ) and $k_{\mathrm{EP}}=0.1$ (when we consider $d_{\mathrm{G}}$ ). Of course, the corresponding theoretical derivation method is still applicable to cases of other parameter values.

\section{Power law in terms of E-P communication strength}

The effect of changing $k_{\mathrm{EP}}$ on MBS can be approximated as

$$
\frac{\partial \log _{10} \mathrm{MBS}}{\partial \log _{10} k_{\mathrm{EP}}} \approx \frac{k_{\mathrm{EP}}}{\mathrm{MBS}} \frac{\partial \mathrm{MBS}_{a}}{\partial k_{\mathrm{EP}}},
$$

where

$$
\frac{\partial \mathrm{MBS}_{a}}{\partial k_{\mathrm{EP}}}=\left[\left.\frac{1}{1+\omega} \sum_{c} \frac{\partial \mathrm{MBS}_{a}^{\mathrm{Fast}}\left(\lambda_{c}\right)}{\partial \lambda_{c}}\right|_{\lambda_{c}=\left\langle\lambda_{c}\right\rangle_{a}}\left(\lambda_{c, \text { max }}-\lambda_{c, \text { min }}\right)+\frac{\omega}{1+\omega}\left(\mathrm{BS}_{\text {max }}-\mathrm{BS}_{\text {min }}\right)\right] \frac{\partial F\left(k_{\mathrm{EP}}\right)}{\partial k_{\mathrm{EP}}},
$$

and $c \in\{$ on1,rec,rel $\}$.

Similarly, the effect of changing $k_{\mathrm{EP}}$ on $\mathrm{BF}$ is

$$
\frac{\partial \log _{10} \mathrm{BF}}{\partial \log _{10} k_{\mathrm{EP}}}=-\frac{\partial \log _{10} \mathrm{MCT}}{\partial \log _{10} k_{\mathrm{EP}}} \approx-\frac{k_{\mathrm{EP}}}{\mathrm{MCT}} \frac{\partial \mathrm{MCT}_{a}}{\partial k_{\mathrm{EP}}},
$$

where

$$
\frac{\partial \mathrm{MCT}_{a}}{\partial k_{\mathrm{EP}}}=\left[\left.\frac{1}{1+\omega} \sum_{c} \frac{\partial \mathrm{MCT}_{a}^{\mathrm{Fast}}\left(\lambda_{c}\right)}{\partial \lambda_{c}}\right|_{\lambda_{c}=\left\langle\lambda_{c}\right\rangle_{a}}\left(\lambda_{c, \text { max }}-\lambda_{c, \text { min }}\right)+\frac{\omega}{1+\omega}\left(\mathrm{CT}_{\text {max }}-\mathrm{CT}_{\text {min }}\right)\right] \frac{\partial F\left(k_{\mathrm{EP}}\right)}{\partial k_{\mathrm{EP}}},
$$

and $c \in\{$ on1,rec,rel $\}$.

Note that both $\frac{\partial \log _{10} \mathrm{MBS}}{\partial \log _{10} k_{\mathrm{EP}}}$ and $\frac{\partial \log _{10} \mathrm{BF}}{\partial \log _{10} k_{\mathrm{EP}}}$ are positive when the parameters are not 
extreme. They increase for small $k_{\mathrm{EP}}$ and then quickly decrease to 0 with increasing $k_{\mathrm{EP}}$. Meanwhile, the value of the derivatives changes little (the maximum values are not more than 0.8, SI Figs. 14A-C), implying that linear approximation is appropriate for $\log _{10} \mathrm{MBS}\left(\log _{10} k_{\mathrm{EP}}\right)$ and $\log _{10} \mathrm{BF}\left(\log _{10} k_{\mathrm{EP}}\right)$ within an appropriate range of $k_{\mathrm{EP}}$.

Assume that we can obtain distribution $F\left(k_{\mathrm{EP}_{1}}\right) \approx 1$ when $k_{\mathrm{EP}_{1}}$ is greater than a pre-given value, and $\lim _{k_{\mathrm{EP}_{2}} \rightarrow 0} F\left(k_{\mathrm{EP}_{2}}\right) \approx 0.0224$ where $d_{\mathrm{G}}=50$. We define

$$
S_{\mathrm{BS}}=\frac{\log _{10} \operatorname{MBS}\left(k_{\mathrm{EP}_{1}}\right)-\log _{10} \mathrm{MBS}\left(k_{\mathrm{EP}_{2}}\right)}{\log _{10} k_{\mathrm{EP}_{1}}-\log _{10} k_{\mathrm{EP}_{2}}},
$$

which represents the slope of the line of $\log _{10}$ MBS vs $\log _{10} k_{\mathrm{EP}}$. Then, we obtain the following an approximate linear relation:

$$
\begin{aligned}
\log _{10} \operatorname{MBS}\left(k_{\mathrm{EP}}\right) & \approx S_{\mathrm{BS}} \cdot\left(\log _{10} k_{\mathrm{EP}}-\log _{10} k_{\mathrm{EP}}\right)+\log _{10}\left(\mathrm{BS}_{\max }\right) \\
& =S_{\mathrm{BS}} \cdot \log _{10} k_{\mathrm{EP}}+\log _{10}\left(\mathrm{BS}_{\text {max }}\right),
\end{aligned}
$$

which implies that mean burst size obeys the following power law:

$$
\mathrm{MBS} \sim\left(k_{\mathrm{EP}}\right)^{S_{\mathrm{BS}}} .
$$

Similarly, if we define

$$
S_{\mathrm{BF}}=-\frac{\log _{10} \mathrm{MCT}\left(k_{\mathrm{EP}_{1}}\right)-\log _{10} \mathrm{MCT}\left(k_{\mathrm{EP}_{2}}\right)}{\log _{10} k_{\mathrm{EP}_{1}}-\log _{10} k_{\mathrm{EP}_{2}}},
$$

then

$$
\begin{aligned}
\log _{10} \mathrm{BF}\left(k_{\mathrm{EP}}\right) & \approx S_{\mathrm{BF}} \cdot\left(\log _{10} k_{\mathrm{EP}}-\log _{10} k_{\mathrm{EP}_{1}}\right)-\log _{10}\left(\mathrm{CT}_{\max }\right) \\
& =S_{\mathrm{BF}} \cdot \log _{10} k_{\mathrm{EP}}-\log _{10}\left(\mathrm{CT}_{\max }\right),
\end{aligned}
$$

which implies that mean burst frequency obeys the following power law:

$$
\mathrm{BF} \sim\left(k_{\mathrm{EP}}\right)^{S_{\mathrm{BF}}} .
$$

\section{Power law in terms of E-P genomic distance}

Similarly, we can also compute $\frac{\partial \log _{10} \text { MBS }}{\partial \log _{10} d_{\mathrm{G}}}$ and $\frac{\partial \log _{10} \mathrm{BF}}{\partial \log _{10} d_{\mathrm{G}}}$, that is,

$$
\frac{\partial \log _{10} \mathrm{MBS}}{\partial \log _{10} d_{\mathrm{G}}} \approx \frac{d_{\mathrm{G}}}{\mathrm{MBS}} \frac{\partial \mathrm{MBS}_{a}}{\partial d_{\mathrm{G}}}, \frac{\partial \log _{10} \mathrm{BF}}{\partial \log _{10} d_{\mathrm{G}}} \approx-\frac{d_{\mathrm{G}}}{\mathrm{MCT}} \frac{\partial \mathrm{MCT}_{a}}{\partial d_{\mathrm{G}}},
$$

where 


$$
\begin{aligned}
\frac{\partial \mathrm{MBS}_{a}}{\partial d_{\mathrm{G}}} & =\left[\left.\frac{1}{1+\omega} \sum_{c} \frac{\partial \mathrm{MBS}_{a}^{\mathrm{Fast}}\left(\lambda_{c}\right)}{\partial \lambda_{c}}\right|_{\lambda_{c}=\left\langle\lambda_{c}\right\rangle_{a}}\left(\lambda_{c, \text { max }}-\lambda_{c, \text { min }}\right)+\frac{\omega}{1+\omega}\left(\mathrm{BS}_{\max }-\mathrm{BS}_{\text {min }}\right)\right] \frac{\partial F\left(d_{\mathrm{G}}\right)}{\partial d_{\mathrm{G}}}, \\
\frac{\partial \mathrm{MCT}_{a}}{\partial d_{\mathrm{G}}} & =\left[\left.\frac{1}{1+\omega} \sum_{c} \frac{\partial \mathrm{MCT}_{a}^{\mathrm{Fast}}\left(\lambda_{c}\right)}{\partial \lambda_{c}}\right|_{\lambda_{c}=\left\langle\lambda_{c}\right\rangle_{a}}\left(\lambda_{c, \text { max }}-\lambda_{c, \text { min }}\right)+\frac{\omega}{1+\omega}\left(\mathrm{CT}_{\max }-\mathrm{CT}_{\text {min }}\right)\right] \frac{\partial F\left(d_{\mathrm{G}}\right)}{\partial d_{\mathrm{G}}},
\end{aligned}
$$

and $c \in\{$ on1,rec,rel $\}$.

Based on Eq. (F9), we know that the monotonicity of $\frac{\partial \log _{10} \mathrm{MBS}}{\partial \log _{10} d_{\mathrm{G}}}$ and $\frac{\partial \log _{10} \mathrm{BF}}{\partial \log _{10} d_{\mathrm{G}}}$ is opposite that of $\frac{\partial \log _{10} \mathrm{MBS}}{\partial \log _{10} k_{\mathrm{EP}}}$ and $\frac{\partial \log _{10} \mathrm{BF}}{\partial \log _{10} k_{\mathrm{EP}}}$, respectively. Moreover, $\frac{\partial \log _{10} \mathrm{MBS}}{\partial \log _{10} d_{\mathrm{G}}}$ and $\frac{\partial \log _{10} \mathrm{BF}}{\partial \log _{10} d_{\mathrm{G}}}$ decrease for small $d_{\mathrm{G}}$ and then quickly increase to 0 with increasing $d_{\mathrm{G}}$. Meanwhile, the derivatives value changes little (the minimum values are not less than -0.6, SI Figs. 14F-H), implying that linear approximation is appropriate for $\log _{10} \mathrm{MBS}\left(\log _{10} d_{\mathrm{G}}\right)$ and $\log _{10} \mathrm{BF}\left(\log _{10} d_{\mathrm{G}}\right)$ within an appropriate range of $d_{\mathrm{G}}$.

Assume that we can obtain distribution $F\left(d_{\mathrm{G}_{1}}=1\right)=0.9883$ where 1 is the minimum of $d_{\mathrm{G}}$ and $k_{\mathrm{EP}}=0.1$. Numerical results indicate that the turning point for the biphasic responses of burst size and burst frequency to $d_{\mathrm{G}}$ is different. We denote by $d_{\mathrm{G}_{2}}^{\mathrm{BS}}$ and $d_{\mathrm{G}_{2}}^{\mathrm{BF}}$ the $d_{\mathrm{G}}$ values corresponding to the turning points of burst size and burst frequency respectively. And we define

$$
S_{\mathrm{BS}}=\frac{\log _{10} \operatorname{MBS}\left(d_{\mathrm{G}_{1}}\right)-\log _{10} \operatorname{MBS}\left(d_{\mathrm{G}_{2}}^{\mathrm{BS}}\right)}{\log _{10} d_{\mathrm{G}_{1}}-\log _{10} d_{\mathrm{G}_{2}}^{\mathrm{BS}}}
$$

which represents the slope of the line of $\log _{10}$ MBS vs $\log _{10} k_{\mathrm{EP}}$. Then, we obtain the following approximate linear relation when $d_{\mathrm{G}} \leq d_{\mathrm{G}_{2}}^{\mathrm{BS}}$

$$
\begin{aligned}
\log _{10} \operatorname{MBS}\left(d_{\mathrm{G}}\right) & \approx S_{\mathrm{BS}} \cdot\left(\log _{10} d_{\mathrm{G}}-\log _{10} d_{\mathrm{G}_{1}}\right)+\log _{10}\left(\mathrm{BS}_{\max }\right) \\
& =S_{\mathrm{BS}} \cdot \log _{10} d_{\mathrm{G}}+\log _{10}\left(\mathrm{BS}_{\max }\right),
\end{aligned}
$$

which implies that mean burst size obeys the following power law:

$$
\mathrm{MBS} \sim\left(d_{\mathrm{G}}\right)^{S_{\mathrm{BS}}} .
$$

For $\log _{10} \mathrm{BF}$, if we define

$$
S_{\mathrm{BF}}=-\frac{\log _{10} \operatorname{MCT}\left(d_{\mathrm{G}_{1}}\right)-\log _{10} \operatorname{MCT}\left(d_{\mathrm{G}_{2}}^{\mathrm{BF}}\right)}{\log _{10} d_{\mathrm{G}_{1}}-\log _{10} d_{\mathrm{G}_{2}}^{\mathrm{BF}}},
$$


then when $d_{\mathrm{G}} \leq d_{\mathrm{G}_{2}}^{\mathrm{BF}}$, we have

$$
\begin{aligned}
\log _{10} \mathrm{BF}\left(d_{\mathrm{G}}\right) & \approx S_{\mathrm{BF}} \cdot\left(\log _{10} d_{\mathrm{G}}-\log _{10} d_{\mathrm{G}_{1}}\right)-\log _{10}\left(\mathrm{CT}_{\max }\right) \\
& =S_{\mathrm{BF}} \cdot \log _{10} d_{\mathrm{G}}-\log _{10}\left(\mathrm{CT}_{\text {max }}\right),
\end{aligned}
$$

which implies that mean burst frequency obeys the following power law:

$$
\mathrm{BF} \sim\left(d_{\mathrm{G}}\right)^{S_{\mathrm{BF}}} .
$$

Numerical simulations including analysis of experimental data have verified these power-law behaviors, referring to SI Fig. 16. 


\section{G E-P communication mainly modulates burst frequency}

In order to theoretically investigate which of burst size and burst frequency is more affected than the other by E-P communication, we consider the ratio

$$
\frac{S_{\mathrm{BS}}}{S_{\mathrm{BF}}}=-\frac{\log _{10} \operatorname{MBS}\left(k_{\mathrm{EP}_{1}}\right)-\log _{10} \operatorname{MBS}\left(k_{\mathrm{EP}_{2}}\right)}{\log _{10} \operatorname{MCT}\left(k_{\mathrm{EP}_{1}}\right)-\log _{10} \operatorname{MCT}\left(k_{\mathrm{EP}_{2}}\right)} .
$$

Note that for enhancer deletion, we have $k_{\mathrm{EP}_{2}}=0$. In this case, the transcriptional burst rates are always the minimums and are independent of E-P spatial distance $d_{\mathrm{S}}$. Then, Eq. (G1) can be written as

$$
\frac{S_{\mathrm{BS}}^{\mathrm{E}}}{S_{\mathrm{BF}}^{\mathrm{E}}}=-\frac{\log _{10} \operatorname{MBS}\left(k_{\mathrm{EP}_{1}}\right)-\log _{10} \operatorname{MBS}(0)}{\log _{10} \operatorname{MCT}\left(k_{\mathrm{EP}_{1}}\right)-\log _{10} \operatorname{MCT}(0)} .
$$

In the main text, we have mapped the high-dimensional parameter space into an experimentally measurable and theoretically computable two-dimensional space. The key is that we take ratios $\rho_{\mathrm{BS}}=\frac{\mathrm{BS}_{\max }}{\mathrm{BS}_{\min }}$ and $\rho_{\mathrm{BF}}=\frac{\mathrm{CT}_{\min }}{\mathrm{CT}_{\max }}$ as the coordinates in two-dimensional space, where the involved $\mathrm{BS}_{\max }, \mathrm{BS}_{\min }, \mathrm{CT}_{\min }, \mathrm{CT}_{\max }$ are defined in Eq. (F6). 


\section{H Calculation of mutual information}

Many molecular regulations can impact chromatin conformation. For example, because the hypervariable genes are estrogen-responsive and estrogen induces changes in E-P contacts (90, 91), this leads to genome-wide chromosome conformation changes in response to external stimulations (92). A more detailed study is that by experimentally investigating the endogenous regulation of the estrogen-responsive TFF1 gene, J. Rodriguez et al observed that topologically associated domains appear denser in contacts overall, as reported recently (93). They also observed increases in off-diagonal contact frequency, characteristic of defined enhancerpromoter interactions. However, many estrogen response elements did not show such obvious enrichments between two specific regions, but increased the contact probability with multiple parts of the chromosome and a concomitant loss of contacts with many other regions.

In principle, we may use Shannon entropy to measure the effect of this information propagation on the resulting transcriptional bursting. However, to put this quantitative entropy change in a biological context, here we calculate the mutual information (MI) between downstream random variables $X \in\{B S, D T \mid S, C T\}$ and upstream E-P spatial distance $D S$, denoted by $\operatorname{MI}(X, D S)$, such as $\operatorname{MI}(C T, D S)$. Specifically, we define the mutual information as (referring to SI Fig. 18)

$$
\operatorname{MI}(X, D S)=\int_{0}^{+\infty} \int_{0}^{+\infty} p_{X, D S}\left(x, d_{\mathrm{S}}\right) \log \left(\frac{p_{X, D S}\left(x, d_{\mathrm{S}}\right)}{p_{X}(x) p_{D S}\left(d_{\mathrm{S}}\right)}\right) \mathrm{d} \mathrm{d} \mathrm{d} d_{\mathrm{S}},
$$

where $p_{X}(x)$ are given in Eq. (E6), $p_{D S}\left(d_{\mathrm{S}}\right)$ are shown in Eq. (E14), and $p_{X, D S}\left(x, d_{\mathrm{S}}\right)$ can be calculated according to $p_{X, D S}\left(x, d_{\mathrm{S}}\right)=\frac{1}{1+\omega} p_{X, D S}^{\text {Fast }}\left(x, d_{\mathrm{S}}\right)+\frac{\omega}{1+\omega} p_{X, D S}^{\text {Slow }}\left(x, d_{\mathrm{S}}\right)$, where $p_{X, D S}^{\text {Fast }}\left(x, d_{S}\right)$ and $p_{X, D S}^{\text {Slow }}\left(x, d_{S}\right)$ are given in Eq. (E2) and Eq. (E4), respectively. When we calculate $\operatorname{MI}(B S, D S)$, it is necessary to replace the integral symbol in Eq. (H1) with the summation symbol since $B S$ is a discrete random variable.

We find the $\operatorname{MI}(X, D S)$ value is in general small. This may be because of the following three reasons. First, our model only considers one E-P pair, but in the real organisms, multiple 
pairs can be formed. And multiple genes or multipair E-P communication generally transduce more information than a single E-P communication (94). Second, multiple output distributions such as burst size distribution and cycle time distribution make it possible to separately measure the amount of the information transduced from the E-P communication signal to outcome distributions. This would lead to smaller $\mathrm{MI}(X, D S)$ than calculating the mutual information between the input distribution and the gene expression distribution that integrates BS's and CT's information. The third possible reason is that the timescale difference between the upstream and the downstream influences the size of mutual information, e.g., if the upstream motion is much faster than the downstream but the information arriving at the downstream is the average, the upstream and the downstream are independent and the mutual information between them is zero. However, the smaller mutual information does not mean that E-P communication is meaningless but may reflect the robustness of E-P communication. 


\section{Biologically reasonable setting of model parameter values}

We set the values of our model parameters mainly based on experimental results on Drosophila.

SI Table 2: List of model parameter values

\begin{tabular}{|c|c|c|c|}
\hline Param & Description & Value & Ref \\
\hline \multicolumn{4}{|c|}{ Chromatin motion } \\
\hline$\Delta t$ & Time step & $0.01 s$ & $(28,29)$ \\
\hline$k_{B}$ & Boltzmann constant & $1.38 \times 10^{-23} J^{-1}$ & --- \\
\hline$T$ & Room temperature & $300 \mathrm{~K}$ & --- \\
\hline$D$ & Monomer diffusion coefficient & $4 \times 10^{-4} \mu m^{2} s^{-1}$ & $(95)$ \\
\hline$\gamma$ & Friction coefficient & $10 p N s \mu m^{-1}$ & $D \gamma=k_{B} T$ \\
\hline$b$ & Standard deviation distance between monomers & $0.1 \mu m$ & (28) \\
\hline$k_{\mathrm{NN}}$ & Spring constant between adjacent monomers & $1 p N \mu m^{-1}$ & $k_{\mathrm{NN}}=3 k_{B} T / b^{2}$ \\
\hline$k_{\mathrm{EP}}$ & E-P interaction coefficient & free & --- \\
\hline \multicolumn{4}{|c|}{ Transcription } \\
\hline$\lambda_{\text {on2 }}$ & Chromatin opening rate & $0.002 s^{-1}$ & $(56)$ \\
\hline$\lambda_{\text {off2 }}$ & Chromatin closure rate & $0.006 s^{-1}$ & $(56)$ \\
\hline$\lambda_{\text {on1 }}$ & Transcriptional initiation rate (from min to max) & $0.002 \sim 0.020 s^{-1}$ & (96) \\
\hline$\lambda_{\mathrm{rec}}$ & Pol II recruitment rate (from min to max) & $0.009 \sim 0.024 s^{-1}$ & $(97)$ \\
\hline$\lambda_{\text {rel }}$ & Pol II pause release rate (from min to max) & $0.008 \sim 0.025 s^{-1}$ & $(97)$ \\
\hline$\lambda_{\text {off }}^{\mathrm{rec}}$ & Transcriptional termination rate (channel 1) & $0.009 s^{-1}$ & $(38,96)$ \\
\hline$\lambda_{\text {off } 1}^{\text {rel }}$ & Transcriptional termination rate (channel 2) & $0.002 s^{-1}$ & $(38,96)$ \\
\hline$h$ & Hill coefficient & 3 & $(98)$ \\
\hline$\varepsilon_{1 / 2}$ & Half-value distance of burst rates & $0.2 \mu m$ & $(37)$ \\
\hline$\varepsilon_{\mathrm{T}}$ & E-P encounter distance (distance threshold) & $0.1 \mu m$ & $b$ \\
\hline$r_{\mathrm{E}}$ & Transcriptional elongation rate & $30 \operatorname{bps}^{-1}$ & $(99-102)$ \\
\hline$L_{\mathrm{E}}$ & Elongation length (gene body length) & $3000 b p$ & $(103)$ \\
\hline$\tau_{\mathrm{E}}$ & Transcriptional elongation time & $100 s$ & $\tau_{E}=L_{E} / r_{E}$ \\
\hline \multicolumn{4}{|c|}{ Other parameters } \\
\hline$l$ & Nucleosome DNA length & $200 b p$ & $(104)$ \\
\hline$L$ & Genome length & $20 k b p$ & $(105)$ \\
\hline
\end{tabular}




\begin{tabular}{|l|l|l|l|}
\hline$N$ & Number of monomers & 100 & $N=L / l$ \\
\hline
\end{tabular}

Drosophila, one of the intensively studied organisms in the biological field, serves as a test system to explore cellular processes including transcriptional bursting. To investigate the power of our model, it is needed to discuss how the model parameters to fit experimental conditions.

\section{Enhancer and promoter lengths}

Enhancers and promoters are regulatory elements in transcriptional activation and bursting, and their lengths are highly variable in different organisms or genes.

The length of an enhancer can vary from about $50 \mathrm{bp}$ to $1.5 \mathrm{kbp}$ (106) and typically, the metazoan enhancer is $\sim 500$ bp (107). For example, in Drosophila embryo, the rhomboid (rho) enhancer is $300 \mathrm{bp}$ in length, the brinker (brk) and vein $(v n)$ enhancers are $500 \mathrm{bp}$ in length (107) and the enhancer of $l a b$ gene contains a $550 \mathrm{bp}$ fragment (108). Each enhancer contains specific motifs and multiple binding sites for TFs, and these motifs assemble within a $\sim 300 \mathrm{bp}$ core domain (107). Therefore, we stipulate that the enhancer length is $300 \mathrm{bp}$ in simulations.

Promoters can be divided into peaked and broad promoters based on their widths. And promoter length is also highly variable. In Drosophila, the median width of broad promoters is $162 \mathrm{bp}$ (109). An experimental program obtained $422 \mathrm{kbp}$ promoter sequences from 2424 genes in Drosophila (110), and then we can calculate that one promoter is about $174 \mathrm{bp}$ in length. Thus, we assume the promoter length is $170 \mathrm{bp}$ in simulations.

The length of DNA around a nucleosome is about $167 \mathrm{bp}$, which is approximate to that of a promoter. In contrast, the length of the linker DNA is more variable, ranging from $10 \mathrm{bp}$ to $90 \mathrm{bp}(104,111)$. Thus, the length of DNA in one nucleosome plus the length of the linker DNA at both sides of the nucleosome is approximately equal to that of the enhancer. Overall, we can use one nucleosome indicates an enhancer or a promoter.

\section{E-P genomic distance and the number of monomers}

Along the linear genome, the distances between the enhancers and their cognate promoters vary greatly. In Drosophila, this distance can be more than $100 \mathrm{kbp}$. The median distance is 10 $\mathrm{kbp}$ and the most possible distance of an E-P pair is $5 \mathrm{kbp}$ (105). The distances from more than $70 \%$ of enhancers to target transcriptional start sites do not exceed $20 \mathrm{kbp}$. Therefore, we set the total genome length $L$ as $20 \mathrm{kbp}$ in simulations. And the E-P genomic distance is set to be $10 \mathrm{kbp}$ by default. When considering the effect of E-P genomic distance on transcriptional 
bursting, we let the E-P genomic distance increase from $1 \mathrm{kbp}$ to $20 \mathrm{kbp}$. In simulations, we assume that one monomer represents a nucleosome ( $200 \mathrm{bp})$. Thus, the number of monomers $N$ is 100 .

\section{Spring coefficient between adjacent monomers and monomer diffusion coefficient}

The Boltzmann constant equals $k_{B}=1.38 \times 10^{-23} \mathrm{~J} / \mathrm{K}$ and we set temperature $T$ as $300 \mathrm{~K}$ (room temperature). Thus, $k_{B} T \approx 4 \times 10^{-3} p N \mu m$. The standard deviation of the distance between adjacent monomers is at the micron level (28). Therefore, we posit $b=0.1 \mu m$. According to the relation $k_{\mathrm{NN}}=d k_{B} T / b^{2}$, we can compute the spring coefficient for successive monomers, i.e., $k_{\mathrm{NN}}=1 p N / \mu m$.

Owing to the Einstein relation $D \gamma=k_{B} T$ and the fact that the E-P spatial distance PDF is affected by the product of $D$ and $\gamma$ (see Eq. (E14)), we may not care about the respective values of $D$ and $\gamma$, but set their product as $k_{B} T \approx 4 \times 10^{-3} p N \mu m$. We set the monomer diffusion coefficient of DNA as $D=4 \times 10^{-4} \mu \mathrm{m}^{2} / \mathrm{s}$ and thus the friction coefficient is $\gamma=10 \mathrm{pNs} / \mu \mathrm{m}$. Of course, for $D$ and $\gamma$, we can set different values, but $D \gamma=k_{B} T$ must be guaranteed. It is worth noting that the size of $\gamma$ affects the timescale of upstream chromatin motion.

\section{E-P communication strength}

In Drosophila embryos (37), the difference between the E-P distances of different experiments can reach up to $1 \mu \mathrm{m}$. The E-P interaction coefficient $k_{\mathrm{EP}}$ may change in the range of $0-1 p N / \mu m$, so we can study the effects of distinct $k_{\mathrm{EP}}$ on bursting kinetics. Note that $k_{\mathrm{EP}}=0$ means that there is no communication between enhancer and promoter. Also, it is unnecessary to consider the case of larger $k_{\mathrm{EP}}(>1 \mathrm{pN} / \mu \mathrm{m})$ because the bursting dynamics do not change with the increase $k_{\mathrm{EP}}$ when $k_{\mathrm{EP}}>1 p N / \mu m$. The $k_{\mathrm{EP}}$ is set to be $k_{\mathrm{EP}}=0.1 p N / \mu m$ by default in the main text.

\section{Hill function}

The Hill coefficient $H$ measures ultra-sensitivity. In biology, the $H$ usually changes from 2 to $5(98,112)$. We fix $h=3$ for the studies in the main text. The E-P encounter distance is $\varepsilon_{\mathrm{T}}=0.1 \mu \mathrm{m}$, which equals to the standard deviation of the distance between adjacent 
monomers $b$ and approximately equals to $0.12 \mu m$ in (113).

E-P communication or E-P proximity is necessary for transcriptional bursting. The E-P distance is about $0.2 \sim 0.4 \mu \mathrm{m}$ in bursting $(25,37,72,82)$. When E-P spatial distance is larger than $0.4 \mu m$, the burst rates tend to the minimum. Considering this, we assume the half-value distance of burst rates is $\varepsilon_{1 / 2}=0.2 \mu \mathrm{m}$, and thus the rates drop to the minimum around $0.4 \mu \mathrm{m}$.

\section{Gene states switching rates}

In Drosophila, the burst duration is about 10-20 minutes $(14,96,114,115)$. The sm-FISH has shown that in Drosophila gap genes, the mRNA is transcribed with the average residence time $\approx 3 \mathrm{~min}$ and the gene is not expressed with a relatively longer lifetime.

In Section E, we analytically showed that the OFF-state dwell time is $\left(1+\frac{\lambda_{\text {off } 2}}{\lambda_{\text {on } 2}}\right) \frac{1}{\lambda_{\text {on } 1}}$. For simplicity, we assume that the relationship between $\lambda_{\text {off2 }}$ and $\lambda_{\text {on2 }}$ is multiple (56), e.g., $\lambda_{\text {off } 2}=3 \lambda_{\text {on } 2}$. Thus, the OFF-state dwell time is $4 / \lambda_{\text {on1 }}$. We can assume that the fluctuating range of the transcriptional initial rate is from $\lambda_{\text {on1, } \min }=0.002 s^{-1}$ to $\lambda_{\text {onl, } \max }=0.020 \mathrm{~s}^{-1}$. Due to the deep inactive state $\mathrm{S}_{\text {off2 }}$, the transition rates between $\mathrm{S}_{\text {off } 2}$ and $\mathrm{S}_{\text {off1 }}$ are smaller than those between $\mathrm{S}_{\text {off1 }}$ and $\mathrm{S}_{\mathrm{rec}}$. We set $\lambda_{\text {off } 2}=0.006 \mathrm{~s}^{-1}$ and $\lambda_{\mathrm{on} 2}=0.002 \mathrm{~s}^{-1}$.

If we set $\lambda_{\text {off } 1}^{\text {rel }}=\lambda_{\text {off } 1}^{\text {rec }}=\lambda_{\text {off } 1}$, the ON-state dwell time can be theoretically reduced to

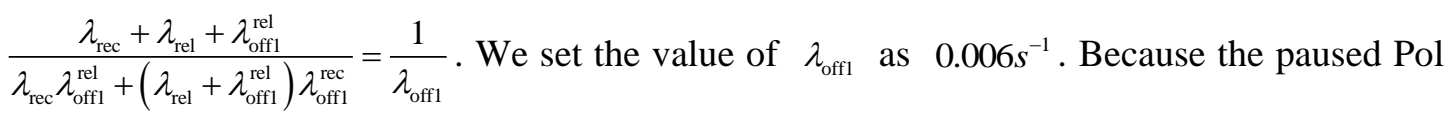
II is more stable $(38,51)$, we suppose $\lambda_{\text {off } 1}^{\text {rec }}>\lambda_{\text {off1 }}^{\text {rel }}$ and set $\quad \lambda_{\text {off } 1}^{\text {rec }}=0.009 s^{-1}, \quad \lambda_{\text {off } 1}^{\text {rel }}=0.002 s^{-1}$.

The promoter-proximal pausing timescale is about a minute level (97). We assume that the fluctuating range of the Pol II pause release rate is from $\lambda_{\text {rel, } \min }=0.008 s^{-1}$ to $\lambda_{\text {rel, } \max }=0.025 s^{-1}$. In addition, we assume that the fluctuating range of the Pol II recruitment rate is from $\lambda_{\text {rec, } \min }=0.009 s^{-1}$ to $\lambda_{\text {rec }, \max }=0.024 s^{-1}$

\section{Transcriptional elongation}

DNAs are transcribed at a speed of about $10-100 \mathrm{bp} / \mathrm{s}$ (116) across organisms and conditions. Many methods have measured that in Drosophila, the elongation rate is 
$1.1-1.5 \mathrm{~kb} / \mathrm{min}$, i.e., $\sim 25 \mathrm{bp} / \mathrm{s}$ (99-102). In addition, recent studies have suggested that in the Drosophila embryo, the Pol II elongation rate is $\sim 40 \mathrm{bp} / \mathrm{s}$ (117), approximately twice as the previously estimated value. These facts indicate that the rate of elongation is variable in different developmental stages. For simplicity, we consider that the Pol II elongation rate $r_{\mathrm{E}}$ is about $30 \mathrm{bp} / \mathrm{s}$.

In Drosophila, the size of the averagely predicted mRNA transcripts is $3058 \mathrm{bp}$ (103). We let the gene body's length $L_{\mathrm{E}}$ be roughly $3000 \mathrm{bp}$. Therefore, the transcriptional elongation time $\tau_{\mathrm{E}}$ is about $100 s$. Note that the $L_{E}$ here is different from genome length $L . L_{E}$ is the length of the gene to be transcribed after the pause release of the Pol II, which is independent of $L$ in simulations. 


\section{J Experimental confirmations and model predictions}

Our model studies verify multiple experimental results and make some experimentally testable predictions. Here we summarize and discuss a few of the predictions in details.

\section{J1 Upstream E-P Maxwell-Boltzmann distribution}

\section{E-P 3D spatial distance and its Maxwell-Boltzmann distribution}

In the main text, we set $k_{\mathrm{EP}}=0.1$ and $d_{\mathrm{G}}=50$, corresponding to $\Theta=0.183$. Both theoretical derivations and numerical simulations verify that the E-P distance follows a Maxwell-Boltzmann distribution with the mean of $\sim 0.3 \mu \mathrm{m}$, which nearly equals to $0.34 \mu \mathrm{m}$ of PP7 reporter gene in Drosophila (37) and Sox2 gene in ESCs (72). Nevertheless, the spatial distance between cis-regulatory elements and eve locus in Drosophila (82) is notably smaller $(\sim 0.2 \mu \mathrm{m})$ than that in our model. This difference may come from the fact that eve locus contains multiple enhancers, resulting in a compact globular structure with a spatial scale $\sim 0.2 \mu \mathrm{m}$.

Importantly, the work of (72) clearly showed the histogram of 3D separation distance between Sox 2 and its essential enhancer in living ESCs. Upon Maxwell-Boltzmann distribution fitting, the E-P distances fit well with the parameter $\Theta$ of 0.195 , suggesting the validity of theoretical distribution and parameter selection (SI Fig. 10B).

\section{E-P encounter probability and effective density}

Based on the theoretically-derived Maxwell-Boltzmann distribution, we can calculate the relationship between encounter probability and mean physical distance $\left(\left\langle d_{\mathrm{s}}\right\rangle\right)$. The log-log plot (SI Fig. 10E, left) shows that encounter probability is monotonically decreasing with the spatial distance following a power-law behavior, in accordance with the experimental results for consecutive and nonconsecutive TAD borders in Drosophila (113). In fact, in that reference, the interaction between TAD borders was built through some proteins (such as cohesin), similar to the E-P communication. Furthermore, the encounter probability is decreasing with E-P genomic distance and even unchanged for larger $d_{\mathrm{G}}$. For small $d_{\mathrm{G}}$, the data represent a powerlaw fitting (SI Fig. 10E, right). This finding is almost consistent to the result in (113).

On the other hand, we draw the effective density or concentration for variable radius $R$ (SI 
Fig. 10F). There is a plateau below $R \sim 0.2 \mu m$, which is supportive to the experimental finding of (82). Besides, the additional genomic separation between E5 and the promoter (82), corresponding to the increment of $d_{\mathrm{G}}$, results in an overall decrease in effective density (SI Fig. 10F, right).

\section{J2 Power-laws for transcriptional bursting kinetics}

\section{E-P communication strength}

E-P communication strength $k_{\mathrm{EP}}$ is a key parameter in the regulation of transcriptional bursting. In physics, $k_{\mathrm{EP}}$ is merely a quantity used to measure the E-P communication intensity. The $k_{\mathrm{EP}}$ can represent distinctive enhancers or external stimuli that can influence E$\mathrm{P}$ communication. For example, it was found that transcriptional burst size and traveling ratio increase during estradiol-mediated erythroid maturation (40, 53). These experimental phenomena are consistent with our model prediction that the increase $k_{\mathrm{EP}}$ can boost both burst size and burst frequency.

In fact, we have found that the $\log _{10}$ MBS and $\log _{10}$ BF respectively obey an approximately linear relation with respect to $\log _{10} k_{\mathrm{EP}}$. In the erythroid maturation experiment (40), if we use the erythroid maturation time after adding the hormone to reflect the E-P communication strength $k_{\mathrm{EP}}$, burst size of $\beta$-globin gene can fit well with a power-law function. In the work of (39), the researchers studied the effect of five distinctive enhancers on transcriptional bursting in living Drosophila embryos. We find that the burst size and burst frequency as well as transcriptional output (SI Figs. 16A-B) exhibit power-law-like behaviors when the enhancer's strength increases in an equidistant interval on a logarithmic scale. In another example, higher average c-Fos mRNA levels were induced with increasing zinc concentration (118). If taking the experimental data in the logarithmic sense, we find a linear relation between logarithmic mean mRNA expression and logarithmic $k_{\mathrm{EP}}$ representing zinc concentration (SI Fig. 16C).

\section{E-P genomic distance}

The E-P genomic distance $d_{\mathrm{G}}$ is also a key parameter in controlling transcriptional bursting. A support for this is clearly shown in the pivotal work of $(39,119)$, wherein the authors monitored the expression level of distinctive E-P distance. The burst size and frequency 
reduce when the E-P genomic distance becomes larger in sna shadow enhancer and synthetic MS2 reporter gene in Drosophila. These experimental phenomena are in accord with the results predicted by our model.

It was theoretically shown that for a smaller E-P genomic distance, the $\log _{10}$ MBS and $\log _{10} \mathrm{BF}$ respectively obey an approximately linear relation with respect to $\log _{10} d_{\mathrm{G}}$. In the work of (120), the investigators plotted the average mRNA expression level in MSCs, which approximately equals to the burst size multiplied by the burst frequency, as a function of the contact probability, which can be regarded as negative linearity over a small distance. Interestingly, the logarithmic mean mRNA expression level follows a linear relation with the logarithmic contact probability, which is consist with the prediction of our model (SI Fig. S16D). Also, the mean mRNA expression level monotonically decreased with increasing E-P genomic distance in MSCs, implying a power-law behavior (120). Importantly, the transcription level may not drop to zero but remain at a low level for larger $d_{G}$ (120).

\section{J3 E-P communication mainly regulates burst frequency}

In Section G, by theoretically calculating the values of $S_{\mathrm{BS}} / S_{\mathrm{BF}}$ and $S_{\mathrm{BS}}^{\mathrm{E}} / S_{\mathrm{BF}}^{\mathrm{E}}$, we have shown that E-P communication mainly regulates burst frequency instead of burst size.

The early studies about cell-to-cell variability indicated that the enhancer regulates the probability of transcriptional bursting, rather than the transcriptional level $(121,122)$. The core promoter determines burst size but enhancers increase bursting frequency (75). Recent experiments such as $\beta$-globin enhancer in the murine erythroblast cell during erythroid maturation (40), different developmental enhancers in Drosophila embryos (39), and transgene experiment in mouse embryonic stem cells (120), also verified this phenomenon.

Besides, transcriptome-wide inference in mouse embryonic stem cells and primary mouse fibroblasts has shown the important role of E-P communication in controlling burst frequency $(18,123)$. 


\section{K Supplementary figures}
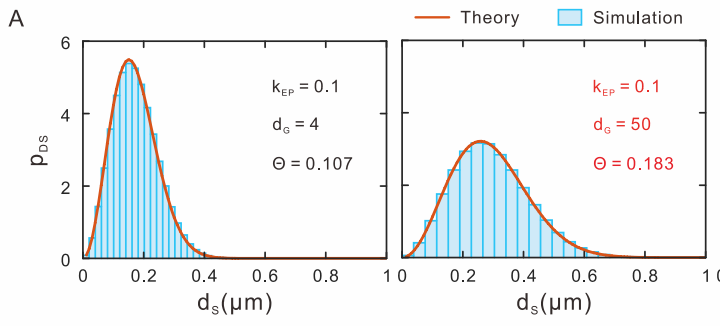

C
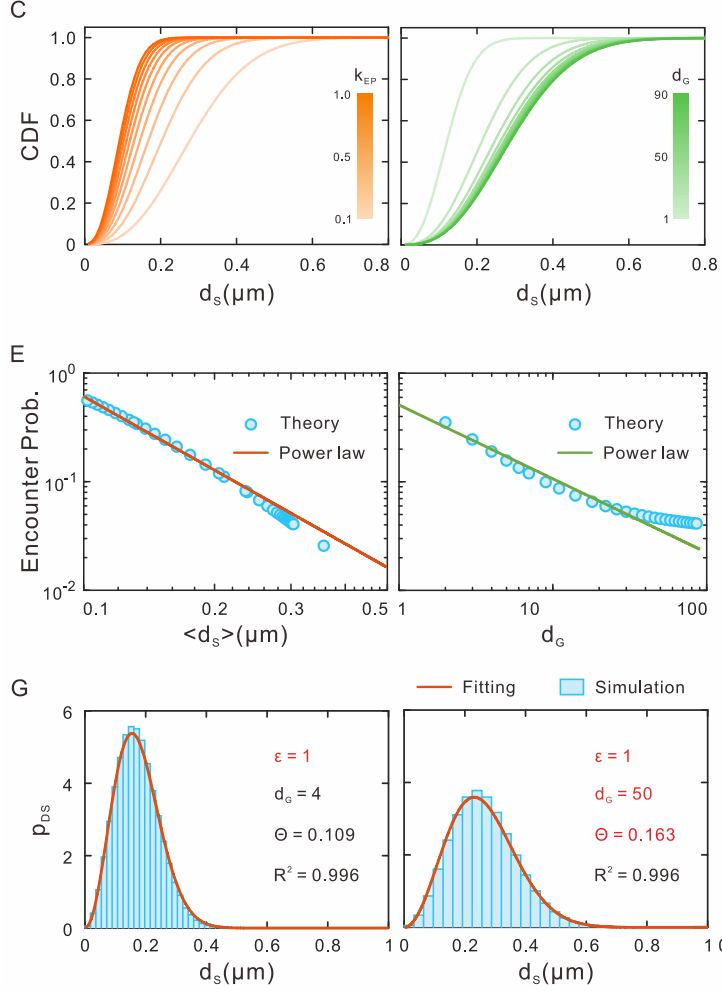
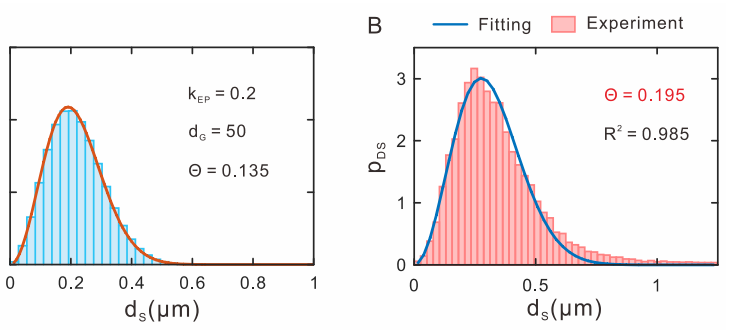

D
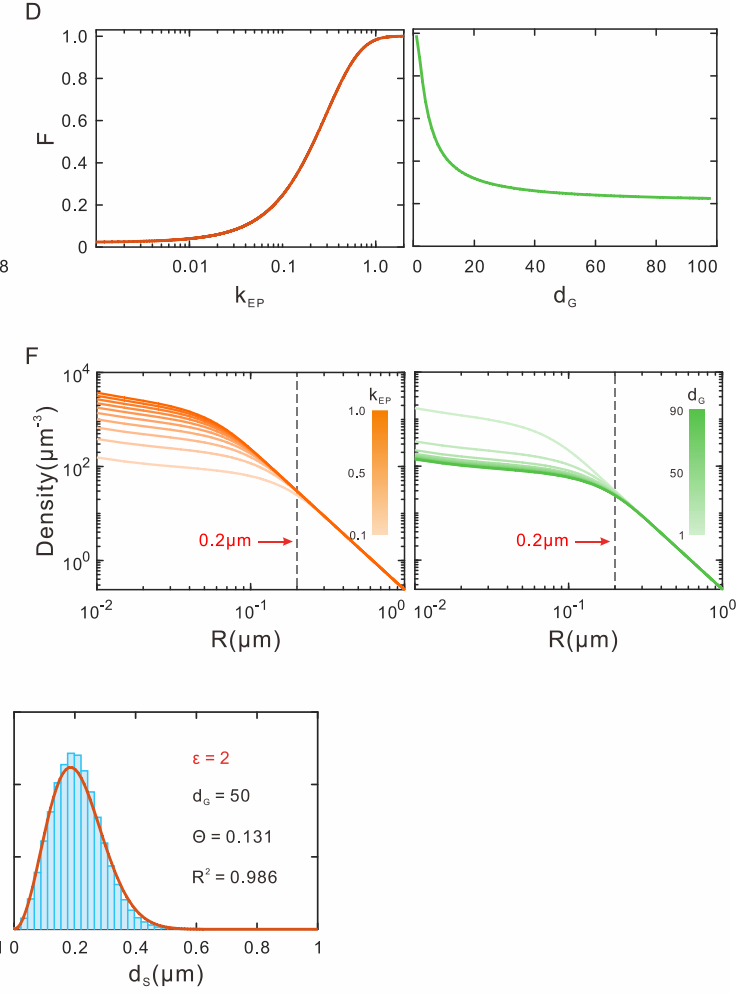

SI Figure 10. Distributions and properties of upstream E-P spatial distance. (A) Influence of $k_{\mathrm{EP}}$ and $d_{\mathrm{G}}$ on the distribution of E-P spatial distance (Maxwell-Boltzmann distribution, Eq. (E14) and (E15)) where red lines represent theoretical results, histograms represent numerical results. (B) Experimental measurement of 3D spatial distance between Sox2 and the SCR enhancer in living ESCs (72). The blue line is obtained by fitting the Maxwell-Boltzmann distribution. (C) Cumulative density function (CDF) of E-P spatial distance (Eq. (F2)) for different $k_{\mathrm{EP}}$ (left, fixed $d_{\mathrm{G}}=50$ ) and $d_{\mathrm{G}}$ (right, fixed $k_{\mathrm{EP}}=0.1$ ). (D) $F\left(k_{\mathrm{EP}}, d_{\mathrm{G}}, d_{\mathrm{S}}\right)$ as a function of $k_{\mathrm{EP}}$ (left, fixed $d_{\mathrm{G}}=50$ and $d_{\mathrm{S}}=\varepsilon_{1 / 2}$ ) and $d_{\mathrm{G}}$ (right, fixed $k_{\mathrm{EP}}=0.1$ and $\left.d_{\mathrm{S}}=\varepsilon_{1 / 2}\right)$. (E) Encounter probability as a function of mean spatial distance $\left\langle d_{\mathrm{S}}\right\rangle$ (left) and $d_{\mathrm{G}}$ 
(right). Blue points are obtained by theoretical calculation, whereas solid lines represent powerlaw fitting. Only the points with $d_{\mathrm{G}}<60$ are fitted by power-law. (F) Effective density or concentration of E-P communication as a function of the averaging volume (the sphere of radius $R$ ) for different $k_{\mathrm{EP}}$ (left, fixed $d_{\mathrm{G}}=50$ ) and $d_{\mathrm{G}}$ (right, fixed $k_{\mathrm{EP}}=0.1$ ). (G) Influence of Lennard-Jones (LJ) potential well depth $\varepsilon$ and $d_{\mathrm{G}}$ on probability density function of $d_{\mathrm{S}}$ where histograms represent numerical results and red lines represent fitting results by MaxwellBoltzmann distribution (parameter is $\Theta$ ). Other parameters are the default values as in SI Table 2. 
A

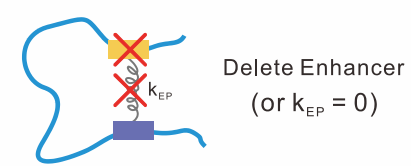

$\mathrm{F}$

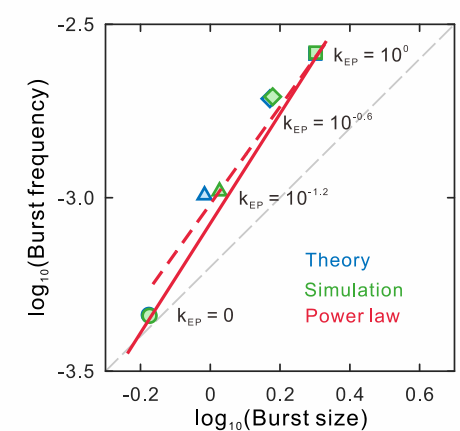

B

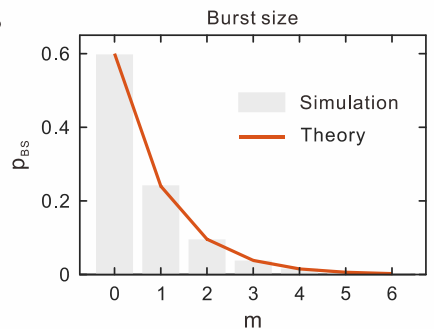

$\mathrm{D}$

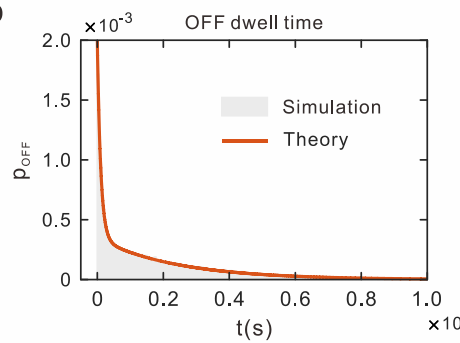

C $\quad \times 10^{-4} \quad$ Cycle time

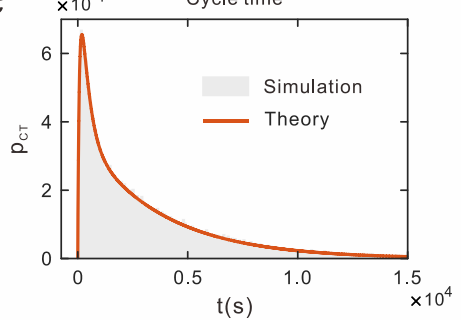

$\mathrm{E}$

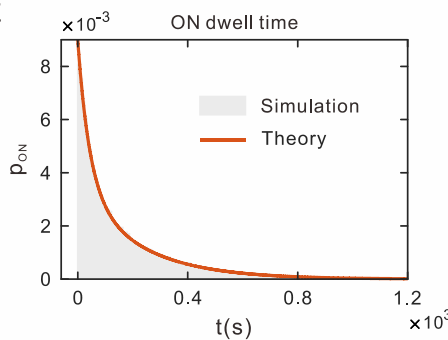

SI Figure 11. Effect of enhancer deletion on transcriptional bursting, corresponding to the case where only downstream transcriptional bursting is considered. (A) Schematic for enhancer deletion (i.e., $\left.k_{\mathrm{EP}}=0\right)$. In this case, the transcriptional burst rates are always minimums. (B-E) Probability distributions of burst size (B), cycle time (C), OFF dwell time (D) and $\mathrm{ON}$ dwell time (E) when $k_{\mathrm{EP}}=0$. The red solid lines are drawn based on Eq. (E40) for (B), Eq. (E58) for (C), Eq. (E55) for (D) and Eq. (E50) for (E), representing theoretical results. The histograms represent the results obtained by numerical simulations. (F) Influences of different values of $k_{\mathrm{EP}}$ on $\log _{10}(\mathrm{MBS})$ and $\log _{10}(\mathrm{BF})$. The red dashed line represents the approximate linear relationship between $\log _{10}(\mathrm{MBS})$ and $\log _{10}(\mathrm{BF})$ without considering enhancer deletion. And this line's slope, obtained by calculating the ratio of slope (Eq. (G1)) between $\log _{10}(\mathrm{MBS})$ and $\log _{10}\left(k_{\mathrm{EP}}\right) \quad\left(\mathrm{Eq} . \quad(\mathrm{F} 14), \quad S_{\mathrm{BS}}=0.23\right.$ ) over the slope between $\log _{10}(\mathrm{BF})$ and $\log _{10}\left(k_{\mathrm{EP}}\right)$ (Eq. (F17), $S_{\mathrm{BS}}=0.33$ ), is 1.40 . The red solid line represents the approximate linear relationship between $\log _{10}(\mathrm{MBS})$ and $\log _{10}(\mathrm{BF})$ when $k_{\mathrm{EP}}=0$ and the line slope is 1.56 . The dashed gray line is the indicator of the slope equaling 1 . Other parameters are the default values as in SI Table 2. 
A
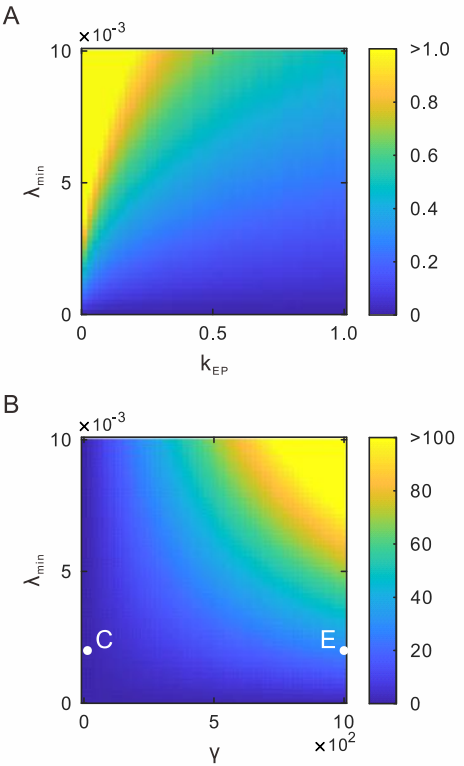

C
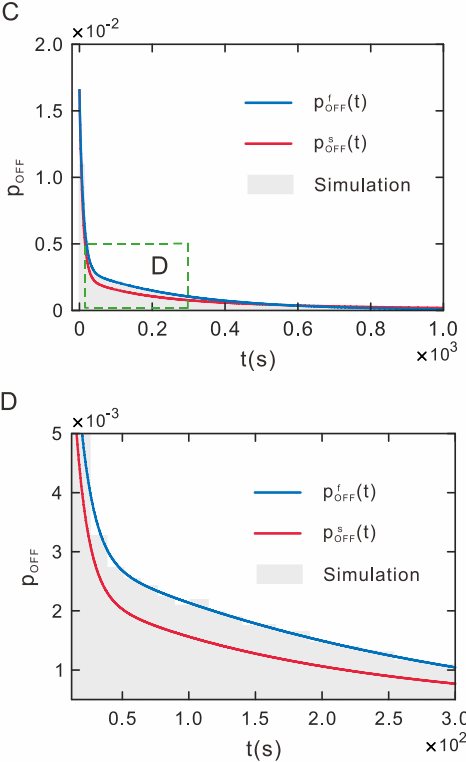
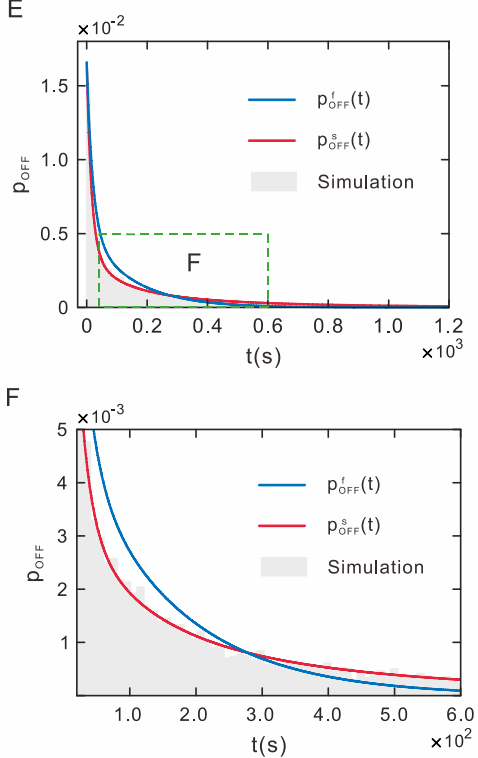

SI Figure 12. The effect of upstream and downstream timescales on downstream distributions, where OFF distribution is taken as an example for demonstration. (A) The maximum achievable ratio of upstream and downstream timescales $\omega$ in Eq. (E1) is a function of minimum state switching rates $\lambda_{\min }$ and E-P communication strength $k_{\mathrm{EP}}$. (B) The $\omega$ is a function of minimum transcriptional rate $\lambda_{\min }$ and drag coefficient $\gamma$. (C-F) Blue solid lines representing the OFF PDFs are drawn by Eq. (E3), whereas red solid lines by Eq. (E5). The histograms represent the results obtained by numerical simulations. (C-D) Upstream chromatin fluctuations are much faster compared with the downstream state switching rates. Parameter values are set as $\gamma=0.1, \lambda_{\text {on } 1 \text { min }}=0.002, \quad \lambda_{\text {on1, max }}=0.05, \quad \lambda_{\text {off } 2}=0.06, \quad \lambda_{\text {on } 2}=0.02$. (E-F) Upstream chromatin fluctuations are slow. Parameter values are set as $\gamma=1000$, $\lambda_{\text {on } 1 \text { min }}=0.002, \lambda_{\text {on }, \text { max }}=0.05, \quad \lambda_{\text {off } 2}=0.02, \quad \lambda_{\text {on } 2}=0.02$. Other parameters are the default values as in SI Table 2. 


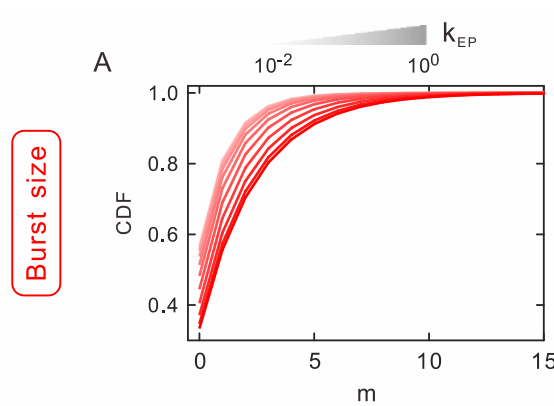

B

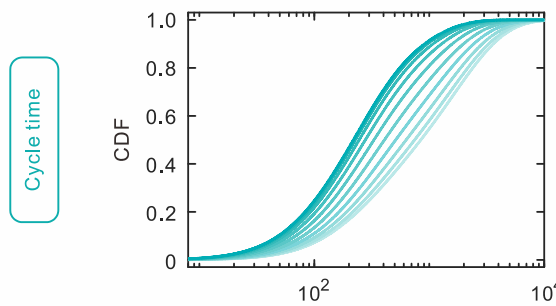

$t(s)$

C

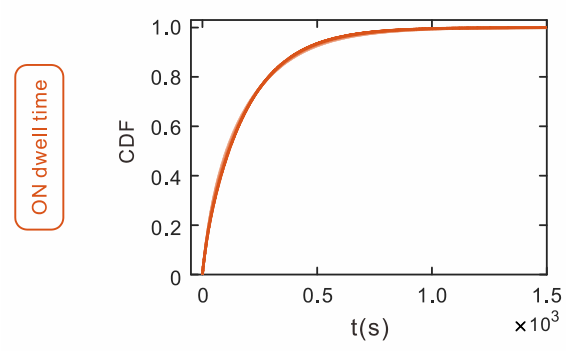

$\mathrm{D}$

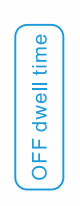

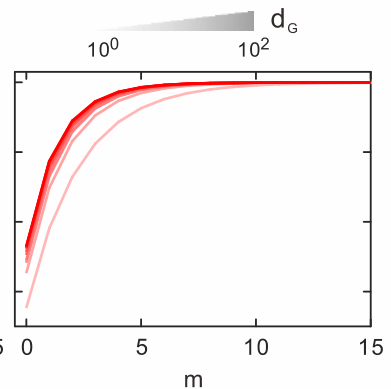

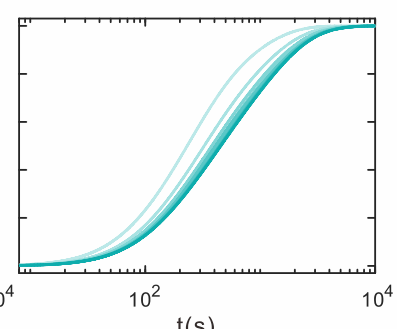

$\mathrm{t}(\mathrm{s})$

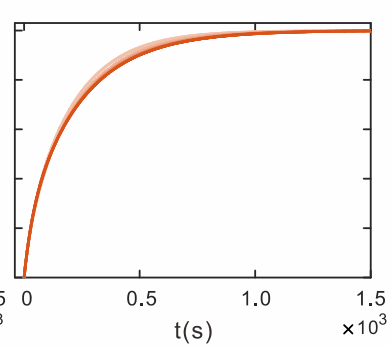

$\mathrm{t}(\mathrm{s})$

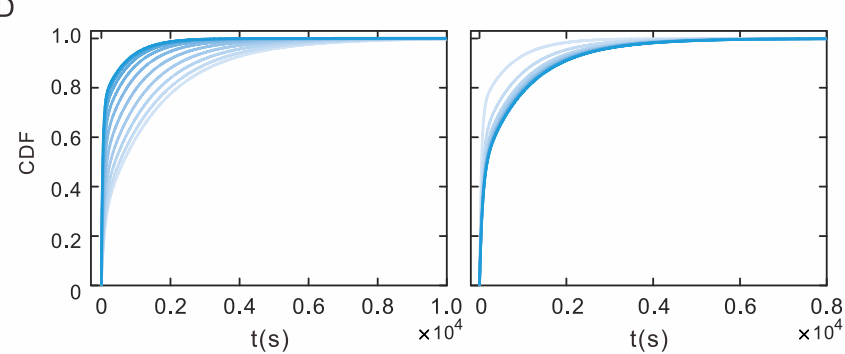

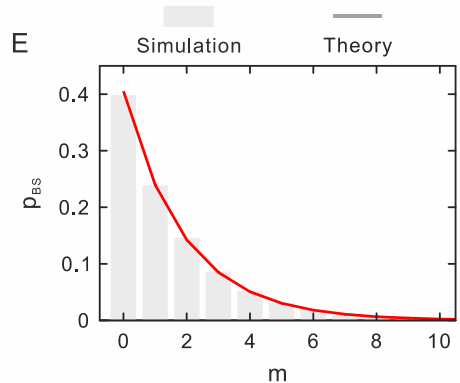

$\mathrm{F}$

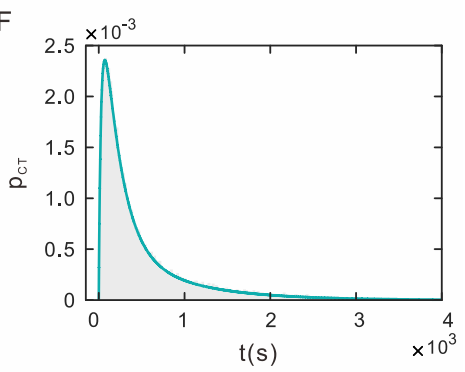

G

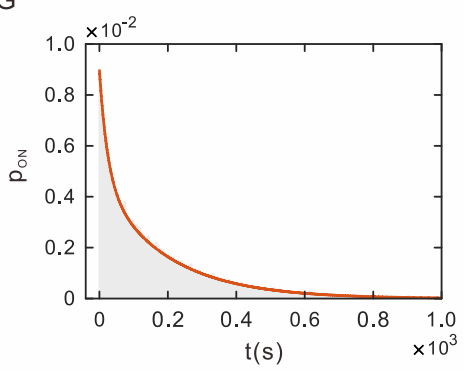

$\mathrm{H}$

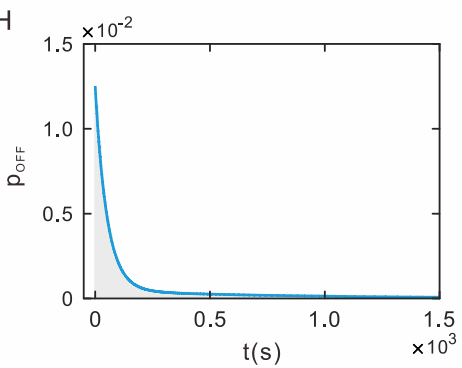

SI Figure 13. Distributions of burst size, cycle time, ON time and OFF time. (A-D) CDF

(The corresponding PDFs or PMF are shown in Eq. (E6)) of burst size (A), cycle time (B), ON time (C) and OFF time (D) for different $k_{\mathrm{EP}}$ (left, fixed $d_{\mathrm{G}}=50$ ) and $d_{\mathrm{G}}$ (right, fixed $\left.k_{\mathrm{EP}}=0.1\right) .(\mathrm{E}-\mathrm{H})$ Probability distributions of burst size $(\mathrm{E})$, cycle time $(\mathrm{F})$, OFF dwell time $(\mathrm{G})$ and $\mathrm{ON}$ dwell time $(\mathrm{H})$ where numerical simulation results correspond to grey histograms whereas analytical results to solid lines. Parameter values in $(\mathrm{E}-\mathrm{H})$ are set as $k_{\mathrm{EP}}=10^{-0.6}$, $d_{\mathrm{G}}=50$. Other parameters are the default values as in SI Table 2. 


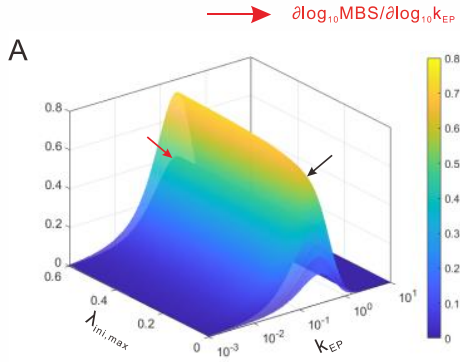

C

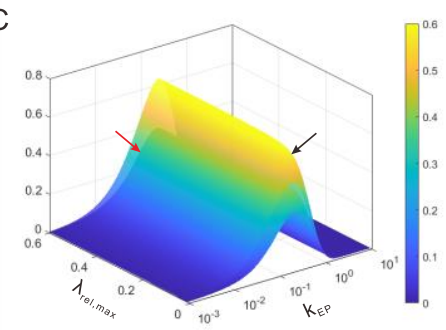

F

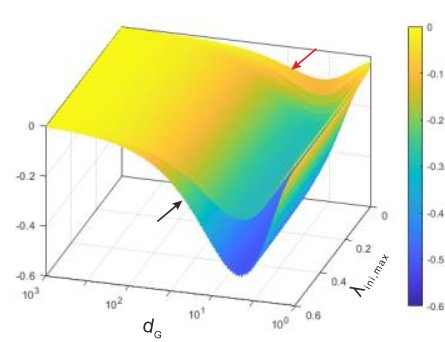

$\mathrm{H}$

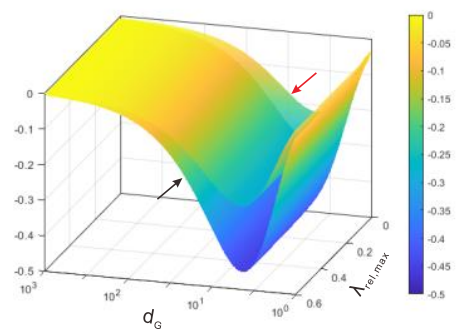

B

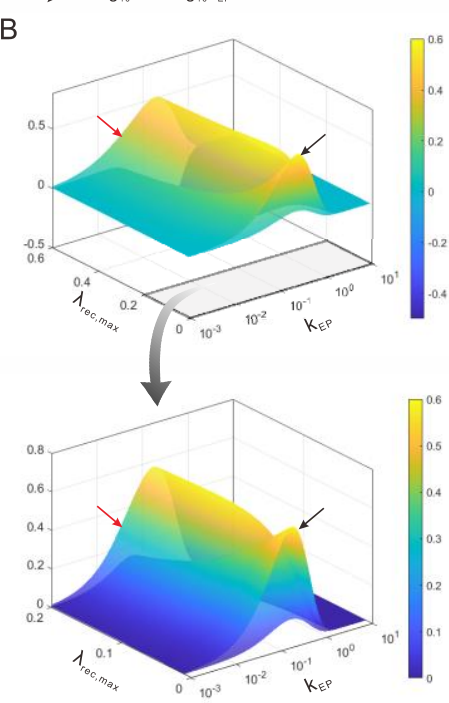

$\longrightarrow \log _{10} \mathrm{BF} / 2 \log _{10} \mathrm{~d}_{6}$

G
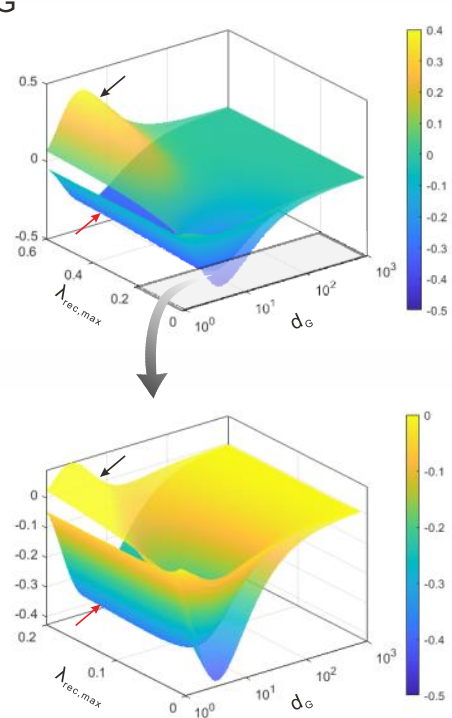

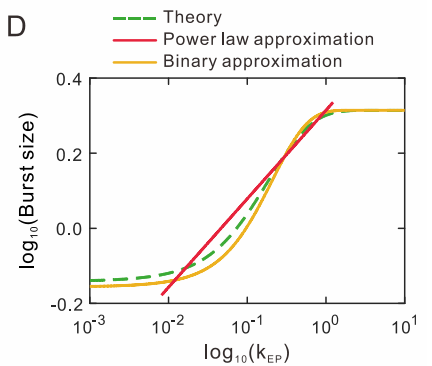

$\mathrm{E}$

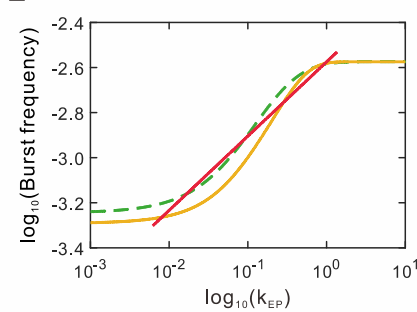

I --- Theory

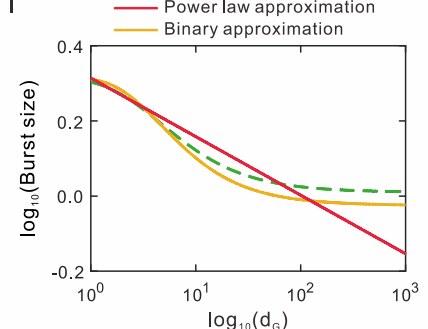

$\mathrm{J}$

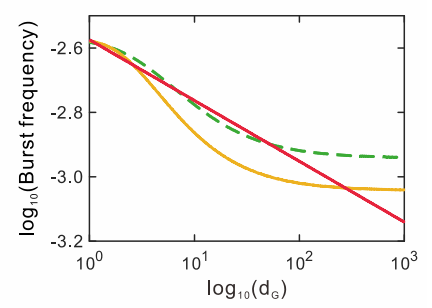

SI Figure 14. Rationality and validity analysis of power-law approximations for transcriptional bursting kinetics. (A-C) Derivatives $\frac{\partial \log _{10} \mathrm{MBS}}{\partial \log _{10} k_{\mathrm{EP}}} \quad$ (Eq. (F10)) and $\frac{d \log _{10} \mathrm{BF}}{d \log _{10} k_{\mathrm{EP}}}$ (Eq. (F12)) as the functions of parameters $k_{\mathrm{EP}}$ and $\lambda_{\mathrm{in}, \max }(\mathrm{A}), \lambda_{\mathrm{rec}, \max }$ (B), $\lambda_{\text {rel,max }}(C)$. The bottom of (B) is a magnified display of a portion of the top of (B). (D-E) $\log _{10}$ MBS and $\log _{10}$ BF as the functions of $\log _{10}\left(k_{\mathrm{EP}}\right)$, where green dashed lines represent theoretical prediction (Eq. (E7)), orange solid lines represent binary approximation (Eq. (E7)), and red solid lines represent power-law approximation (Eq. (F15)). (F-H) Derivatives 
$\frac{\partial \log _{10} \mathrm{MBS}}{\partial \log _{10} d_{\mathrm{G}}}$ and $\frac{d \log _{10} \mathrm{BF}}{d \log _{10} d_{\mathrm{G}}}$ (Eq. (F20)) as the functions of parameter $d_{\mathrm{G}}$ and $\lambda_{\text {ini,max }}$ (F), $\lambda_{\text {rec,max }}(\mathrm{G}), \lambda_{\text {rel,max }}(\mathrm{H})$. The bottom of $(\mathrm{G})$ is a magnified display of a portion of the top of $(\mathrm{G})$.

(I-J) $\log _{10}$ MBS and $\log _{10}$ BF as the functions of $\log _{10}\left(d_{\mathrm{G}}\right)$, where green dashed lines represent theoretical prediction (Eq. (E7)), orange solid lines represent binary approximation (Eq. (E7)), and red solid lines represent power-law approximation (Eq. (F18)). Other parameters are set as the default values in SI Table 2. 
A
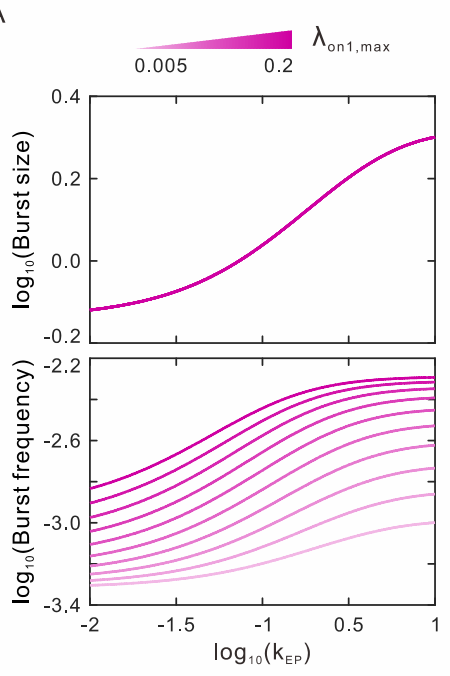

D
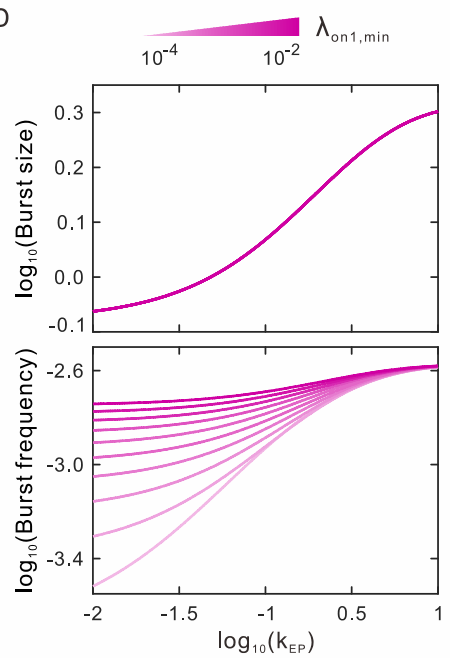

G

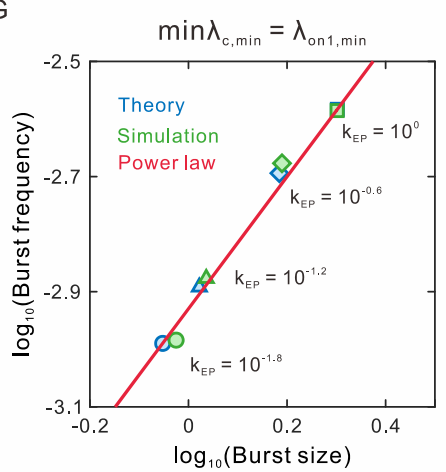

B

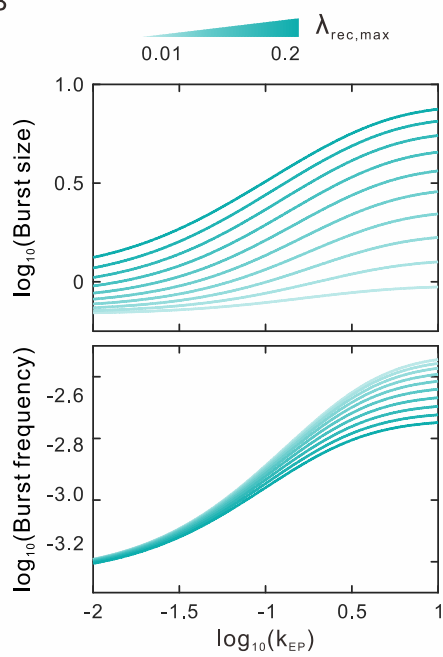

$\mathrm{E}$
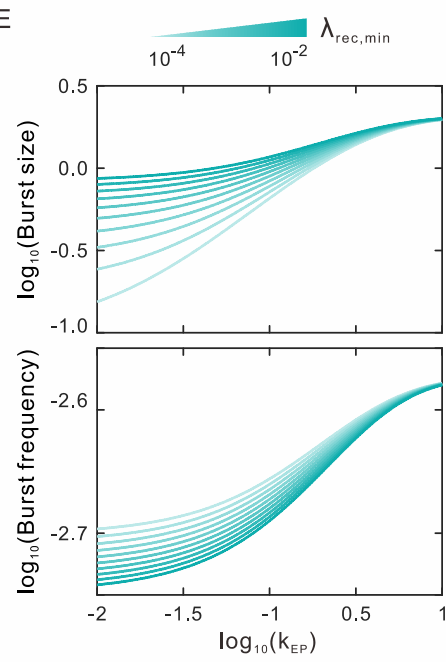

$\mathrm{H}$

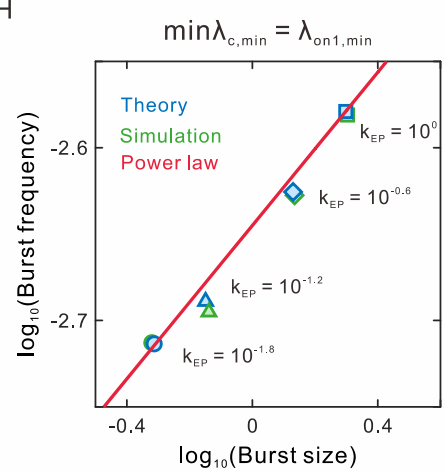

C

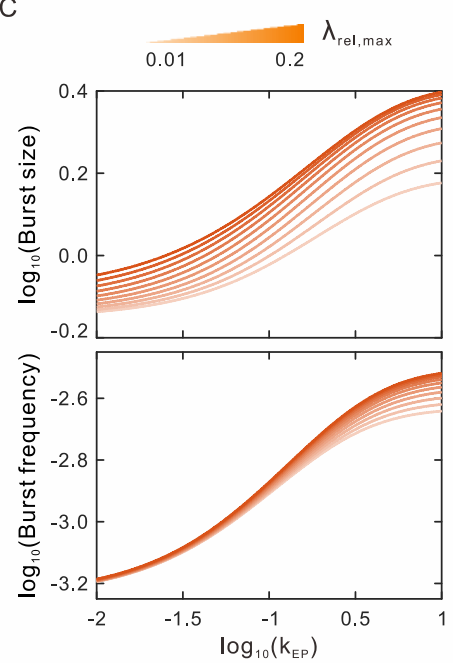

F
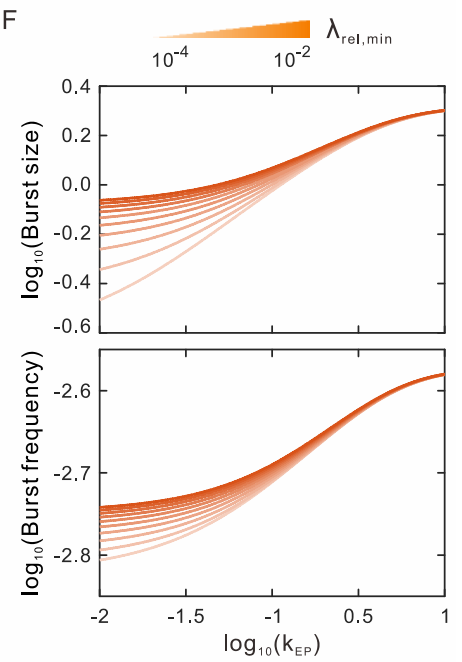

I

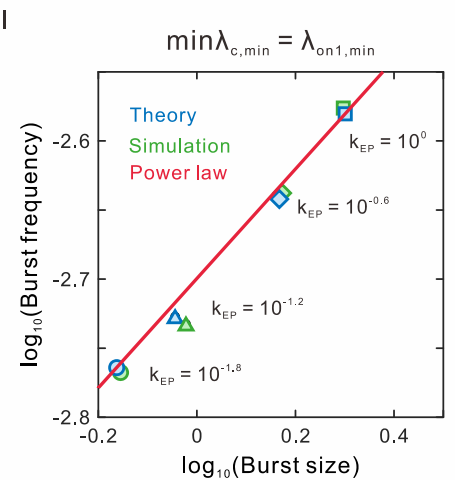

SI Figure 15. Effects of model parameters and upstream and downstream scales on burst size and burst frequency on logarithmic scale. (A-C) Influences of model parameters $\lambda_{\text {on1,max }}$
(A), $\quad \lambda_{\text {rec, } \max }$
(B), $\lambda_{\text {rel, max }}$
(C) on burst size (top) and burst frequency (bottom), where other

parameter values are the same as the default settings in SI Table 3. (D-F) Influences of $\min \lambda_{c, \text { min }}$ on burst size (top) and burst frequency (bottom). $\min _{c}\left\{\lambda_{c, \text { min }}\right\}=\lambda_{\text {onl, min }}$ in (D) with 
fixed $\lambda_{\text {rec, min }}=0.01, \quad \lambda_{\text {rel, min }}=0.01 . \min _{c}\left\{\lambda_{c, \text { min }}\right\}=\lambda_{\text {rec, } \min }$ in (E) with fixed $\lambda_{\text {on } 1 \text { min }}=0.01$, $\lambda_{\text {rel,min }}=0.01 . \min _{c}\left\{\lambda_{c, \text { min }}\right\}=\lambda_{\text {rel,min }}$ in (F) with fixed $\lambda_{\text {on } 1, \text { min }}=0.01, \quad \lambda_{\text {rec, min }}=0.01$. Other parameter values are the same as the default settings in SI Table 3. (G-I) Influences of different values of $k_{\mathrm{EP}}$ on $\log _{10}(\mathrm{MBS})$ and $\log _{10}(\mathrm{BF})$. The red solid lines represent the approximate linear relationship between $\log _{10}(\mathrm{MBS})$ and $\log _{10}(\mathrm{BF})$. Parameter values are set as $\lambda_{\text {on } 1 \text { min }}=0.004, \lambda_{\text {rec, } \text { min }}=0.01, \quad \lambda_{\text {rel, min }}=0.01$ in $(G), \quad \lambda_{\text {on1, min }}=0.01, \quad \lambda_{\text {rec, } \text { min }}=0.004, \quad \lambda_{\text {rel, min }}=0.01$ in $(\mathrm{H})$ and $\lambda_{\text {on1,min }}=0.01, \lambda_{\text {rec, } \text { min }}=0.01, \lambda_{\text {rel,min }}=0.004$ in (I). Other parameter values are the default values as in SI Table 2. 
A

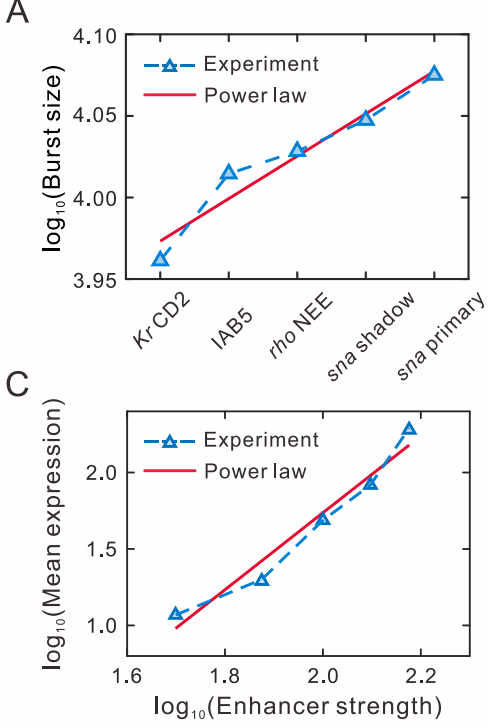

B
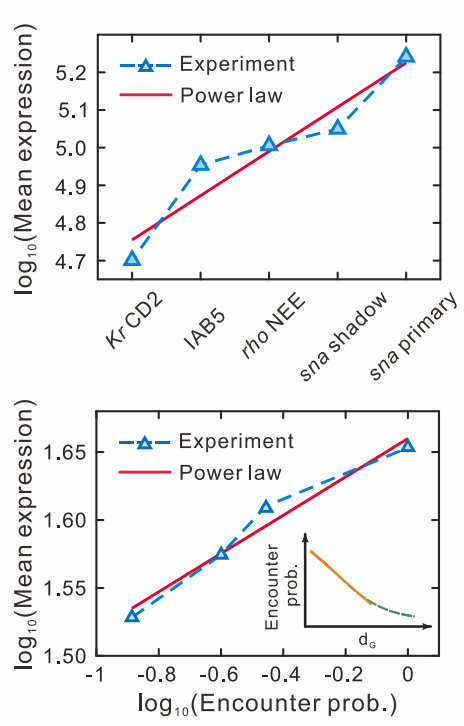

SI Figure 16. Experimental data verify the validity of power-law behaviors on burst size and burst frequency as well as mean expression level. (A-B) Plot of experimental data from (39). The labels of the $x$-axis represent the sna shadow, sna primary, rho NEE, IAB5, and $K r$ CD2 enhancers, respectively. (C) Log-log plot of experimental data from (118). (D) Log-log plot of experimental data from (120). Insert figure shows the log-log relationship between $d_{\mathrm{G}}$ and encounter probability. The yellow line indicates an approximate power-law relationship for smaller $d_{\mathrm{G}}$. 

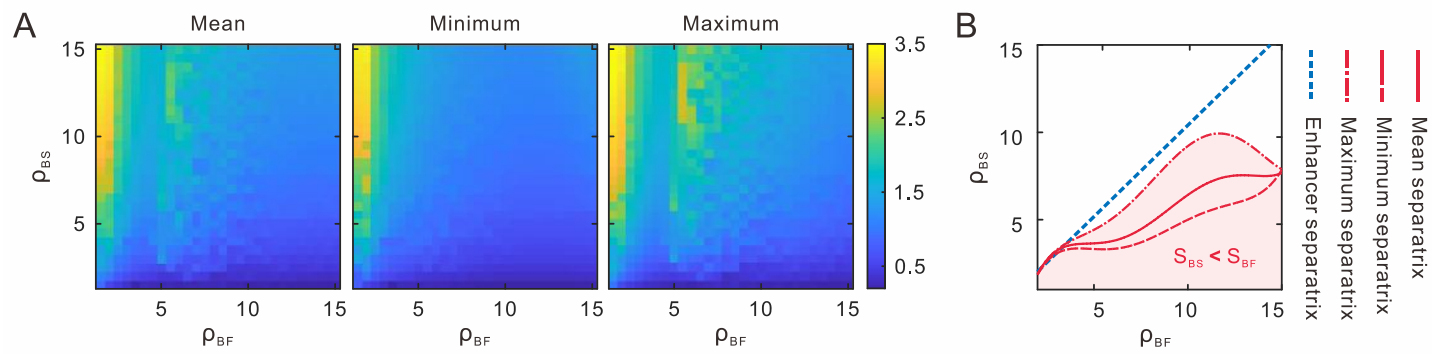

SI Figure 17. Effects of model parameters and upstream and downstream scales on ratio

$S_{\mathrm{BF}} / S_{\mathrm{BS}}$ in the $\left(\rho_{\mathrm{BF}}, \rho_{\mathrm{BS}}\right)$ plane. (A-C) Mean slope ratio for (A), minimum slope ratio for (B) and maximum slope ratio for (C) are obtained after the $S_{\mathrm{BF}} / S_{\mathrm{BS}}$ is calculated according to Eq. (F17) and Eq. (F14). (D) A phase diagram illustrates the size boundaries of the slopes of burst size and burst frequency in the $\left(\rho_{\mathrm{BF}}, \rho_{\mathrm{BS}}\right)$ plane. The red lines are the separatrixes of data in (A). The blue dashed line is the separatrix in the case of enhancer deletion. Other parameter values are the default values in SI Table 2. 

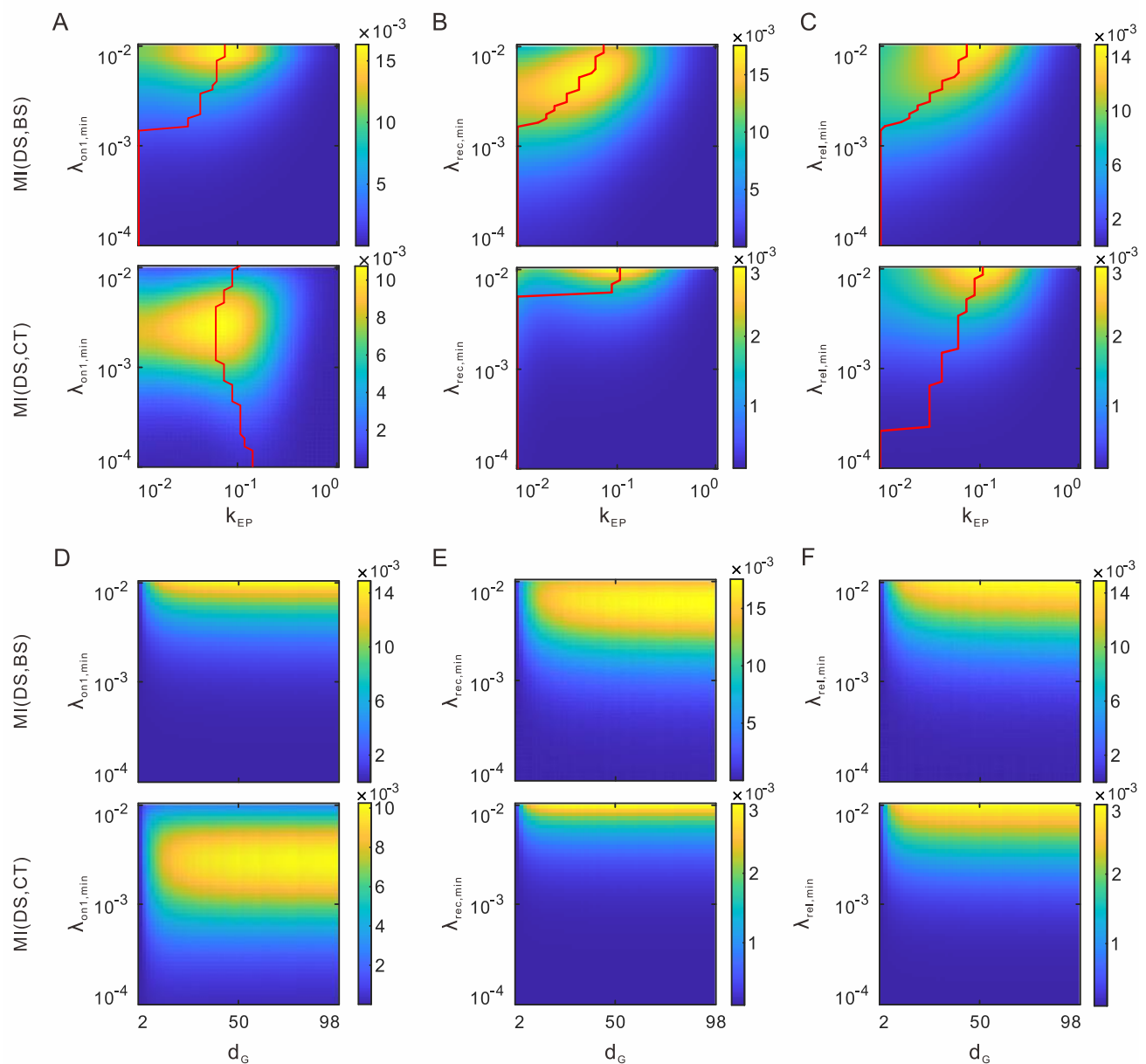

SI Figure 18. Influence of model parameters on mutual information. (A-C) Heatmap shows the joint effects of communication strength $k_{\mathrm{EP}}$ and minimum rate $\lambda_{\text {on1,min }}$ for fixed $\lambda_{\text {rec, min }}=0.01, \lambda_{\text {rel, min }}=0.01$ in (A), $\lambda_{\text {rec,min }}$ for fixed $\lambda_{\text {onl,min }}=0.01, \quad \lambda_{\text {rel, min }}=0.01$ in $(B)$, $\lambda_{\text {rel,min }}$ for fixed $\lambda_{\text {onl,min }}=0.01, \lambda_{\text {rec, min }}=0.01$ in (C) on $\operatorname{MI}(D S, B S)$ (top) and $\operatorname{MI}(D S, C T)$ (bottom). Parameter values in (A) are the same as the default settings in SI Table 3. The red line shows the values of $k_{\mathrm{EP}}$ corresponding to the maximum mutual information. (D-F) Heatmap shows the joint effects of genomic distance $d_{\mathrm{G}}$ and minimum rate $\lambda_{\text {on1,min }}$ for fixed $\lambda_{\text {rec, } \text { min }}=0.01, \lambda_{\text {rel,min }}=0.01$ in (D), $\lambda_{\text {rec,min }}$ for fixed $\lambda_{\text {onl,min }}=0.01, \lambda_{\text {rel, min }}=0.01$ in (E), $\lambda_{\text {rel, min }}$ for fixed $\lambda_{\text {onl,min }}=0.01, \lambda_{\text {rec,min }}=0.01$ in $(\mathrm{F})$ on $\operatorname{MI}(D S, B S)$ (top) and $\operatorname{MI}(D S, C T)$ (bottom). Other parameter values are the same as the default settings in SI Table 2. 

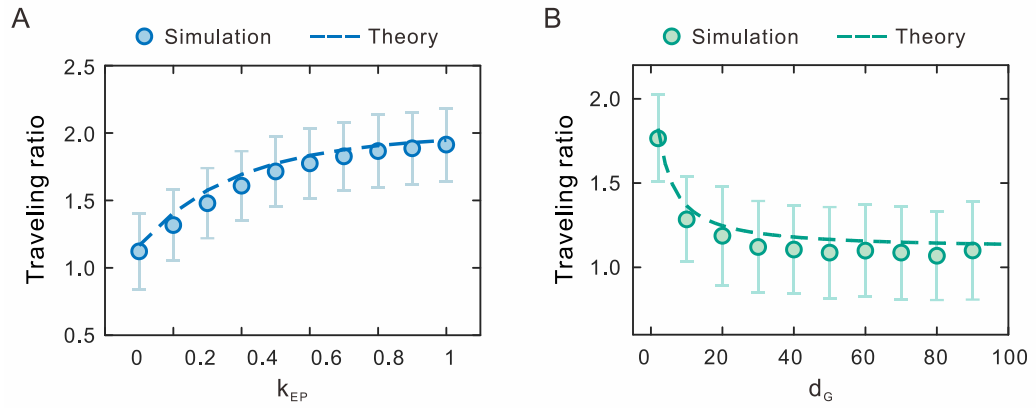

SI Figure 19. Influence of parameters $k_{\mathrm{EP}}$ (A) and $d_{\mathrm{G}}$ (B) on traveling ratio. The parameter values are set as $\lambda_{\text {on } 2}=0.003, \quad \lambda_{\text {off }}=0.006, \quad \lambda_{\text {on } 1 \text {, min }}=0.003, \quad \lambda_{\text {on } 1 \text { max }}=0.020$, $\lambda_{\text {rec, } \text { min }}=0.010, \lambda_{\text {rec,max }}=0.030, \lambda_{\text {rel,min }}=0.008, \lambda_{\text {rel,max }}=0.020, \lambda_{\text {off } 1}^{\text {rec }}=0.008, \lambda_{\text {off } 1}^{\text {rel }}=0.003$. Other parameter values are the default values as in SI Table 2. 


\section{References}

1. T. Misteli, The self-organizing genome: Principles of genome architecture and function. Cell 183, 28-45 (2020).

2. M. J. Rowley, V. G. Corces, Organizational principles of 3D genome architecture. Nat. Rev. Genet. 19, 789-800 (2018).

3. V. Sood, T. Misteli, The stochastic nature of genome organization and function. Curr. Opin. Genet. Dev. 72, 45-52 (2022).

4. J.-H. Su, P. Zheng, S. S. Kinrot, B. Bintu, X. Zhuang, Genome-scale imaging of the 3D organization and transcriptional activity of chromatin. Cell 182, 1641-1659 (2020).

5. L. J. Mateo et al., Visualizing DNA folding and RNA in embryos at single-cell resolution. Nature 568, 49-54 (2019).

6. E. H. Finn et al., Extensive heterogeneity and intrinsic variation in spatial genome organization. Cell 176, 1502-1515 (2019).

7. J. M. Luppino et al., Cohesin promotes stochastic domain intermingling to ensure proper regulation of boundary-proximal genes. Nat. Genet. 52, 840-848 (2020).

8. M. I. Robson, A. R. Ringel, S. Mundlos, Regulatory landscaping: How enhancer-promoter communication is sculpted in 3D. Mol. Cell 74, 1110-1122 (2019).

9. N. J. Fuda, M. B. Ardehali, J. T. Lis, Defining mechanisms that regulate RNA polymerase II transcription in vivo. Nature 461, 186-192 (2009).

10. A. Raj, C. S. Peskin, D. Tranchina, D. Y. Vargas, S. Tyagi, Stochastic mRNA synthesis in mammalian cells. PLoS Biol. 4, e309 (2006).

11. I. Golding, J. Paulsson, S. M. Zawilski, E. C. Cox, Real-time kinetics of gene activity in individual bacteria. Cell 123, 1025-1036 (2005).

12. D. R. Larson, D. Zenklusen, B. Wu, J. A. Chao, R. H. Singer, Real-time observation of transcription initiation and elongation on an endogenous yeast gene. Science 332, 475-478 (2011).

13. D. M. Suter et al., Mammalian genes are transcribed with widely different bursting kinetics. Science 332, 472-474 (2011).

14. J. P. Bothma et al., Dynamic regulation of eve stripe 2 expression reveals transcriptional bursts in living Drosophila embryos. Proc. Natl. Acad. Sci. U.S.A. 111, 10598-10603 (2014).

15. J. Peccoud, B. Ycart, Markovian modeling of gene-product synthesis. Theor. Popul. Biol. 48, 222234 (1995).

16. K. Tantale et al., A single-molecule view of transcription reveals convoys of RNA polymerases and multi-scale bursting. Nat. Commun. 7, 12248 (2016).

17. G. Neuert et al., Systematic identification of signal-activated stochastic gene regulation. Science 339, 584-587 (2013).

18. A. J. M. Larsson et al., Genomic encoding of transcriptional burst kinetics. Nature 565, 251-254 (2019).

19. W. J. Blake, M. KÆrn, C. R. Cantor, J. J. Collins, Noise in eukaryotic gene expression. Nature 422, 633-637 (2003).

20. A. Sanchez, H. G. Garcia, D. Jones, R. Phillips, J. Kondev, Effect of promoter architecture on the cell-to-cell variability in gene expression. PLoS Comput. Biol. 7, e1001100 (2011).

21. W. De Laat, D. Duboule, Topology of mammalian developmental enhancers and their regulatory 
landscapes. Nature 502, 499-506 (2013).

22. O. I. Kulaeva, F.-K. Hsieh, H.-W. Chang, D. S. Luse, V. M. Studitsky, Mechanism of transcription through a nucleosome by RNA polymerase II. BBA-Gene Regul. Mech. 1829, 76-83 (2013).

23. G. Li, M. Levitus, C. Bustamante, J. Widom, Rapid spontaneous accessibility of nucleosomal DNA. Nat. Struct. Mol. Biol. 12, 46-53 (2005).

24. S. R. Kassabov, B. Zhang, J. Persinger, B. Bartholomew, SWI/SNF unwraps, slides, and rewraps the nucleosome. Mol. Cell 11, 391-403 (2003).

25. T. Heist, T. Fukaya, M. Levine, Large distances separate coregulated genes in living Drosophila embryos. Proc. Natl. Acad. Sci. U.S.A. 116, 15062-15067 (2019).

26. B. Lim, T. Heist, M. Levine, T. Fukaya, Visualization of transvection in living Drosophila embryos. Mol. Cell 70, 287-296 (2018).

27. C. P. Johnstone, N. B. Wang, S. A. Sevier, K. E. Galloway, Understanding and engineering chromatin as a dynamical system across length and timescales. Cell Syst. 11, 424-448 (2020).

28. O. Shukron, D. Holcman, Transient chromatin properties revealed by polymer models and stochastic simulations constructed from Chromosomal Capture data. PLoS Comput. Biol. 13, e1005469 (2017).

29. D. Jost, C. Vaillant, Epigenomics in 3D: importance of long-range spreading and specific interactions in epigenomic maintenance. Nucleic Acids Res. 46, 2252-2264 (2018).

30. S. K. Ghosh, D. Jost, How epigenome drives chromatin folding and dynamics, insights from efficient coarse-grained models of chromosomes. PLoS Comput. Biol. 14, e1006159 (2018).

31. M. Mir et al., Dynamic multifactor hubs interact transiently with sites of active transcription in Drosophila embryos. eLife 7, e40497 (2018).

32. T. Morisaki, W. G. Müller, N. Golob, D. Mazza, J. G. McNally, Single-molecule analysis of transcription factor binding at transcription sites in live cells. Nat. Commun. 5, 4456 (2014).

33. J. Chen et al., Single-molecule dynamics of enhanceosome assembly in embryonic stem cells. Cell 156, 1274-1285 (2014).

34. J. Dufourt et al., Temporal control of gene expression by the pioneer factor Zelda through transient interactions in hubs. Nat. Commun. 9, 5194 (2018).

35. B. Alberts et al., Molecular biology of the cell (Garland Science, New York, NY, ed. 4, 2003).

36. W.-K. Cho et al., RNA Polymerase II cluster dynamics predict mRNA output in living cells. eLife 5, e13617 (2016).

37. H. Chen et al., Dynamic interplay between enhancer-promoter topology and gene activity. Nat. Genet. 50, 1296-1303 (2018).

38. W. Shao, J. Zeitlinger, Paused RNA polymerase II inhibits new transcriptional initiation. Nat. Genet. 49, 1045-1051 (2017).

39. T. Fukaya, B. Lim, M. Levine, Enhancer control of transcriptional bursting. Cell 166, 358-368 (2016).

40. C. R. Bartman, S. C. Hsu, C. C. Hsiung, A. Raj, G. A. Blobel, Enhancer regulation of transcriptional bursting parameters revealed by forced chromatin looping. Mol. Cell 62, 237-247 (2016).

41. B. T. Donovan et al., Live-cell imaging reveals the interplay between transcription factors, nucleosomes, and bursting. EMBO J. 38, e100809 (2019).

42. J. Li et al., Single-gene imaging links genome topology, promoter-enhancer communication and transcription control. Nat. Struct. Mol. Biol. 27, 1032-1040 (2020).

43. A. M. C. Gizzi et al., Microscopy-based chromosome conformation capture enables simultaneous 
visualization of genome organization and transcription in intact organisms. Mol. Cell 74, 212-222 (2019).

44. N. C. Lammers, Y. J. Kim, J. Zhao, H. G. Garcia, A matter of time: Using dynamics and theory to uncover mechanisms of transcriptional bursting. Curr. Opin. Cell Biol. 67, 147-157 (2020).

45. D. T. Gillespie, Exact stochastic simulation of coupled chemical reactions. J. Phys. Chem. 81, 2340-2361 (1977).

46. C. V. Harper et al., Dynamic analysis of stochastic transcription cycles. PLoS Biol. 9, e1000607 (2011).

47. A. Mayran et al., Pioneer and nonpioneer factor cooperation drives lineage specific chromatin opening. Nat. Commun. 10, 3807 (2019).

48. J. T. Lis, A 50 year history of technologies that drove discovery in eukaryotic transcription regulation. Nat. Struct. Mol. Biol. 26, 777-782 (2019).

49. S. Nechaev et al., Global analysis of short RNAs reveals widespread promoter-proximal stalling and arrest of Pol II in Drosophila. Science 327, 335-338 (2010).

50. L. J. Core, J. J. Waterfall, J. T. Lis, Nascent RNA sequencing reveals widespread pausing and divergent initiation at human promoters. Science 322, 1845-1848 (2008)

51. T. Henriques et al., Stable pausing by RNA polymerase II provides an opportunity to target and integrate regulatory signals. Mol. Cell 52, 517-528 (2013).

52. R. C. Wilkins, J. T. Lis, Dynamics of potentiation and activation: GAGA factor and its role in heat shock gene regulation. Nucleic Acids Res. 25, 3963-3968 (1997).

53. C. R. Bartman et al., Transcriptional burst initiation and polymerase pause release are key control points of transcriptional regulation. Mol. Cell 73, 519-532 (2019).

54. J. N. Kuehner, E. L. Pearson, C. Moore, Unravelling the means to an end: RNA polymerase II transcription termination. Nat. Rev. Mol. Cell Biol. 12, 283-294 (2011).

55. T. S. Zhou, J. J. Zhang, Analytical results for a multi-state gene model. SIAM J. Appl. Math. 72, 789-818 (2012).

56. J. Rodriguez et al., Intrinsic dynamics of a human gene reveal the basis of expression heterogeneity. Cell 176, 213-226 (2019).

57. K. S. Zaret, J. S. Carroll, Pioneer transcription factors: establishing competence for gene expression. Genes Dev. 25, 2227-2241 (2011).

58. Y. J. Li, X. H. Fu, D. P. Liu, C. C. Liang, Opening the chromatin for transcription. Int. J. Biochem. Cell Biol. 36, 1411-1423 (2004).

59. D. Yusuf et al., The transcription factor encyclopedia. Genome Biol. 13, R24 (2012).

60. J. Soutourina, Transcription regulation by the Mediator complex. Nat. Rev. Mol. Cell Biol. 19, 262274 (2018).

61. S. Schilbach et al., Structures of transcription pre-initiation complex with TFIIH and Mediator. Nature 551, 204-209 (2017).

62. A. Dvir, J. W. Conaway, R. C. Conaway, Mechanism of transcription initiation and promoter escape by RNA polymerase II. Curr. Opin. Genet. Dev. 11, 209-214 (2001).

63. K. Adelman, J. T. Lis, Promoter-proximal pausing of RNA polymerase II: emerging roles in metazoans. Nat. Rev. Genet. 13, 720-731 (2012).

64. F. X. Chen et al., PAF1 regulation of promoter-proximal pause release via enhancer activation. Science 357, 1294-1298 (2017).

65. Q. Zhou, T. Li, D. H. Price, RNA polymerase II elongation control. Annu. Rev. Biochem. 81, 119- 
143 (2012).

66. B. Lim, M. S. Levine, Enhancer-promoter communication: Hubs or loops? Curr. Opin. Genet. Dev. 67, 5-9 (2021).

67. E. E. M. Furlong, M. Levine, Developmental enhancers and chromosome topology. Science 361, 1341-1345 (2018).

68. W. Deng et al., Controlling long-Range genomic interactions at a native locus by targeted tethering of a looping factor. Cell 149, 1233-1244 (2012).

69. G. Li et al., Extensive Promoter-Centered Chromatin Interactions Provide a Topological Basis for Transcription Regulation. Cell 148, 84-98 (2012)

70. S. S. Rao et al., A 3D map of the human genome at kilobase resolution reveals principles of chromatin looping. Cell 159, 1665-1680 (2014).

71. H. K. Long, S. L. Prescott, J. Wysocka, Ever-changing landscapes: transcriptional enhancers in development and evolution. Cell 167, 1170-1187 (2016).

72. J. M. Alexander et al., Live-cell imaging reveals enhancer-dependent Sox 2 transcription in the absence of enhancer proximity. eLife 8, e41769 (2019).

73. S. Sainsbury, C. Bernecky, P. Cramer, Structural basis of transcription initiation by RNA polymerase II. Nat. Rev. Mol. Cell Biol. 16, 129-143 (2015).

74. H. Szutorisz, N. Dillon, L. Tora, The role of enhancers as centres for general transcription factor recruitment. Trends Biochem. Sci. 30, 593-599 (2005).

75. V. Haberle, A. Stark, Eukaryotic core promoters and the functional basis of transcription initiation. Nat. Rev. Mol. Cell Biol. 19, 621-637 (2018).

76. Y. Aoi et al., NELF regulates a promoter-proximal step distinct from RNA Pol II pause-release. Mol. Cell 78, 261-274 (2020).

77. S. Goutelle et al., The Hill equation: a review of its capabilities in pharmacological modelling. Fundam. Clin. Pharmacol. 22, 633-648 (2008).

78. N. S. Benabdallah et al., Decreased enhancer-promoter proximity accompanying enhancer activation. Mol. Cell 76, 473-484 (2019).

79. MATLAB and Statistics Toolbox Release 2021b, The MathWorks, Inc., Natick, Massachusetts, United States.

80. K. Noma, C. D. Allis, S. I. Grewal, Transitions in distinct histone H3 methylation patterns at the heterochromatin domain boundaries. Science 293, 1150-1155 (2001).

81. G. Thon, P. Bjerling, C. M. Bünner, J. Verhein-Hansen, Expression-state boundaries in the matingtype region of fission yeast. Genetics 161, 611-622 (2002).

82. L. Barinov, S. Ryabichko, W. Bialek, T. Gregor, Transcription-dependent spatial organization of a gene locus. arXiv preprint arXiv:2012.15819 (2020).

83. P. Thomas, N. Popović, R. Grima, Phenotypic switching in gene regulatory networks. Proc. Natl. Acad. Sci. U.S.A. 111, 6994-6999 (2014).

84. N. Friedman, L. Cai, X. S. Xie, Linking stochastic dynamics to population distribution: An analytical framework of gene expression. Phys. Rev. Lett. 97, 168302 (2006).

85. G. Nicoletti, D. M. Busiello, Mutual information disentangles interactions from changing environments. Phys. Rev. Lett. 127, 228301 (2021).

86. M. Bohn, D. W. Heermann, R. van Driel, Random loop model for long polymers. Phys. Rev. E 76, 051805 (2007).

87. C. W. Gardiner, Handbook of stochastic methods for physics, chemistry and the natural sciences. 
(Springer, Berlin, 1983).

88. K. S. Miller, On the inverse of the sum of matrices. Mathematics magazine 54, 67-72 (1981).

89. C. Da Fonseca, J. Petronilho, Explicit inverses of some tridiagonal matrices. Linear Algebra Appl. 325, 7-21 (2001).

90. J. S. Carroll et al., Chromosome-wide mapping of estrogen receptor binding reveals long-range regulation requiring the forkhead protein FoxA1. Cell 122, 33-43 (2005).

91. Y. F. Pan et al., Regulation of estrogen receptor-mediated long range transcription via evolutionarily conserved distal response elements. J. Biol. Chem. 283, 32977-32988 (2008).

92. D. A. Stavreva et al., Dynamics of chromatin accessibility and long-range interactions in response to glucocorticoid pulsing. Genome Res. 25, 845-857 (2015).

93. L. Vian et al., The energetics and physiological impact of cohesin extrusion. Cell 173, 1165-1178 (2018).

94. A. S. Hansen, E. K. O'Shea, Limits on information transduction through amplitude and frequency regulation of transcription factor activity. eLife 4, e06559 (2015).

95. M. R. Gartenberg, F. R. Neumann, T. Laroche, M. Blaszczyk, S. M. Gasser, Sir-mediated repression can occur independently of chromosomal and subnuclear contexts. Cell 119, 955-967 (2004).

96. B. Zoller, S. C. Little, T. Gregor, Diverse spatial expression patterns emerge from unified kinetics of transcriptional bursting. Cell 175, 835-847 (2018).

97. A. R. Krebs et al., Genome-wide single-molecule footprinting reveals high RNA polymerase II turnover at paused promoters. Mol. Cell 67, 411-422 (2017).

98. S. Bottani, R. A. Veitia, Hill function - based models of transcriptional switches: impact of specific, nonspecific, functional and nonfunctional binding. Biol. Rev. 92, 953-963 (2017).

99. T. O'Brien, J. T. Lis, Rapid changes in Drosophila transcription after an instantaneous heat shock. Mol. Cell. Biol. 13, 3456-3463 (1993).

100. A. W. Shermoen, P. H. O'Farrell, Progression of the cell cycle through mitosis leads to abortion of nascent transcripts. Cell 67, 303-310 (1991).

101. C. S. Thummel, K. C. Burtis, D. S. Hogness, Spatial and temporal patterns of E74 transcription during Drosophila development. Cell 61, 101-111 (1990).

102. J. Yao, M. B. Ardehali, C. J. Fecko, W. W. Webb, J. T. Lis, Intranuclear distribution and local dynamics of RNA polymerase II during transcription activation. Mol. Cell 28, 978-990 (2007).

103. M. D. Adams et al., The genome sequence of Drosophila melanogaster. Science 287, 2185-2195 (2000).

104. T. J. Richmond, C. A. Davey, The structure of DNA in the nucleosome core. Nature 423, 145-150 (2003).

105. E. Z. Kvon et al., Genome-scale functional characterization of Drosophila developmental enhancers in vivo. Nature 512, 91-95 (2014).

106. E. M. Blackwood, J. T. Kadonaga, Going the distance: a current view of enhancer action. Science 281, 60-63 (1998).

107. A. Erives, M. Levine, Coordinate enhancers share common organizational features in the Drosophila genome. Proc. Natl. Acad. Sci. U.S.A. 101, 3851-3856 (2004).

108. N. C. Grieder, T. Marty, H. D. Ryoo, R. S. Mann, M. Affolter, Synergistic activation of a Drosophila enhancer by HOM/EXD and DPP signaling. EMBO J. 16, 7402-7410 (1997).

109. R. A. Hoskins et al., Genome-wide analysis of promoter architecture in Drosophila melanogaster. 
Genome Res. 21, 182-192 (2011).

110. T. A. Down, C. M. Bergman, J. Su, T. J. Hubbard, Large-scale discovery of promoter motifs in Drosophila melanogaster. PLoS Comput. Biol. 3, e7 (2007).

111. H. Lodish et al., Molecular cell biology. (Scientific American Books, New York, 1995).

112. J. N. Weiss, The Hill equation revisited: uses and misuses. FASEB J. 11, 835-841 (1997).

113. D. I. Cattoni et al., Single-cell absolute contact probability detection reveals chromosomes are organized by multiple low-frequency yet specific interactions. Nat. Commun. 8, 1753 (2017).

114. A. Berrocal, N. C. Lammers, H. G. Garcia, M. B. Eisen, Kinetic sculpting of the seven stripes of the Drosophila even-skipped gene. eLife 9, e61635 (2020).

115. J. Falo-Sanjuan, N. C. Lammers, H. G. Garcia, S. J. Bray, Enhancer priming enables fast and sustained transcriptional responses to Notch signaling. Dev. Cell 50, 411-425 (2019).

116. R. Milo, R. Phillips, Cell biology by the numbers (Garland Science, 2015).

117. T. Fukaya, B. Lim, M. Levine, Rapid rates of Pol II elongation in the Drosophila embryo. Curr. Biol. 27, 1387-1391 (2017).

118. A. Senecal et al., Transcription factors modulate c-Fos transcriptional bursts. Cell Rep. 8, 75-83 (2014).

119. M. Yokoshi, K. Segawa, T. Fukaya, Visualizing the role of boundary elements in enhancerpromoter communication. Mol. Cell 78, 224-235 (2020).

120. J. Zuin et al., Nonlinear control of transcription through enhancer-promoter interactions. bioRxiv (2021).

121. M. C. Walters et al., Enhancers increase the probability but not the level of gene expression. Proc. Natl. Acad. Sci. U.S.A. 92, 7125-7129 (1995).

122. H. G. Sutherland, D. I. Martin, E. Whitelaw, A globin enhancer acts by increasing the proportion of erythrocytes expressing a linked transgene. Mol. Cell. Biol. 17, 1607-1614 (1997).

123. H. Ochiai et al., Genome-wide kinetic properties of transcriptional bursting in mouse embryonic stem cells. Sci. Adv. 6, eaaz6699 (2020). 